TRANSACTIONS OF THE

AMERICAN MATHEMATICAL SOCIETY

Volume 366, Number 1, January 2014, Pages 341-393

S 0002-9947(2013)05837-0

Article electronically published on June 10, 2013

\title{
DEHN FILLINGS OF KNOT MANIFOLDS CONTAINING ESSENTIAL ONCE-PUNCTURED TORI
}

\author{
STEVEN BOYER, CAMERON McA. GORDON, AND XINGRU ZHANG
}

\begin{abstract}
In this paper we study exceptional Dehn fillings on hyperbolic knot manifolds which contain an essential once-punctured torus. Let $M$ be such a knot manifold and let $\beta$ be the boundary slope of such an essential once-punctured torus. We prove that if Dehn filling $M$ with slope $\alpha$ produces a Seifert fibred manifold, then $\Delta(\alpha, \beta) \leq 5$. Furthermore we classify the triples $(M ; \alpha, \beta)$ when $\Delta(\alpha, \beta) \geq 4$. More precisely, when $\Delta(\alpha, \beta)=5$, then $M$ is the (unique) manifold $W h(-3 / 2)$ obtained by Dehn filling one boundary component of the Whitehead link exterior with slope $-3 / 2$, and $(\alpha, \beta)$ is the pair of slopes $(-5,0)$. Further, $\Delta(\alpha, \beta)=4$ if and only if $(M ; \alpha, \beta)$ is the triple $\left(W h\left(\frac{-2 n \pm 1}{n}\right) ;-4,0\right)$ for some integer $n$ with $|n|>1$. Combining this with known results, we classify all hyperbolic knot manifolds $M$ and pairs of slopes $(\beta, \gamma)$ on $\partial M$ where $\beta$ is the boundary slope of an essential once-punctured torus in $M$ and $\gamma$ is an exceptional filling slope of distance 4 or more from $\beta$. Refined results in the special case of hyperbolic genus one knot exteriors in $S^{3}$ are also given.
\end{abstract}

\section{INTRODUCTION}

This is the second of four papers in which we investigate the following conjecture of the second-named author (see Go2, Conjecture 3.4]). Recall that a hyperbolic knot manifold is a compact, connected, orientable 3-manifold with torus boundary whose interior admits a complete, finite volume hyperbolic structure.

Conjecture 1.1 (C. McA. Gordon). Suppose that $M$ is a hyperbolic knot manifold and $\alpha, \beta$ are slopes on $\partial M$ such that $M(\alpha)$ is Seifert fibred and $M(\beta)$ is toroidal. If $\Delta(\alpha, \beta)>5$, then $M$ is the figure eight knot exterior.

Our first result reduces the verification of the conjecture to the case where the Seifert filling is atoroidal.

Theorem 1.2. Suppose that $M$ is a hyperbolic knot manifold and $\alpha, \beta$ are slopes on $\partial M$ such that $M(\alpha)$ is a toroidal Seifert fibred manifold and $M(\beta)$ is toroidal. Then $\Delta(\alpha, \beta) \leq 4$. Furthermore, if $\Delta(\alpha, \beta)=4$, then $(M ; \alpha, \beta) \cong\left(N\left(-\frac{1}{2},-\frac{1}{2}\right) ;-4,0\right)$, where $N$ is the exterior of the 3 -chain link $\mathrm{MP}$.

We have that $N\left(-\frac{1}{2},-\frac{1}{2},-4\right)$ is Seifert fibred with base orbifold $P^{2}(2,3)$ and $N\left(-\frac{1}{2},-\frac{1}{2}, 0\right)$ contains an incompressible torus separating $N\left(-\frac{1}{2},-\frac{1}{2}, 0\right)$ into Seifert fibred manifolds with base orbifolds $D^{2}(2,2)$ and $D^{2}(2,3)$. (See [MP Table 2].)

Received by the editors November 8, 2011 and, in revised form, March 23, 2012.

2010 Mathematics Subject Classification. Primary 57M25, 57M50, 57M99.

The first author was partially supported by NSERC grant RGPIN 9446-2008.

The second author was partially supported by NSF grant DMS-0906276. 
A small Seifert manifold is a 3-manifold which admits a Seifert structure with base orbifold of the form $S^{2}(a, b, c)$, where $a, b, c \geq 1$. For instance, a closed, atoroidal Seifert manifold is small Seifert.

A small Seifert manifold is a prism manifold if its base orbifold is $S^{2}(2,2, n)$ for some $n \geq 2$.

Since the distance between a toroidal filling slope and a reducible filling slope is at most 3 ([Oh], Wu1 $)$, Theorem 1.2 reduces our analysis of Conjecture 1.1 to understanding the case where the Seifert Dehn filling is irreducible and small Seifert. In an earlier paper BGZ2 we verified the conjecture in the case where $M$ admits no essential punctured torus of boundary slope $\beta$ which is a fibre or semi-fibre, or which has fewer than three boundary components; more precisely, we showed that in this case $\Delta(\alpha, \beta) \leq 5$. Here we focus on the case where $M$ admits an essential punctured torus with one boundary component.

Let $W h$ denote the left-handed Whitehead link exterior (see Figure 33). We parameterise the slopes on a boundary component of $W h$ using the standard meridianlongitude coordinates.

Theorem 1.3. Let $M$ be a hyperbolic knot manifold and $\alpha$ a slope on $\partial M$ such that $M(\alpha)$ is small Seifert. If $M$ admits an essential, once-punctured torus $F$ of boundary slope $\beta$, then $\Delta(\alpha, \beta) \leq 5$. Further, if $\Delta(\alpha, \beta)>3$, then $F$ is not a fibre and $\pi_{1}(M(\alpha))$ is finite. More precisely,

(1) if $\Delta(\alpha, \beta)=4$, then $(M ; \alpha, \beta) \cong\left(W h\left(\frac{-2 n \pm 1}{n}\right) ;-4,0\right)$ for some integer $n$ with $|n|>1$ and $M(\alpha)$ has base orbifold $S^{2}(2,2,|\mp 2 n-1|)$, so $M(\alpha)$ is a prism manifold;

(2) if $\Delta(\alpha, \beta)=5$, then $(M ; \alpha, \beta) \cong(W h(-3 / 2) ;-5,0)$, and $M(\alpha)$ has base orbifold $S^{2}(2,3,3)$.

Baker $\mathrm{Ba}$ ] has proven Theorem 1.3 in the case where $M(\alpha)$ is a lens space. We provide an alternate proof of his result.

Theorem 1.3 is sharp; see the infinite family of examples in $\$ 11$ for (1) and [MP, Table A.3] for (2). Another family of examples is provided by hyperbolic twist knots. These are genus one knots in the 3 -sphere whose exteriors admit small Seifert filling slopes of distance 1,2, and 3 from the longitudinal slope. Finally, Baker Ba, Theorem 1.1(IV)] has constructed an infinite family of non-fibred hyperbolic knot manifolds which admit a once-punctured essential torus whose boundary slope is of distance 3 to a lens space filling slope.

Here is an outline of the proof of Theorem 1.3 We begin by showing that the result holds unless, perhaps, $M$ admits an orientation-preserving involution $\tau$ with non-empty branch set $L$ contained in the interior of the quotient $M / \tau$, which is a solid torus. The results of BGZ2 reduce us to the case that $L$ has a very particular form (see Figure 3). On the other hand, $\tau$ extends to an involution $\tau_{\alpha}$ of $M(\alpha)$ with branch set $L_{\alpha}$ contained in the lens space $M(\alpha) / \tau_{\alpha}$. The fundamental group of $M(\alpha) / \tau_{\alpha}$ is non-trivial if the distance between $\alpha$ and $\beta$ is at least 3. Since the involutions on small Seifert manifolds with such quotients are well understood, we can explicitly describe the branch set $L_{\alpha}$ of $\tau_{\alpha}$. Comparing this description with the constraints we have already deduced on $L$ leads to the proof of the theorem.

Recall that an exceptional filling slope on the boundary of a hyperbolic 3-manifold is a slope $\gamma$ such that $M(\gamma)$ is not hyperbolic. Geometrisation of 3-manifolds implies that a slope $\gamma$ is exceptional if and only if $M(\gamma)$ is either reducible, toroidal, 
or Seifert fibred. Theorem 1.3 combines with [Oh], [Wu1, Go1, GW], and Proposition 3.1 to yield the next result.

Theorem 1.4. Let $M$ be a hyperbolic knot manifold which admits an essential, once-punctured torus $F$ of boundary slope $\beta$ and let $\gamma$ be an exceptional filling slope on $\partial M$.

(1) $\Delta(\gamma, \beta) \leq 7$.

(2) If $\Delta(\gamma, \beta)>3$, then $M(\gamma)$ is either toroidal or has a finite fundamental group.

(3) If $\Delta(\gamma, \beta)>3$ and $M(\gamma)$ is toroidal, then either

(a) $\Delta(\gamma, \beta)=4$ and $(M ; \gamma, \beta) \cong(W h(\delta) ;-4,0)$ for some slope $\delta$, or

(b) $\Delta(\gamma, \beta)=5$ and $(M ; \gamma, \beta) \cong(W h(-4 / 3) ;-5,0)$ or $(W h(-7 / 2) ;-5 / 2,0)$, or

(c) $\Delta(\gamma, \beta)=7$ and $(M ; \gamma, \beta) \cong(W h(-5 / 2) ;-7 / 2,0)$.

(4) If $\Delta(\gamma, \beta)>3$ and $\pi_{1}(M(\gamma))$ is finite, then either

(a) $\Delta(\gamma, \beta)=4,(M ; \gamma, \beta) \cong\left(W h\left(\frac{-2 n \pm 1}{n}\right) ;-4,0\right)$ for some integer $n$ with $|n|>1$, and $M(\gamma)$ has base orbifold $S^{2}(2,2,|\mp 2 n-1|)$, or

(b) $\Delta(\gamma, \beta)=5,(M ; \gamma, \beta) \cong(W h(-3 / 2) ;-5,0)$, and $M(\gamma)$ has base orbifold $S^{2}(2,3,3)$.

Next we specialize to the case where $M$ is the exterior of a hyperbolic knot in the 3 -sphere.

Theorem 1.5. Let $K \subset S^{3}$ be a hyperbolic knot of genus one with exterior $M_{K}$ and suppose $p / q$ is an exceptional filling slope on $\partial M_{K}$.

(1) $M_{K}(0)$ is toroidal but not Seifert.

(2) $M_{K}(p / q)$ is either toroidal or small Seifert with hyperbolic base orbifold.

(3) If $M_{K}(p / q)$ is small Seifert with hyperbolic base orbifold, then $0<|p| \leq 3$.

(4) If $M_{K}(p / q)$ is toroidal, then $|q|=1$ and $|p| \leq 4$ with equality implying $K$ is a twist knot.

Here is how the paper is organised. We prove Theorem 1.2 in $\$ 2$ In $\$ 3$ we show that there are strong topological constraints on $M$ which must be satisfied if Theorem 1.3 doesn't hold. These constraints will be applied later in the paper to construct an involution on $M$. In $\$ 4$ we describe the branching set of an orientation-preserving involution on a small Seifert manifold with quotient space a lens space with non-trivial fundamental group. Using this, in $\$ 5$ we reduce the proof of Theorem 1.3 to five problems involving links in lens spaces and a problem in which $\Delta(\alpha, \beta)=4$ and $M(\alpha)$ is a prism manifold. These problems are resolved in $\$ 6,87,88,99, \$ 10$ and $\$ 12$, respectively. The infinite family of examples realising distance 4 in Theorem 1.3 is constructed in $\$ 11$ Theorems 1.4 and 1.5 are dealt with in $\$ 13$.

\section{The CASE Where $M(\alpha)$ IS TOROIDAL}

In this section we prove Theorem 1.2. Recall from the introduction that $N$ denotes the exterior of the 3 -chain link of [MP]. Note that $N\left(-\frac{1}{2},-\frac{1}{2}\right)$ is obtained by Dehn filling on $N\left(-\frac{1}{2}\right)$, which is the exterior of the rational link associated with the rational number $10 / 3$.

To prove Theorem 1.2 we consider all $(M ; \alpha, \beta)$ where $M$ is hyperbolic, $M(\alpha)$ and $M(\beta)$ are toroidal and $\Delta(\alpha, \beta) \geq 4$. For $\Delta(\alpha, \beta) \geq 6$ there are only four such $(M ; \alpha, \beta)$ Go1, and in all four cases neither $M(\alpha)$ nor $M(\beta)$ is Seifert fibred. 
For $\Delta(\alpha, \beta)=4$ or 5 , the triples $(M ; \alpha, \beta)$ are determined in $[\mathrm{GW}$ : there are 14 hyperbolic manifolds $M_{i}, 1 \leq i \leq 14$, each with a pair of toroidal filling slopes $\alpha_{i}, \beta_{i}$ at distance 4 or 5 , where $M_{1}, M_{2}, M_{3}$ and $M_{14}$ have two (torus) boundary components, and the others, one. It is shown in GW that a hyperbolic manifold $M$ has two toroidal filling slopes $\alpha$ and $\beta$ at distance 4 or 5 if and only if $(M ; \alpha, \beta) \cong$ $\left(M_{i} ; \alpha_{i}, \beta_{i}\right)$ for some $1 \leq i \leq 14$, or $(M ; \alpha, \beta) \cong\left(M_{i}(\gamma) ; \alpha_{i}, \beta_{i}\right)$ for $i=1,2,3$ or 14 and some slope $\gamma$ on the second boundary component of $M_{i}$. (We adopt the convention that in the above homeomorphisms either $\alpha \mapsto \alpha_{i}, \beta \mapsto \beta_{i}$ or $\alpha \mapsto \beta_{i}$, $\beta \mapsto \alpha_{i}$.) We prove Theorem 1.2 by showing first, for $i \neq 1,2,3$ or 14 , neither of the toroidal manifolds $M_{i}\left(\alpha_{i}\right)$ or $M_{i}\left(\beta_{i}\right)$ is Seifert fibred, second, for $i=1,3$ or 14 , there is no hyperbolic manifold of the form $M_{i}(\gamma)$ with either $M_{i}(\gamma)\left(\alpha_{i}\right)$ or $M_{i}(\gamma)\left(\beta_{i}\right)$ toroidal Seifert fibred and third, there is a unique example $\left(M_{2}(\gamma) ; \alpha_{2}, \beta_{2}\right)$ (up to homeomorphism) where $M_{2}(\gamma)$ is hyperbolic, $M_{2}(\gamma)\left(\alpha_{2}\right)$ and $M_{2}(\gamma)\left(\beta_{2}\right)$ are toroidal, and one is Seifert fibred; this is the example described in Theorem 1.2 .

We first consider the manifolds $M_{i}, 6 \leq i \leq 13$. The toroidal fillings on $M_{i}$, $M_{i}(0)$ and $M_{i}\left(\beta_{i}\right)$, are described in Lemma 22.2 of [GW]. We adopt the notation introduced in [GW, page 116].

Lemma 2.1. For $6 \leq i \leq 13, M_{i}(0)$ is not Seifert fibred.

Proof. $M_{i}(0)$ is of the form $X\left(p_{1}, q_{1} ; p_{2}, q_{2}\right)$; it is the double branched cover of the tangle $Q_{i}(0)$, which is of the form $T\left(p_{1}, q_{1} ; p_{2}, q_{2}\right)$, the union of two Montesinos tangles. Assume the numbering is chosen so that $p_{1}, q_{1}$ are not both 2 (actually this is only an issue when $i=8)$. Then the Seifert fibre $\varphi_{1}$ of $X\left(p_{1}, q_{1}\right)$ is unique. Since $X\left(p_{1}, q_{1}\right)$ and $X\left(p_{2}, q_{2}\right)$ are not both twisted $I$-bundles, to show that $M_{i}(0)$ is not Seifert fibred it suffices to show that, in the gluing of $X\left(p_{1}, q_{1}\right)$ and $X\left(p_{2}, q_{2}\right), \varphi_{1}$ is not identified with the Seifert fibre $\varphi_{2}$ of $X\left(p_{2}, q_{2}\right)$. (When $i=8, p_{2}=q_{2}=2$ and there are two possible choices for $\varphi_{2}$.) We do this by identifying the image of $\varphi_{1}$ in the boundary of the tangle $T\left(p_{1}, q_{1}\right)$ and then capping off the tangle $T\left(p_{2}, q_{2}\right)$ with the corresponding rational tangle. In the double branched cover this corresponds to doing Dehn filling on $X\left(p_{2}, q_{2}\right)$ along the slope $\varphi_{1}$. If $M_{i}(0)$ were Seifert fibred, then this Dehn filling would be reducible, and so the corresponding rational tangle filling on $T\left(p_{2}, q_{2}\right)$ would give a link that is either composite or split. One checks that this is not the case.

Lemma 2.2. For $6 \leq i \leq 13, M_{i}\left(\beta_{i}\right)$ is not Seifert fibred.

Proof. First note that $M_{7}\left(\beta_{7}\right)$ is of the form $X(2,3 ; 2,2)$. We check that this is not Seifert fibred in the same way as we did for $M_{8}(0)$ in Lemma 2.1

When $i \neq 7, M_{i}\left(\beta_{i}\right)$ is the double branched cover of a 2-component link $L_{i}$; see GW, Lemma 22.2]. More specifically, for $i=6,8,9$ or $12, L_{i}$ is a cabled Hopf link $C\left(p_{1}, q_{1} ; p_{2}, q_{2}\right)$ with $p_{1}, p_{2}>1$, for $i=10$ or $11, L_{i}$ is the link $C(C ; 2,1)$ (see GW, page 116]), and for $i=13, L_{i}$ is the 2-string cable of the trefoil shown in [GW] Figure 22.13(d)]. In all cases, $L_{i}$ is toroidal, i.e. its exterior contains an essential torus. Moreover, the exterior of $L_{i}$ is not Seifert fibred. Therefore if $M_{i}\left(\beta_{i}\right)$ were Seifert fibred, then $L_{i}$ would be a Montesinos link. But the only toroidal Montesinos links are (see [Oe, Corollary 5]) $K\left(\frac{1}{2}, \frac{1}{2},-\frac{1}{2},-\frac{1}{2}\right), K\left(\frac{2}{3},-\frac{1}{3},-\frac{1}{3}\right), K\left(\frac{1}{2},-\frac{1}{4},-\frac{1}{4}\right)$, and $K\left(\frac{1}{2},-\frac{1}{3},-\frac{1}{6}\right)$. One easily checks that no $L_{i}$ is of this form.

Lemma 2.3. $M_{4}\left(\alpha_{4}\right)$ and $M_{4}\left(\beta_{4}\right)$ are not Seifert fibred. 
Proof. $M_{4}\left(\alpha_{4}\right)$ and $M_{4}\left(\beta_{4}\right)$ contain incompressible tori $\widehat{F}_{a}$ and $\widehat{F}_{b}$; the corresponding punctured tori $F_{a}$ and $F_{b}$ in $M_{4}$ have four and two boundary components, respectively. The intersection of $F_{a}$ and $F_{b}$ is described by the intersection graphs $\Gamma_{a} \subset \widehat{F}_{a}$ and $\Gamma_{b} \subset \widehat{F}_{b}$ depicted in Figures 11.9(a) and (b) of [GW, respectively. Note that $\widehat{F}_{a}$ separates $M_{4}\left(\alpha_{4}\right)$, into $M_{B}$ and $M_{W}$, say, while $\widehat{F}_{b}$ is non-separating in $M_{4}\left(\beta_{4}\right)$. The faces of the graph $\Gamma_{b}$ lie alternately in $M_{B}$ and $M_{W}$; we choose the notation so that all the faces of $\Gamma_{b}$ that lie in $M_{B}$ are bigons.

Let $f_{1}, f_{2}, f_{3}$, and $g_{1}, g_{2}, g_{3}$ be the faces of $\Gamma_{b}$ with edges $G, H ; J, K ; A, B$; and $D, E ; K, P, R ; A, G, L$; respectively. Let $h_{1}, h_{2}, h_{3}$ be the faces of $\Gamma_{a}$ with edges $E, N ; H, E$; and $B, G, N, R$; respectively. (The notation refers to the edges illustrated in Figure 11.9 of $[\mathrm{GW}]$.)

For computations in $\pi_{1}\left(M_{B}\right)$ and $\pi_{1}\left(M_{W}\right)$ we take as "base-point" the rectangle in $\widehat{F}_{a}$ shown in Figure 11.9(a) of [GW]. Let $s, t$ be the pair of generators of $\pi_{1}\left(\widehat{F}_{a}\right)$ determined by the downward vertical and rightward horizontal edges of that rectangle, respectively. Let $x_{1}$ and $x_{3}$ be the elements of $\pi_{1}\left(M_{B}\right)$ corresponding to the 1-handles $H_{(12)}$ and $H_{(34)}$ in the usual way. The faces $f_{1}, f_{2}$ and $f_{3}$ give the relations in $\pi_{1}\left(M_{B}\right)$ :

$$
\begin{aligned}
& x_{1}^{2} t=1, \\
& x_{3}^{2} t^{-1}=1, \\
& s^{-1} x_{3} x_{1}=1 .
\end{aligned}
$$

It follows that $M_{B}$ is Seifert fibred with base orbifold $D^{2}(2,2)$ and that the classes in $\pi_{1}\left(\widehat{F}_{a}\right)$ of the Seifert fibres in the two Seifert fibrings of $M_{B}$ are $t$ and $s$.

Let $x_{2}$ and $x_{4}$ be the elements of $\pi_{1}\left(M_{W}\right)$ corresponding to $H_{(23)}$ and $H_{(41)}$. Then the faces $g_{1}, g_{2}$ and $g_{3}$ give the relations in $\pi_{1}\left(M_{W}\right)$ :

$$
\begin{aligned}
& t x_{4} x_{2}=1, \\
& x_{2} x_{4} t^{-1} x_{2} s t=1, \\
& x_{2} x_{4}^{2} t^{-1}=1 .
\end{aligned}
$$

These show that $M_{W}$ is Seifert fibred with base orbifold $D^{2}(2,3)$, the class of the Seifert fibre in $\pi_{1}\left(\widehat{F}_{a}\right)$ being $s t^{2}$. Since this is distinct from either of the Seifert fibres of $M_{B}, M_{4}\left(\alpha_{4}\right)$ is not Seifert fibred.

We now consider $M_{4}\left(\beta_{4}\right)$. Let $u, v$ be the pair of generators for $\pi_{1}\left(\widehat{F}_{b}\right)$ given by the downward vertical and leftward horizontal edges of the rectangle in Figure 11.9 (b) of [GW]. (We take this rectangle as "base-point" for computations in $\pi_{1}\left(M_{4}\left(\beta_{4}\right)\right)$.) Let $x, y$ be the elements of $\pi_{1}\left(M_{4}\left(\beta_{4}\right)\right)$ given by the 1-handles $H_{(12)}$ and $H_{(21)}$. The faces $h_{1}, h_{2}, h_{3}$ give the relations in $\pi_{1}\left(M_{4}\left(\beta_{4}\right)\right)$ :

$$
\begin{aligned}
& x(u v) y^{-1} v^{-1}=1, \\
& y v x^{-1}=1, \\
& x^{-1} u^{-1} x u x^{-1}(v u)^{-1} y=1 .
\end{aligned}
$$

The second relation gives $x=y v$, and the first then gives

$$
y^{-1} v y=u v^{2} \text {. }
$$

The third relation gives

$$
\left(y^{-1} u^{-1} y\right) u\left(y^{-1} u^{-1} y\right) u^{-1} v^{-3}=1 .
$$


Now if $M_{4}\left(\beta_{4}\right)$ were Seifert fibred, the non-separating torus $\widehat{F}_{b}$ would be horizontal, and so $M_{4}\left(\beta_{4}\right)$ would be a torus bundle over the circle with fibre $\widehat{F}_{b}$. Hence $y^{-1} u^{-1} y$ would belong to $\pi_{1}\left(\widehat{F}_{b}\right)$. But the last relation above shows that if this is the case, then

$$
\left(y^{-1} u^{-1} y\right)^{2}=v^{3} .
$$

Since $v^{3}$ is not a square in $\pi_{1}\left(\widehat{F}_{b}\right)$, this is a contradiction.

Lemma 2.4. $M_{5}\left(\alpha_{5}\right)$ and $M_{5}\left(\beta_{5}\right)$ are not Seifert fibred.

Proof. This can be proved in a similar fashion to Lemma 2.3, using GW, Figure 11.10]. Another way to establish the result is to note that, according to [L2, $\S 6], M_{5} \cong N\left(1,-\frac{1}{3}\right)$, the toroidal filling slopes $\alpha_{5}, \beta_{5}$ being -4 and 1 . We see that $N\left(1,-\frac{1}{3},-4\right)$ and $N\left(1,-\frac{1}{3}, 1\right)$ are not Seifert fibred from Tables 4 and 3 of [MP], respectively.

We next consider the manifolds $M_{1}, M_{2}$ and $M_{3}$, namely the exteriors of the Whitehead link, the 10/3-rational link, and the Whitehead sister (or $(-2,3,8)$ pretzel) link, respectively. These are all obtained by Dehn filling on the 3 -chain link: $M_{1} \cong N(1), M_{2} \cong N\left(-\frac{1}{2}\right), M_{3} \cong N(-4)$. Furthermore, their exceptional slopes and toroidal slopes are as follows (see [MP, Table A.1]):

\begin{tabular}{|c|l|c|}
\hline & exceptional slopes & toroidal slopes \\
\hline$N(1)$ & $\infty,-3,-2,-1,0,1$ & $-3,1$ \\
\hline$N\left(-\frac{1}{2}\right)$ & $\infty,-4,-3,-2,-1,0$ & $-4,0$ \\
\hline$N(-4)$ & $\infty,-3,-2,-1,-\frac{1}{2}, 0$ & $-\frac{1}{2}, 0$ \\
\hline
\end{tabular}

Lemma 2.5. In each of the following cases, the manifold $N(\alpha, \beta, \gamma)$ is a toroidal Seifert fibre space if and only if $\gamma$ is one of the values listed:

$$
\begin{array}{lll}
\text { (a) } & N(1,-3, \gamma): & \gamma=-3,1, \\
& N(1,1, \gamma): & \gamma=-3,-2,-1,0 . \\
\text { (b) } & N\left(-\frac{1}{2},-4, \gamma\right): & \gamma=-\frac{1}{2}, \\
& N\left(-\frac{1}{2}, 0, \gamma\right): & \gamma=-\frac{7}{2} . \\
\text { (c) } & N\left(-4,-\frac{1}{2}, \gamma\right): & \gamma=-\frac{1}{2}, \\
& N(-4,0, \gamma): & \text { no } \gamma .
\end{array}
$$

Proof. This follows by inspecting Tables 2, 3 and 4 of [MP]. We see from these that the only toroidal Seifert fibre spaces $N(\alpha, \beta, \gamma)$ are

(1) $N(-3,1,1), N\left(-3,-\frac{5}{3},-\frac{5}{3}\right), N(-3,-3, t / u)$ where $t / u \neq-1,-1+\frac{1}{m}$ or $\infty$, and

(2) $N\left(0, \frac{1}{2}+n,-\frac{9}{2}-n\right), N(1,1, n)$ where $|n+1| \leq 1, N\left(-\frac{3}{2},-\frac{5}{2}, 0\right)$, and $N\left(-4,-\frac{1}{2},-\frac{1}{2}\right)$.

Note that the values of $\gamma$ listed in parts (a) and (c) of Lemma 2.5] all belong to the set of exceptional slopes of $N(1)$ and $N(-4)$, respectively. It follows that for $i=1$ and 3 , there is no $\gamma$ such that $M_{i}(\gamma)$ is hyperbolic and one of $M_{i}(\gamma)\left(\alpha_{i}\right)$, $M_{i}(\gamma)\left(\beta_{i}\right)$ is toroidal Seifert fibred. 
In the case $i=2$, note that by $[\mathrm{MP}$, Proposition 1.5 part (1.4)], there is an automorphism of $N\left(-\frac{1}{2}\right)$ inducing homeomorphisms

$$
\begin{aligned}
& N\left(-\frac{1}{2},-4,-\frac{1}{2}\right) \cong N\left(-\frac{1}{2}, 0,-\frac{7}{2}\right), \\
& N\left(-\frac{1}{2}, 0,-\frac{1}{2}\right) \cong N\left(-\frac{1}{2},-4,-\frac{7}{2}\right) .
\end{aligned}
$$

Also, we see from [MP, Table 2] that $N\left(-\frac{1}{2}, 0,-\frac{1}{2}\right)$ is toroidal. Thus part (b) of Lemma 2.5 gives rise to the single example described in Theorem 1.2 .

Finally, we take care of $M_{14}$ :

Lemma 2.6. For no slope $\gamma$ on the second boundary component of $M_{14}$ is $M_{14}(\gamma)\left(\alpha_{14}\right)$ or $M_{14}(\gamma)\left(\beta_{14}\right)$ toroidal Seifert fibred.

Proof. In [L1] Lee describes a hyperbolic 3-manifold $Y$ with two torus boundary components having (homeomorphic) Dehn fillings $Y(0)$ and $Y(4)$ that contain Klein bottles. In fact $Y(0) \cong Y(4) \cong Q(2,2) \cup W h$, where $Q(2,2)$ is the Seifert fibre space with base orbifold $D^{2}(2,2)$ and $W h$ is the exterior of the Whitehead link. Hence $Y(0) \cong Y(4)$ is toroidal. It follows from the classification in $\mathrm{GW}$ of the hyperbolic 3 -manifolds with toroidal fillings at distance 4 that $Y \cong M_{14}$. (The only other manifolds with two boundary components having toroidal fillings at distance 4 are $M_{1}$ and $M_{2}$, and there the toroidal fillings are graph manifolds; see e.g. [MP, Table A.1].) It therefore suffices to show that $M_{14}(\gamma)\left(\alpha_{14}\right)$ is not toroidal Seifert fibred for any slope $\gamma$.

The manifold $M=M_{14}\left(\alpha_{14}\right) \cong Q(2,2) \cup W h$ is the double branched cover of the tangle shown in GW, Figure 22.14(b)]. Thus $M(\gamma) \cong Q(2,2) \cup W h(\gamma)$. Hence if $M(\gamma)$ is toroidal Seifert fibred then $\gamma$ must be an exceptional slope for $W h$. These slopes (with respect to the parametrization in [MP, Table A.1]) are $\infty,-3,-2,-1,0$ and 1. Now $W h(-3)$ and $W h(1)$ are toroidal non-Seifert, $W h(\infty) \cong D^{2} \times S^{1}$, and $W h(-2), W h(-1)$ and $W h(0)$ are Seifert fibred with base orbifold $D^{2}(3,3), D^{2}(2,4)$ and $D^{2}(2,3)$, respectively. So we need only consider $M(\gamma)$ for $\gamma=\infty,-2,-1$ and 0 ; we do this by examining the corresponding rational tangle filling on the tangle shown in GW, Figure 22.14(b)]. For $\gamma=\infty$, this yields the pretzel knot $K\left(-\frac{1}{2},-\frac{1}{2}, \frac{1}{2}\right)$, so $M(\infty)$ is atoroidal. For $\gamma=-2,-1$ and 0 we show that the Seifert fibre of $W h(\gamma)$ does not match the Seifert fibre in either of the two Seifert fibrings of $Q(2,2)$. This is straightforward to check, for example by using the same approach as in the proof of Lemma 2.1.

\section{BACKGround Results For the PROOF of TheOREM 1.3}

We collect various results in this section and the next which will be used throughout this paper and its sequel BGZ3. In what follows, $M$ will be a hyperbolic knot manifold and $b_{1}(M)$ will denote its first Betti number. In this section we assume that $F$ is an essential, punctured torus of slope $\beta$ which is properly embedded in $M$.

For a closed, essential surface $S$ in $M$ we define $\mathcal{C}(S)$ to be the set of slopes $\delta$ on $\partial M$ such that $S$ compresses in $M(\delta)$. A slope $\eta$ on $\partial M$ is called a singular slope for $S$ if $\eta \in \mathcal{C}(S)$ and $\Delta(\delta, \eta) \leq 1$ for each $\delta \in \mathcal{C}(S)$. A result of Wu Wu2] states that if $\mathcal{C}(S) \neq \emptyset$, then there is at least one singular slope for $S$.

Proposition 3.1. Suppose that $M$ admits a non-separating, essential, genus 1 surface of boundary slope $\beta$ which caps-off to a compressible torus in $M(\beta)$. If $\gamma$ is 
a slope on $\partial M$ such that $M(\gamma)$ is not hyperbolic, then $\Delta(\gamma, \beta) \leq 3$. If $M(\gamma)$ is an irreducible, atoroidal, small Seifert manifold, then $\Delta(\gamma, \beta) \leq 1$.

Proof. By hypothesis $M(\beta)$ admits a non-separating 2-sphere and so is reducible with first Betti number at least 1 . In the case that $b_{1}(M) \geq 2$, there is a closed essential surface $S \subset \operatorname{int}(M)$ which is Thurston norm minimizing in $H_{2}(M)$. By Ga, Corollary], $S$ is essential and Thurston norm minimizing in $H_{2}(M(\delta))$ for all slopes $\delta \neq \beta$. By [BGZ1, Proposition 5.1], $\Delta(\gamma, \beta) \leq 1$ for any slope $\gamma$ such that $M(\gamma)$ is not hyperbolic. Suppose then that $b_{1}(M)=1$, and note that by hypothesis $\beta$ is a strict boundary slope. In this case [BCSZ2, Theorem 3.2] implies that $\beta$ is a singular slope, and so the conclusions of the lemma follow from BGZ1, Theorem $1.5]$.

Corollary 3.2. Theorem 1.3 holds if $M$ admits a non-separating, essential, genus 1 surface of boundary slope $\beta$ which caps-off to a compressible torus in $M(\beta)$.

The torus in $M(\beta)$ obtained by capping-off $F$ with a meridional disk will be denoted $\widehat{F}$. We use $M_{F}$ to denote the compact manifold obtained by cutting $M$ open along $F$ and $M(\beta)_{\widehat{F}}$ the manifold obtained by cutting $M(\beta)$ open along $\widehat{F}$.

Proposition 3.3. Suppose that $M(\alpha)$ is a Seifert fibred manifold and $M(\beta)$ is toroidal. Then $\Delta(\alpha, \beta) \leq 3$ as long as one of the following conditions is satisfied:

(a) $\alpha$ or $\beta$ is a singular slope of a closed essential surface in $M$.

(b) $M(\alpha)$ or $M(\beta)$ is reducible.

(c) (i) $|\partial F|=1$ and $M_{F}$ is not a genus 2 handlebody.

(ii) $|\partial F|=2$ and $M_{F}$ is neither connected nor a union of two genus 2 handlebodies.

Proof. If $\alpha$ or $\beta$ is a singular slope of a closed essential surface in $M$, then BGZ1, Corollary 1.6] shows that $\Delta(\alpha, \beta) \leq 3$, so we are done in case (a).

Assume next that $M(\gamma)$ is reducible, where $\gamma$ is one of $\alpha$ or $\beta$. If $\gamma=\alpha$, then $\Delta(\alpha, \beta) \leq 3$ by $\mathrm{Oh}$ and Wu1. Assume then that $\gamma=\beta$. If $b_{1}(M) \geq 2$, then $\Delta(\gamma, \beta) \leq 1$ for any exceptional slope $\gamma$ as in the proof of Proposition 3.1. Assume then that $b_{1}(M)=1$. Since $M(\beta)$ is toroidal, it is neither $S^{1} \times S^{2}$ nor a connected sum of lens spaces. Hence [BGZ1, Proposition 6.2] implies that $\beta$ is a singular slope of a closed essential surface in $M$. Thus we are done by part (a).

Finally consider part (c) of the proposition. If $|\partial F|=1$, any compression of $\partial M_{F}$ in $M_{F}$ yields one or two tori, so as $M$ is hyperbolic it is not hard to see that $M_{F}$ is a handlebody, contrary to hypothesis. Thus $\partial M_{F}$ is incompressible in $M_{F}$, and hence in $M$. Let $S \subset \operatorname{int}(M)$ be the inner boundary component of a collar of $\partial M_{F}$ in $M_{F}$. Then $S$ is incompressible in $M$, and by construction there is an annulus $A$ in $M$ with boundary components $\partial_{1} A$ and $\partial_{2} A$, say, where $A \cap S=\partial_{1} A$ and $A \cap \partial M=\partial_{2} A$ has slope $\beta$ on $\partial M$. It follows from Sh that $S$ is incompressible in $M(\gamma)$ whenever $\Delta(\gamma, \beta)>1$. Thus $\beta$ is a singular slope for $S$, and so part (a) of this proposition shows $\Delta(\alpha, \beta) \leq 3$. Thus (i) holds.

If $|\partial F|=2$ and $M_{F}$ is not connected, then $M=X_{1} \cup_{F} X_{2}$ where $\partial X_{j}$ is a genus 2 surface for $j=1,2$. If $\partial X_{j}$ compresses in $X_{j}$ for both $j$, then $X_{1}$ and $X_{2}$ are genus 2 handlebodies as $M$ is hyperbolic. Since this possibility is excluded by our hypotheses, $\partial X_{j}$ is incompressible in $X_{j}$ for some $j$. Then it is essential in $M$ but compresses in $M(\beta)$, so as in the previous paragraph, $\beta$ is a singular slope for $\partial X_{j}$. Thus $\Delta(\alpha, \beta) \leq 3$. This completes the proof. 
Theorem 1.2 and Propositions 3.1 and 3.3 yield the following corollary.

Corollary 3.4. Conjecture 1.1 holds as long as it holds when $M(\alpha)$ is an irreducible, atoroidal, small Seifert manifold.

Here is a result from [BGZ2]. Recall from $\S 6$ of that paper that $t_{j}^{+}$is the number of tight components of $\breve{\Phi}_{j}^{+}$.

A 3-manifold is very small if its fundamental group does not contain a nonabelian free group.

Proposition 3.5. Suppose that $F$ is a once-punctured essential genus 1 surface of boundary slope $\beta$ in a hyperbolic knot manifold $M$ which completes to an essential torus in $M(\beta)$ but is not a fibre in $M$. If $M(\alpha)$ is a small Seifert manifold, then

$$
\Delta(\alpha, \beta) \leq \begin{cases}6 & \text { if } M(\alpha) \text { is very small, } \\ 8 & \text { otherwise. }\end{cases}
$$

Moreover if $t_{1}^{+}>0$, then

$$
\Delta(\alpha, \beta) \leq \begin{cases}3 & \text { if } M(\alpha) \text { is very small, } \\ 4 & \text { otherwise. }\end{cases}
$$

Remark 3.6. When $t_{1}^{+}=0, M(\beta)_{\widehat{F}}$ is Seifert with base orbifold an annulus with one cone point [BGZ2, Lemma 7.9].

Proof of Proposition 3.5. The first inequality is the conclusion of BGZ2, Proposition 13.2]. To deduce the second we use the notation and results of [BGZ2.

Suppose next that $t_{1}^{+}>0$. Since $t_{1}^{+}$is even and the number of boundary components of $F$ is bounded below by $\frac{1}{2} t_{1}^{+}$, we have $t_{1}^{+}=2$. Proposition 13.1 of BGZ2 then shows that $\Delta(\alpha, \beta) \leq 4$. Suppose that $M(\alpha)$ is very small. The first paragraph of the proof of [BGZ2, Proposition 13.1] shows that $\Delta(\alpha, \beta) \leq 3$ if $\bar{\Gamma}_{S}$ has a vertex of valency 3 or less, while the second shows that the same inequality holds if it doesn't. This completes the proposition's proof.

\section{Involutions on SMALl Seifert manifolds}

We collect several results about involutions on small Seifert manifolds in this section.

Lemma 4.1. Let $W$ be a small Seifert manifold and $\tau$ an orientation-preserving involution on $W$ with non-empty fixed point set. Then there is a $\tau$-invariant Seifert structure on $W$ with base orbifold of the form $S^{2}(a, b, c)$, where $1 \leq a \leq b \leq c$.

Proof. If $W$ is a lens space, the result follows from [HR. Assume then that this isn't the case and fix a Seifert structure on $W$ with base orbifold $S^{2}(a, b, c)$, where $a \leq b \leq c$. The assumption that $\pi_{1}(W)$ is not cyclic implies that $a \geq 2$ and $a, b, c$ are determined by $W$.

Let $L \subset W / \tau$ be the branch set of $\tau$. The orbifold theorem implies that the orbifold $W / \tau$ is geometric, and since $L$ is a link, $W / \tau$ admits a Seifert structure with a 2-dimensional base orbifold $[\mathrm{Du}$. Thus $W$ admits a $\tau$-invariant Seifert structure. We claim that we can assume this structure has base orbifold $S^{2}(a, b, c)$. If $b \neq 2$, all Seifert structures on $W$ have this form, so assume $a=b=2 \leq c$. If the base orbifold of the $\tau$-invariant structure is not $S^{2}(a, b, c)$, it must be $P^{2}(d)$ for some integer $d \geq 1$. When $d>1$, there is a unique singular fibre $\phi$ in this 
structure, and it must be invariant under $\tau$. Then $\tau$ leaves the exterior $E$ of this fibre invariant, which is a twisted $I$-bundle over the Klein bottle. By assumption, $\tau$ leaves the Seifert structure on $E$ with base orbifold a Möbius band invariant. There is exactly one other Seifert structure on $E$, up to isotopy, and its base orbifold is $D^{2}(2,2)$. Moreover, there is at least one such structure which is $\tau \mid E$-invariant. This structure can be extended across a fibred neighbourhood of $\phi$ in a $\tau$-invariant fashion, yielding the desired $\tau$-invariant structure on $W$.

The argument is similar if $d=1$, for $\tau$ induces an involution of the base orbifold $P^{2}$ of $W$, and since any self-map of $P^{2}$ has a fixed point, there is a $\tau$-invariant fibre $\phi$ in $W$. Now proceed as in the case $d>1$.

For our next three results we let $W$ denote a small Seifert manifold and $\tau$ an orientation-preserving involution on $W$ with non-empty fixed point set such that the quotient $W / \tau$ is a lens space $L(\bar{p}, \bar{q}) \not S^{3}$. We use $L_{\tau}$ to denote the branch set of $\tau$ in $L(\bar{p}, \bar{q})$.

Fix a $\tau$-invariant Seifert structure on $W$ with base orbifold of the form $S^{2}(a, b, c)$ where $1 \leq a \leq b \leq c$ (Lemma 4.1) and let $\bar{\tau}$ be the involution of $S^{2}(a, b, c)$ (possibly the identity) induced by $\tau$.

Since the $\tau$-invariant Seifert structure on $W$ has an orientable base orbifold, its fibres can be coherently oriented.

Hodgson and Rubinstein have classified orientation-preserving involutions on lens spaces with non-empty fixed point sets. In particular, their work yields the following result.

Lemma $4.2([\mathrm{HR}, \S 4.7])$. Suppose that $W$ is the lens space $L(p, q)$ and $W / \tau=$ $L(\bar{p}, \bar{q}) \nRightarrow S^{3}$.

(1) If $p$ is odd, then $L_{\tau}$ is connected and is either

(a) the core of a solid torus of a genus one Heegaard splitting of $L(\bar{p}, \bar{q})$, or

(b) the boundary of a Möbius band spine of a Heegaard solid torus of $L(\bar{p}, \bar{q})$.

(2) If $p$ is even, then $L_{\tau}$ has two components and is either

(a) the union of the cores of the two solid tori of a genus one Heegaard splitting of $L(\bar{p}, \bar{q})$, or

(b) the boundary of an annular spine of a Heegaard solid torus of $L(\bar{p}, \bar{q})$.

Next we suppose that $W$ is not a lens space. In this case $2 \leq a \leq b \leq c$.

Lemma 4.3. Suppose that $W$ is not a lens space and that $\tau$ preserves the orientations of the Seifert fibres of $W$. Then there is an induced Seifert structure on $W / \tau$ such that $L_{\tau}$ is a union of at most three Seifert fibres where at least one of the fibres is regular. Further, $\bar{\tau}$ is either the identity or has two fixed points and

(1) if $\bar{\tau}$ is the identity then $a=2,\left|L_{\tau}\right|$ is the number of cone points of $S^{2}(a, b, c)$ of even order, and the components of $L_{\tau}$ which are regular fibres correspond to the cone points of order 2 ;

(2) if $\bar{\tau}$ is not the identity then $L_{\tau}$ has at most two components, and exactly one of its components is a regular fibre. 
Proof. The hypotheses imply that there is an induced Seifert structure on $L(\bar{p}, \bar{q})$ whose fibres are the images of the fibres of $W$. Since $W$ has three exceptional fibres, $\bar{\tau}$ fixes precisely one or three cone points. In the latter case, $\bar{\tau}$ is the identity.

Suppose first that $\bar{\tau}$ is the identity on $S^{2}(a, b, c)$. Since $\tau$ has 1-dimensional fixed point set, $\tau$ rotates the regular fibres of $W$ by $\pi$. Its fixed point set is the union of the fibres of even multiplicity, and therefore $L_{\tau}$ is a union of Seifert fibres. The reader will verify that if a fibre of $W$ has multiplicity $k$, then its image in $L(\bar{p}, \bar{q})$ has multiplicity $\bar{k}=\frac{k}{\operatorname{gcd}(k, 2)}$. Hence as $L(\bar{p}, \bar{q})$ has at most two exceptional fibres, $a=2$.

Suppose next that $\bar{\tau}$ fixes precisely one cone point of $S^{2}(a, b, c)$. In this case its fixed point set consists of this cone point and a regular point. Thus the fixed point set of $\tau$ is contained in a union of two fibres, so $L_{\tau}$ has at most two components. The reader will verify that each exceptional fibre of $W$ is sent to an exceptional fibre of $L(\bar{p}, \bar{q})$, two of them to the same fibre. Thus the $\tau$-invariant regular fibre of $W$ is sent to a regular fibre of $L(\bar{p}, \bar{q})$. It follows that this fibre lies in the fixed point set of $\tau$, and therefore $L_{\tau}$ contains a regular fibre of $L(\bar{p}, \bar{q})$.

Lemma 4.4. Suppose that $W$ is not a lens space and that $\tau$ reverses the orientations of the Seifert fibres of $W$. If $W / \tau=L(\bar{p}, \bar{q}) \neq S^{3}$, then

(1) $W$ has base orbifold $S^{2}(\bar{p}, \bar{p}, m)$, where $m \geq 2$ and the Seifert invariants of the exceptional fibres of order $\bar{p}$ are the same. Hence if $W$ is not a prism manifold, $\bar{p} \neq 2$.

(2) There is an integer $n$ coprime with $m$ such that $L_{\tau}$ is isotopic to the closure $K(m / n)$ of an $m / n$ rational tangle in a Heegaard solid torus of $W / \tau$ as depicted in Figure 1. In particular,

$$
\left|L_{\tau}\right|= \begin{cases}1 & \text { if } n \text { is odd } \\ 2 & \text { if } n \text { is even }\end{cases}
$$

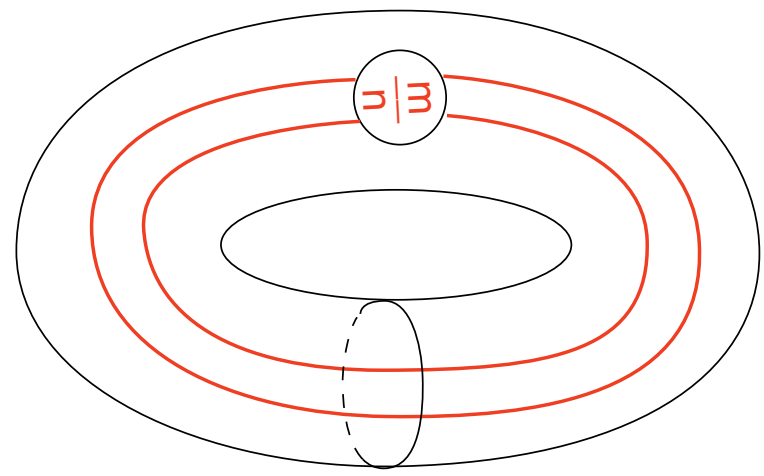

Figure 1

Proof. The fixed point set of $\bar{\tau}$ is non-empty, so as it reverses orientation, it is a reflection in an equator of $S^{2}(a, b, c)$. This equator cannot contain all three cone points, as otherwise $\tau$ would be the Montesinos involution on $W$ and therefore $L(\bar{p}, \bar{q})$ would be $S^{3}$. Thus it contains exactly one cone point and $\bar{\tau}$ permutes the other two. It follows that up to relabeling, $(a, b, c)=(r, r, m)$ for some integers $r, m \geq 2$. Further, $S^{2}(r, r, m) / \bar{\tau}=D^{2}(r ; m)$, where $D^{2}(r ; m)$ is the 2-orbifold with 
underlying space a 2-disk and singular set consisting of a cone point of order $r$, a corner-reflector point $x$ of order $m$, and a reflection line $\partial D^{2} \backslash\{x\}$. Therefore $L(\bar{p}, \bar{q})=W / \tau \cong L(r, t)$ for some integer $t$. Thus $r=\bar{p}$, which proves part (1).

A Montesinos-type analysis of the quotient of the $\tau$-invariant solid torus given by the inverse image in $W$ of a small annular neighbourhood of $\operatorname{Fix}(\bar{\tau})$ in $S^{2}(\bar{p}, \bar{p}, m)$ shows that the branch set of this quotient is of the form described in part (2). It is well known that this branch set has one component if $n$ is odd and two otherwise, so part (2) holds.

\section{Beginning of the proof of Theorem 1.3}

5.1. Assumptions. We assume throughout the rest of the paper that $M$ is a hyperbolic knot manifold containing an essential once-punctured torus $F$ of boundary slope $\beta$ which caps off to an essential torus in $M(\beta)$ (cf. Corollary 3.2) and that $M(\alpha)$ is an atoroidal, irreducible, small Seifert manifold (cf. Corollary [3.4). We assume as well that $\Delta(\alpha, \beta)>3$, and (therefore) $M_{F}$ is a genus 2 handlebody by Proposition 3.3 .

We will show that under these assumptions, $\Delta(\alpha, \beta) \leq 5, F$ is not a fibre, $\pi_{1}(M(\alpha))$ is finite non-cyclic, and

(a) if $\Delta(\alpha, \beta)=4,(M ; \alpha, \beta) \cong\left(W h\left(\frac{-2 n \pm 1}{n}\right) ;-4,0\right)$ for some integer $n$ with $|n|>1$ and $M(\alpha)$ has base orbifold $S^{2}(2,2,|\mp 2 n-1|)$;

(b) if $\Delta(\alpha, \beta)=5$, then $(M ; \alpha, \beta) \cong(W h(-3 / 2) ;-5,0)$ and $M(\alpha)$ has base orbifold $S^{2}(2,3,3)$.

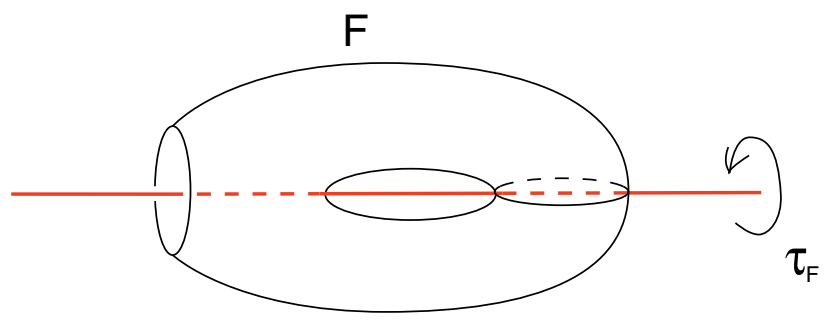

FIGURE 2

5.2. An involution on $M$. There is an involution $\tau_{F}$ on $F$ with exactly three fixed points whose action on $\partial F$ is rotation by $\pi$. See Figure 2 Thus $F / \tau_{F}$ is the 2-orbifold $D^{2}(2,2,2)$. Let $N \cong F \times I$ be a small neighbourhood of $F$ in $M$ and extend $\tau_{F}$ to an involution $\tau_{N}$ in the obvious way. Then $\tau_{N} \mid F \times \partial I$ extends to a hyperelliptic involution of $\partial M_{F}$. Since $M_{F}$ is a genus 2 handlebody, the latter extends to an involution $\tau_{M_{F}}$ of $M_{F}$. Piecing together $\tau_{N}$ and $\tau_{M_{F}}$ we obtain an orientation-preserving involution $\tau: M \rightarrow M$ with non-empty 1-dimensional fixed point set $\widetilde{L} \subset \operatorname{int}(M)$. Further, $V:=M / \tau$ is a solid torus containing the branch set $L$ of $\tau$. By construction, this is a hyperbolic link which intersects some meridional disk of $V$ transversely and in three points. When $F$ is a fibre in $M, L$ is braided in $V$.

Note that $L$ cannot intersect any meridional disk in one point, as $M$ is $\partial$ irreducible.

The slopes on $\partial M$ can be identified with \pm -classes of primitive elements of $H_{1}(\partial M)$. In particular we assume $\alpha, \beta \in H_{1}(\partial M)$. Let $\mu$ be any dual slope to $\beta$. 
This means that $1=\Delta(\mu, \beta)=|\mu \cdot \beta|$. Hence $\{\mu, \beta\}$ form a basis for $H_{1}(\partial M)$. Write

$$
\alpha=p \mu+q \beta,
$$

where $p, q$ are coprime. After possibly changing the signs of $\mu$ and $\beta$ we may assume that

$$
p=\Delta(\alpha, \beta) .
$$

Without loss of generality we may suppose that $p \geq 1$. The map $M \rightarrow V$ is a double cover when restricted to $\partial M$. It sends $\beta$ to a slope $\bar{\beta}$, a meridian of $V$, and sends $\mu$ to $\bar{\mu}$, a longitude of $V$.

For each slope $\gamma$ on $\partial M, \tau$ extends to an involution $\tau_{\gamma}: M(\gamma) \rightarrow M(\gamma)$. Moreover, if $\widetilde{U}_{\gamma}$ denotes the filling torus in $M(\gamma)$ and $\widetilde{K}_{\gamma}$ its core, then

$$
\operatorname{Fix}\left(\tau_{\gamma}\right)= \begin{cases}\widetilde{L} & \text { if } \Delta(\gamma, \beta) \text { is odd, } \\ \widetilde{L} \cup \widetilde{K}_{\gamma} & \text { if } \Delta(\gamma, \beta) \text { is even. }\end{cases}
$$

It is clear that $\widetilde{U}_{\gamma} / \tau_{\gamma}$ is a solid torus $U_{\gamma}$. Denote its core $\widetilde{K}_{\gamma} / \tau_{\gamma}$ by $K_{\gamma}$. Thus $M(\gamma) / \tau_{\gamma}=V \cup_{\bar{\gamma}} U_{\gamma}$ is a lens space. Indeed, if $\gamma=r \mu+s \beta$, then under the double cover $\partial M \rightarrow \partial V$ we have $\gamma \mapsto r \bar{\mu}+2 s \bar{\beta}$. Let $\bar{\gamma}=\frac{1}{\operatorname{gcd}(2, r)}(r \bar{\mu}+2 s \bar{\beta})$ denote the associated slope and $L_{\gamma}$ the branch set in $M(\gamma) / \tau_{\gamma}$. Then

$$
\left(M(\gamma) / \tau_{\gamma}, L_{\gamma}\right)=\left(V(\bar{\gamma}), L_{\gamma}\right) \cong \begin{cases}(L(r, 2 s), L) & \text { if } r \text { is odd } \\ \left(L\left(\frac{r}{2}, s\right), L \cup K_{\gamma}\right) & \text { if } r \text { is even. }\end{cases}
$$

We are interested in the case $\gamma=\alpha$. Set

$$
\bar{p}=p / \operatorname{gcd}(p, 2) \quad \text { and } \quad \bar{q}=2 q / \operatorname{gcd}(p, 2)
$$

so that $\bar{\alpha}=\bar{p} \bar{\mu}+\bar{q} \bar{\beta}$ and

$$
M(\alpha) / \tau_{\alpha} \cong L(\bar{p}, \bar{q})
$$

From 5.2 .3 we see that

$$
\left|L_{\alpha}\right|= \begin{cases}|L| & \text { if } p \text { is odd } \\ |L|+1 & \text { if } p \text { is even. }\end{cases}
$$

Fix a $\tau_{\alpha}$-invariant Seifert structure on $M(\alpha)$ with base orbifold $S^{2}(a, b, c)$ where $1 \leq a \leq b \leq c$ (Lemma 4.1)

Let $\bar{\tau}_{\alpha}$ be the involution of $S^{2}(a, b, c)$ (possibly the identity) induced by $\tau_{\alpha}$.

Lemma 5.1. Suppose that Assumptions 5.1 hold. Suppose as well that $M(\alpha)$ is not a lens space and that $\tau_{\alpha}$ preserves the orientations of the Seifert fibres of $M(\alpha)$. Then there is a Seifert structure on $L(\bar{p}, \bar{q})$ in which $L_{\alpha}$ is a union of at most three fibres, at least one of which is regular. Further, $L_{\alpha}=L$ so that $p=\Delta(\alpha, \beta)$ is odd.

Proof. Lemma 4.3 shows that $L$ is a union of fibres in the induced Seifert structure on $L(\bar{p}, \bar{q})$ and that at least one of these fibres is regular. This implies that $K_{\alpha} \not \subset L_{\alpha}$, as otherwise $L=L_{\alpha} \backslash K_{\alpha}$ would not be a hyperbolic link in $V$. Thus $L=L_{\alpha}$, so $p$ is odd by (5.2.5).

Lemma 5.2. Suppose that Assumptions 5.1 hold. Suppose as well that $M(\alpha)$ is not a lens space and that $\tau_{\alpha}$ reverses the orientations of the Seifert fibres of $M(\alpha)$. 
Then

(1) $M(\alpha)$ has base orbifold $S^{2}(\bar{p}, \bar{p}, m)$, where $m \geq 2$ and the Seifert invariants of the exceptional fibres of order $\bar{p}$ are the same. Hence if $M(\alpha)$ is not a prism manifold, $\Delta(\alpha, \beta) \neq 4$.

(2) There is an integer $n$ coprime with $m$ such that $L_{\alpha}$ is isotopic to the closure $K(m / n)$ of an $m / n$ rational tangle in a Heegaard solid torus of $M(\alpha) / \tau_{\alpha}$ as depicted in Figure 1. In particular,

$$
\left|L_{\alpha}\right|= \begin{cases}1 & \text { if } n \text { is odd } \\ 2 & \text { if } n \text { is even }\end{cases}
$$

(3) $|L|=1, m$ is odd, and $n \equiv p(\bmod 2)$.

Proof. Parts (1) and (2) follow from Lemma 4.4.

In order to prove part (3), suppose that $|L|=2$. Then part (2) shows that $L=L_{\alpha}$. In particular, $p$ is odd (5.2.5). Consideration of the form of $L_{\alpha}$ (cf. Figure 1) shows that its two components are isotopic to one another. But since $L$ is transverse to a meridian disk of $V$ and intersects it in three points, the generator $\gamma$ of $H_{1}(V(\bar{\alpha})) \cong \mathbb{Z} / \bar{p}$ carried by the core of $V$ satisfies $\gamma= \pm 2 \gamma$. Hence $\bar{p}=3$. But $p$ is odd so $\Delta(\alpha, \beta)=p=\bar{p}=3$, contrary to our hypotheses. Thus $|L|=1$.

Next suppose that $m$ is even. Then $L_{\alpha}=K(m / n)$ is connected, so $L=L_{\alpha}$ and $p$ is odd, and $L$ is homotopically trivial in $L(\bar{p}, \bar{q})$. But $L$ intersects a meridian disk of the Heegaard torus $V \subset L(\bar{p}, \bar{q})$ transversely and in three points, so the only way it can be null homotopic is for $3=\bar{p}$. Since $p$ is odd, $p=3$, which contradicts our hypotheses. Thus $m$ is odd.

By $(2),\left|L_{\alpha}\right| \equiv n(\bmod 2)$. Since $|L|=1$ by (3), Identity (5.2.5) shows that $\left|L_{\alpha}\right| \equiv p(\bmod 2)$.

5.3. Constraints on the branch set $L$. Here we deduce strong constraints on the form of the branch set $L$ in $V$.

Lemma 5.3. Suppose that Assumptions 5.1 hold and that $\tau_{\alpha}$ reverses the orientation of the Seifert fibres of $M(\alpha)$. Let $k \geq 1$ be an integer dividing $\bar{p}$ and consider the $k$-fold cyclic cover $S^{2}\left(\frac{\bar{p}}{k}, \frac{\bar{p}}{k}, m, m, \ldots, m\right) \rightarrow S^{2}(\bar{p}, \bar{p}, m)$ obtained by the $k$-fold unwrapping of $S^{2}(\bar{p}, \bar{p}, m)$ about the two cone points labeled $\bar{p}$. Let $\widetilde{M(\alpha)_{k}} \rightarrow M(\alpha)$ be the associated $k$-fold cyclic cover where $\widetilde{M(\alpha)_{k}}$ is Seifert with base orbifold $S^{2}\left(\frac{\bar{p}}{k}, \frac{\bar{p}}{k}, m, m, \ldots, m\right)$ and the inclusion of a regular fibre of $M(\alpha)$ lifts to $\left.\widetilde{M(\alpha)}\right)_{k}$. Define $\widetilde{M}_{k} \rightarrow M$ to be the cover obtained by restricting $\widetilde{M(\alpha)_{k}} \rightarrow M(\alpha)$ to $M$. Then

(1) $\partial \widetilde{M}_{k}$ is connected and $F$ lifts to $\widetilde{M}_{k}$. In particular, $\beta$ lifts to a slope $\widetilde{\beta}$ on $\partial \widetilde{M}_{k}$.

(2) $\alpha$ lifts to a slope $\widetilde{\alpha}$ on $\partial \widetilde{M}_{k}$ such that $\widetilde{M(\alpha)_{k}}=\widetilde{M}_{k}(\widetilde{\alpha})$. Further, $\Delta(\widetilde{\alpha}, \widetilde{\beta})=\frac{p}{k}$.

(3) $\widetilde{\alpha}$ is the singular slope of a closed essential surface in $\widetilde{M}_{k}$ if $S^{2}\left(\frac{\bar{p}}{k}, \frac{\bar{p}}{k}, m, m, \ldots\right.$, $m)$ is hyperbolic with at least four cone points. If this is the case, $p / k \leq 3$.

Proof. The cover $S^{2}\left(\frac{\bar{p}}{k}, \frac{\bar{p}}{k}, m, m, \ldots, m\right) \rightarrow S^{2}(\bar{p}, \bar{p}, m)$ is determined by the homomorphism $\varphi: H_{1}\left(S^{2}(\bar{p}, \bar{p}, m)\right)=\langle x, y: \bar{p} x=\bar{p} y=m(x+y)=0\rangle \rightarrow \mathbb{Z} / k$, where $\varphi(x) \equiv-\varphi(y) \equiv 1(\bmod k)$.

First note that the homomorphism $H_{1}(M(\alpha)) \rightarrow H_{1}(V(\bar{\alpha})) \cong \mathbb{Z} / \bar{p}$ kills any class carried by a regular Seifert fibre of $M(\alpha)$ (i.e. there are regular fibres with 
image an interval). Thus it factors through a homomorphism $\psi: H_{1}\left(S^{2}(\bar{p}, \bar{p}, m)\right) \rightarrow$ $H_{1}(V(\bar{\alpha}))$. Since $\tau_{\alpha}$ preserves the fibre of multiplicity $m$ in $M(\alpha)$ but reverses its orientation, $\left(\bar{\tau}_{\alpha}\right)_{*}(x+y)=-(x+y)$. Thus $2(x+y)$ is sent to zero in $H_{1}(V(\bar{\alpha}))$, while $x$ is sent to a generator. Since $m$ is odd and $m(x+y)=0, x+y \mapsto 0 \in H_{1}(V(\bar{\alpha}))$.

It follows that $\varphi$ factors as $H_{1}\left(S^{2}(\bar{p}, \bar{p}, m)\right) \stackrel{\psi}{\longrightarrow} H_{1}(V(\bar{\alpha})) \stackrel{\cong}{\longrightarrow} / \bar{p} \rightarrow \mathbb{Z} / k$. Since $H_{1}(F)$ lies in the kernel of $H_{1}(M) \rightarrow H_{1}(V)$ while $\mu$ is sent to a generator of $H_{1}(V)$, we conclude that $\partial \widetilde{M}_{k}$ is connected and $F$ lifts to $\widetilde{M}_{k}$. This proves (1).

For $(2)$, note that by construction, there is a basis $\{\widetilde{\mu}, \widetilde{\beta}\}$ of $H_{1}\left(\partial \widetilde{M}_{k}\right)$ where $\widetilde{\mu}$ is sent to $k \mu$ and $\widetilde{\beta}$ is sent to $\beta$ in $H_{1}(\partial M)$. Then $\alpha=p \mu+q \beta$ lifts to $\left(\frac{p}{k}\right) \widetilde{\mu}+q \widetilde{\beta}$. Clearly $\Delta(\widetilde{\alpha}, \widetilde{\beta})=\frac{p}{k}$.

Part (3) is a consequence of [BGZ1, Theorems 1.5 and 1.7].

Lemma 5.4. Suppose that Assumptions 5.1 hold. Then $M$ is not a once-punctured torus bundle. In particular, Theorem 1.3 holds when $F$ is a fibre.

Proof. We assume that $M$ is a once-punctured torus bundle in order to obtain a contradiction.

There is a 3-braid $\sigma$ whose closure in $V$ is $L$. Altering $\sigma$ by conjugation in $B_{3}=\left\langle\sigma_{1}, \sigma_{2}: \sigma_{1} \sigma_{2} \sigma_{1}=\sigma_{2} \sigma_{1} \sigma_{2}\right\rangle$ leaves its closure invariant. (Here $\sigma_{1}, \sigma_{2}$ are the standard generators of $B_{3}$.) There is an isomorphism $B_{3} \cong\left\langle a, b: a^{3}=b^{2}\right\rangle$ where $a=\sigma_{1} \sigma_{2}$ and $b=\sigma_{1} \sigma_{2} \sigma_{1}$. The center of $B_{3}$ is generated by $a^{3}$ with $B_{3} /\left\langle a^{3}\right\rangle \cong$ $\mathbb{Z} / 2 * \mathbb{Z} / 3$. We will use $\bar{\sigma}$ to denote the image of a braid $\sigma$ in $B_{3} /\left\langle a^{3}\right\rangle$. Thus $\bar{a}$ has order 3 and $\bar{b}$ has order 2 . In particular,

$$
\begin{gathered}
\bar{\sigma}_{1}=\bar{a}^{-1} \bar{b}, \\
\bar{\sigma}_{2}=\bar{b} \bar{a}^{2} .
\end{gathered}
$$

The inverse image $\widehat{L}$ of $L \subset V \subset L(\bar{p}, \bar{q})$ under the universal cover $S^{3} \rightarrow L(\bar{p}, \bar{q})$ is the closure of the braid $\sigma^{\bar{p}} a^{-3 \bar{q}}$.

Claim 5.5. $\widehat{L}$ is not the trivial knot.

Proof of Claim 5.5. If $\widehat{L}$ is trivial then $\sigma^{\bar{p}} a^{-3 \bar{q}}$ is conjugate to $\sigma_{1} \sigma_{2}, \sigma_{1}^{-1} \sigma_{2}^{-1}$, or $\sigma_{1} \sigma_{2}^{-1}$ ([BiMe, Classification Theorem, page 27]). The first two cases can be ruled out since they would imply that the exterior of $\widehat{L}$ in the inverse image of $V$ in $S^{3}$ is not hyperbolic. On the other hand, in the third case we have $\bar{\sigma}^{\bar{p}}=\bar{\sigma}_{1} \bar{\sigma}_{2}^{-1}=$ $\bar{a}^{2} \bar{b} \bar{a} \bar{b} \in B_{3} /\left\langle a^{3}\right\rangle \cong \mathbb{Z} / 2 * \mathbb{Z} / 3$. But this is impossible since $\bar{a}^{2} \bar{b} \bar{a} \bar{b}$ is not a proper power.

Claim 5.6. $\tau_{\alpha}$ preserves the orientation of the Seifert fibres of $M(\alpha)$. In particular, $\widehat{L}$ is a union of fibres in some Seifert structure on $S^{3}$ and $p$ is odd.

Proof of Claim 5.6. Suppose otherwise and consider the $\bar{p}$-fold cyclic cover $\widetilde{M}_{\bar{p}} \rightarrow$ $M$ constructed in Lemma 5.3 The base orbifold $S^{2}(m, m, \ldots, m)$ of $\widetilde{M(\alpha)}$ has $\bar{p}$ cone points, each of order $m \geq 3$ by Lemma $5.2(3)$. If $\bar{p} \geq 4$, Lemma 5.3. (3) implies that $\widetilde{M}_{\bar{p}}$ contains a closed essential surface, contrary to [JR or $[\mathrm{FH}$. Hence $\bar{p}$ is 2 or 3 , and therefore as $p>3, p$ is 4 or 6 . Identity (5.2.5) then combines with parts (2) and (3) of Lemma 5.2 to show that $\left|L_{\alpha}\right|=2$ and $m$ is odd. It follows that each component of $L_{\alpha}$ is isotopic to the core of a Heegaard solid torus in $L(\bar{p}, \bar{q})$ (cf. Figure 11). In particular this is true of $L=L_{\alpha} \backslash K_{\alpha}$. It follows that $\widehat{L}$ is a trivial 
knot, contrary to the conclusion of Claim 5.5. Thus $\tau_{\alpha}$ preserves the orientation of the Seifert fibres of $M(\alpha)$. The remaining conclusions are a consequence of Lemma 5.1 .

Claim [5.6 implies that $\bar{p}=p$ and $\bar{q}=2 q$.

Since $L$ is a hyperbolic link in $V, \widehat{L}$ is a hyperbolic link in the inverse image of $V$ in $S^{3}$. Thus the Schreier normal form for $\sigma^{p} a^{-6 q}$ is generic (cf. [FKP, Theorem 5.2]). On the other hand, by Claim [5.6, $\widehat{L}$ is not a hyperbolic link in $S^{3}$, so FKP, Theorem 5.5] implies that $\sigma^{p} a^{-6 q}$ is conjugate in $B_{3}$ to a braid of the form $\sigma_{1}^{c} \sigma_{2}^{d}$ where $c, d \in \mathbb{Z} \backslash\{0\}$. We must have $\min \{|c|,|d|\}=1$, as otherwise $\widehat{L}$ would be a connected sum of non-trivial torus links, contrary to the conclusion of Claim 5.6. Thus $\sigma^{p} a^{-6 q}$ is conjugate to $\sigma_{1}^{c} \sigma_{2}^{\epsilon}$ for some $\epsilon \in\{ \pm 1\}$ and non-zero $c$. The following claim completes the proof of Lemma 5.4.

Claim 5.7. If $p>3, \sigma^{p} a^{-6 q}$ is not conjugate to $\sigma_{1}^{c} \sigma_{2}^{\epsilon}$ for any $\epsilon \in\{ \pm 1\}$.

Proof of Claim 5.7. Suppose that $\sigma^{p} a^{-6 q}$ is conjugate to $\sigma_{1}^{c} \sigma_{2}^{\epsilon}$ for some $\epsilon \in\{ \pm 1\}$. Projecting into $B_{3} /\left\langle a^{3}\right\rangle$ shows that $\bar{\sigma}_{1}^{c} \bar{\sigma}_{2}^{\epsilon}$ is a $p^{t h}$-power in that group. The latter condition is invariant under conjugation and taking inverse, so without loss of generality we can suppose that $\epsilon=1$. Now

$$
\bar{\sigma}_{1}^{c} \bar{\sigma}_{2}=\left(\bar{a}^{-1} \bar{b}\right)^{c}\left(\bar{b} \bar{a}^{-1}\right)= \begin{cases}(\bar{b} \bar{a})^{|c|}\left(\bar{b} \bar{a}^{-1}\right) & \text { if } c \leq 0 \\ \bar{a} & \text { if } c=1 \\ \bar{a}^{-1} \bar{b} \bar{a} & \text { if } c=2 \\ \left(\bar{a}^{-1} \bar{b}\right) \bar{a}^{-1}\left(\bar{a}^{-1} \bar{b}\right)^{-1} & \text { if } c=3 \\ (\bar{a}-1 \bar{b} \bar{a})(\bar{a} \bar{b})\left(\bar{a}^{-1} \bar{b}\right)^{c-4}\left(\bar{a}^{-1} \bar{b} \bar{a}\right)^{-1} & \text { if } c>3\end{cases}
$$

Consideration of the normal form for elements of $\mathbb{Z} / 2 * \mathbb{Z} / 3$ shows that the only values of $c$ which give proper powers in $B_{3} /\left\langle a^{3}\right\rangle$ are $c=1,2$, or 3 .

Say $c=1$ or 3 . Then up to conjugation, $\bar{\sigma}^{p}=\bar{a}^{ \pm 1}$, and therefore $\bar{\sigma}=\bar{a}^{ \pm 1}$. Hence $\sigma=a^{3 k \pm 1}$ for some integer $k$. But then it is easy to see that $L$ is boundary-parallel in $V$, contrary to the fact that $V \backslash L$ is hyperbolic.

Next suppose that $c=2$. Then $\bar{\sigma}^{p}=\bar{b}$ up to conjugation, and therefore the same is true of $\bar{\sigma}$. As $a^{3}=b^{2}, \sigma=b^{2 n+1}$ for some integer $n$. Then $L \subset \operatorname{int}(V)$ has two components. One is a core curve $K_{0}$ of $V$, while the other is isotopic in $V \backslash K_{0}$ into $\partial V$. It follows that there is an essential annulus properly embedded in the exterior of $L$ in $\operatorname{int}(V)$. But this contradicts the fact that $L$ is a hyperbolic link in $V$.

(of Lemma 5.4)

Recall that $t_{1}^{+}$is the number of tight components of $\breve{\Phi}_{1}^{+}$(cf. [BGZ2, $\left.\S 6\right]$ ).

Lemma 5.8. Suppose that Assumptions 5.1 hold. Then $t_{1}^{+}=0$. In particular, $M(\beta)_{\widehat{F}}$ is Seifert with base orbifold of the form $A(a)$, where $A$ is an annulus and $a \geq 2$.

Proof. Lemma 5.4 implies that $F$ is not a fibre, and so Proposition 3.5 and Remark 3.6. show that the lemma holds as long as either $M(\alpha)$ is very small or $\Delta(\alpha, \beta)>4$. Assume then that $M(\alpha)$ is not very small and that $\Delta(\alpha, \beta)=4$. The latter equality combines with Lemma 5.1 to show that $\tau_{\alpha}$ reverses the orientations of the fibres of $M(\alpha)$. But then Lemma $5.2(1)$ implies that $M(\alpha)$ is a prism manifold, contradicting our assumption that $M(\alpha)$ is not very small. Thus the lemma holds. 
Lemma 5.9. Suppose that Assumptions 5.1 hold. Then there are coprime integers $a \geq 2$ and $b$ as well as a 3-braid $\sigma$ such that $L$ is isotopic to the link depicted in Figure 3 ,

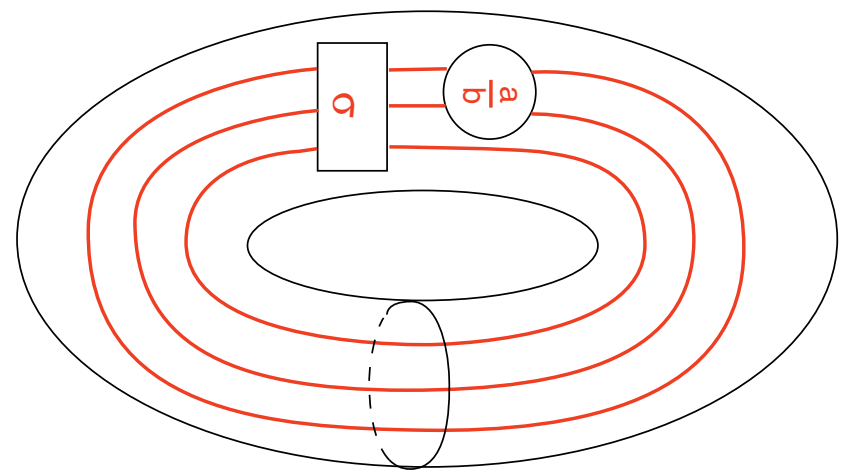

FiguRE 3

Proof. By Lemma 5.8, $M(\beta)_{\widehat{F}}$ is Seifert with base orbifold of the form $A(a)$, where $A$ is an annulus and $a \geq 2$. Consider the involution $\widehat{\tau}: M(\beta)_{\widehat{F}} \rightarrow M(\beta)_{\widehat{F}}$ induced by $\tau_{\beta}$. Note that $M(\beta)_{\widehat{F}} / \widehat{\tau}=V(\bar{\beta})_{\widehat{F} / \widehat{\tau}} \cong\left(S^{2} \times S^{1}\right)_{S^{2} \times\{x\}} \cong S^{2} \times I$. Now $M(\beta)_{\widehat{F}}$ has a unique Seifert structure which we can suppose is $\widehat{\tau}$-invariant. Let $\overline{\widehat{\tau}}$ be the induced involution on $A(a)$. Note that $\overline{\widehat{\tau}}$ cannot preserve orientation, as otherwise $M(\beta)_{\widehat{F}} / \widehat{\tau} \cong S^{2} \times I$ would admit a Seifert structure. Thus it reverses orientation, and since it fixes the cone point and leaves each boundary component invariant, it must be reflection along a pair of disjoint properly embedded arcs, each of which runs from one boundary component to the other. The quotient $A(a) / \overline{\widehat{\tau}}$ is a disk whose boundary contains two disjoint, compact arcs, each a reflector arc, one of which contains the $\mathbb{Z} / a$ cone point. It follows that the branch set in $M(\beta)_{\widehat{F}} / \widehat{\tau} \cong S^{2} \times I$ consists of a 2-braid and an $\frac{a}{b}$-rational tangle running from one end to the other which are separated by a properly embedded vertical annulus. See Figure 4 ,

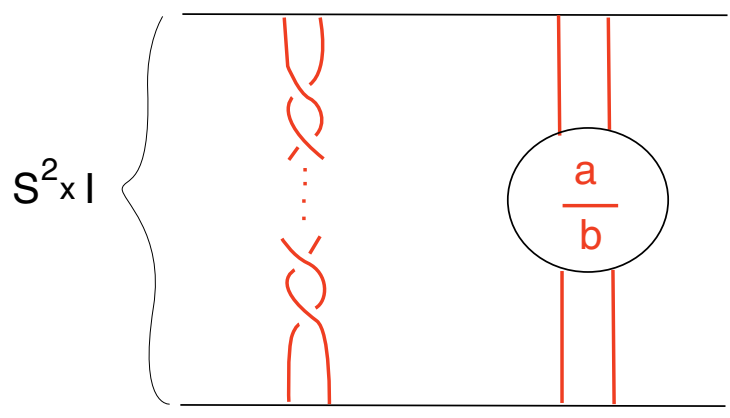

Figure 4

We claim that $K_{\beta} \cap M(\beta)_{\widehat{F}}$ is a component of the 2-braid. To see this, first note that by Lemma 5.8, $\breve{\Phi}_{1}^{+}$has no tight components. Next we refer the reader to the final paragraph of the proof of [BGZ2, Lemma 7.9]. It is shown there that 
$M_{F}=X^{+}$is obtained by attaching a solid torus $V$ to the product of an interval $I$ and a once-punctured annulus $A_{*}$, where $V \cap\left(A_{*} \times I\right)$ is a pair of annuli which have winding number $a$ in $V$ and components of $\partial A_{*} \times I$ in $A_{*} \times I$. This decomposition is invariant under the restriction of $\hat{\tau}$ to $M_{F}$, and it is easy to see that the quotient of $V$ contains the $\frac{a}{b}$-rational tangle. Since $(\partial M)_{\partial F} \subset A_{*} \times I$ is disjoint from $V$, it follows that $K_{\beta} \cap M(\beta)_{\widehat{F}}$ is a component of the 2-braid. Thus $L \cap M_{F} / \tau$ is as depicted in Figure 5, where $\delta$ is a 3-braid. It follows that there is a 3-braid $\sigma$ such that $L$ is as depicted in Figure 3 .

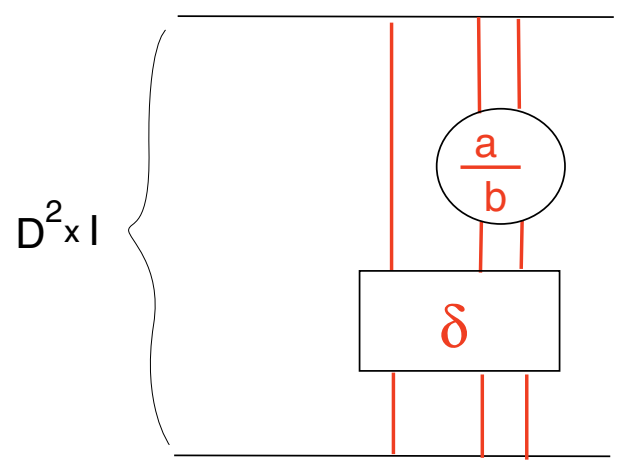

FiguRE 5

5.4. The lens space case. The methods of this paper can be used to give a new proof of Ken Baker's theorem: if $M$ contains a once-punctured essential genus 1 surface of boundary slope $\beta$ and $M(\alpha)$ is a lens space, then $\Delta(\alpha, \beta) \leq 3[\mathrm{Ba}$. We begin the proof here and complete it in $\$ 8$.

Lemma 5.10. Suppose that Assumptions 5.1 hold. If $\pi_{1}(M(\alpha))$ is cyclic, then $p=5, F$ is not a fibre, and $L_{\alpha}$ is either the core of a solid torus of a genus one Heegaard splitting of $L(5,2 q)$ or the boundary of a Möbius band spine of a Heegaard solid torus of $L(5,2 q)$.

Proof. We know that $F$ is not a fibre (Lemma 5.4), so $p=\Delta(\alpha, \beta) \leq 6$ by Proposition 3.5. As $\Delta(\alpha, \beta)=p \geq 4, M(\alpha) / \tau_{\alpha} \cong L(\bar{p}, \bar{q})$ is not $S^{3}$. Hence by Lemma 4.2. $L_{\alpha}$ is a union of Seifert fibres of some Seifert fibring of $L(\bar{p}, \bar{q})$. Since $L$ is hyperbolic in $V, K_{\alpha}$ cannot be contained in $L_{\alpha}$. Thus $p$ is odd by (5.2.3), so $p=\bar{p}=5, \bar{q}=2 q$, and $L=L_{\alpha}$. Lemma 4.2(1) then shows that $L_{\alpha}$ is either the core of a solid torus of a genus one Heegaard splitting of $L(5,2 q)$ or the boundary of a Möbius band spine of a Heegaard solid torus of $L(5,2 q)$.

Remark 5.11. We can complete the proof of Baker's result mentioned above at this point by invoking a theorem of Sangyop Lee [L3] which states that the distance between a toroidal filling slope and a lens space filling slope is at most 4 . Nevertheless, we give an independent proof that $\Delta(\alpha, \beta) \neq 5$ (and so $\Delta(\alpha, \beta) \leq 3)$ in 8 below. 
5.5. Reduction of the proof of Theorem 1.3. In this section we reduce the proof of Theorem 1.3 to several problems concerning links. These will be solved in the subsequent sections of the paper. We begin with a slight sharpening of our upper bound for $\Delta(\alpha, \beta)$.

Lemma 5.12. If Assumptions 5.1 hold, then $\Delta(\alpha, \beta)<8$.

Proof. By Lemma 5.4, $F$ is not a fibre in $M$. Hence $\Delta(\alpha, \beta) \leq 8$ by Proposition 3.5 (or [LM]). Suppose that $\Delta(\alpha, \beta)=8$. Then $M(\alpha)$ is not very small by Proposition 3.5. Further, Proposition 3.3 implies that $M_{F}$ is a genus two handlebody, so we can construct an involution $\tau$ as above. Then Lemma 5.1 implies that $\tau_{\alpha}$ reverses the orientations of the Seifert fibres of $M(\alpha)$. Parts (1) and (3) of Lemma 5.2 imply that $M(\alpha)$ has a Seifert structure with base orbifold $S^{2}(4,4, m)$, where $m \geq 3$ is odd. Let $\widetilde{M}_{2} \rightarrow M$ be the 2-fold cover constructed in Lemma 5.3. By part (2) of that lemma, $\widetilde{M}_{2}(\widetilde{\alpha})$ is Seifert with base orbifold $S^{2}(4,4, m, m)$. But then Lemma $5.3(3)$ implies $4=\frac{8}{2} \leq 3$, which is false. Thus $\Delta(\alpha, \beta) \neq 8$.

Lemma 5.13. Suppose that Assumptions 5.1 hold and that $\Delta(\alpha, \beta)=4$. Then $M(\alpha)$ is a prism manifold.

Proof. Since $\Delta(\alpha, \beta)$ is even, $M(\alpha)$ is not a lens space (Lemma 5.10), and so Lemma 5.1 implies that $\tau_{\alpha}$ reverses the orientations of the fibres of $M(\alpha)$. Lemma 5.2 (1) now implies that $M(\alpha)$ is a prism manifold.

Given the last two lemmas, to complete the proof of Theorem 1.3 under Assumptions [5.1. we must consider the possibility that $\Delta(\alpha, \beta) \in\{5,6,7\}$ besides the case when $\Delta(\alpha, \beta)=4$ and $M(\alpha)$ is a prism manifold. We do this by comparing the constraints obtained above on the branch sets $L$ and $L_{\alpha}$ :

- $L$ lies in $V$ as depicted in Figure 3 (Lemma 5.9);

- when $M(\alpha)$ is not a lens space and $\tau_{\alpha}$ preserves the orientation of the Seifert fibres of $M(\alpha)$, then $\Delta(\alpha, \beta)$ is odd and $L_{\alpha}$ is the union of at most three fibres of some Seifert structure on $L(\bar{p}, \bar{q})$ (Lemma 5.1);

- when $M(\alpha)$ is not a lens space and $\tau_{\alpha}$ reverses the orientation of the Seifert fibres of $M(\alpha)$, then $L_{\alpha}$ lies in some Heegaard solid torus of $L(\bar{p}, \bar{q})$ as depicted in Figure 1 (Lemma 4.4);

- when $M(\alpha)$ is a lens space, then $\Delta(\alpha, \beta)=5$ and $L_{\alpha}$ is either the core of a Heegaard solid torus of $L(5,2 q)$ or the boundary of a Möbius band spine of a Heegaard solid torus of $L(5,2 q)$ (Lemma 5.10).

The proof of Theorem 1.3 therefore reduces to proving the following claims:

(1) If $\tau_{\alpha}$ preserves the orientation of the Seifert fibres and $M(\alpha)$ is not a lens space, then $\Delta(\alpha, \beta)=5$ and $(M ; \alpha, \beta)$ is homeomorphic to $(W h(-3 / 2)$; $-5,0)$.

(2) The links contained in the universal cover $S^{3}$ of $L(7, \bar{q})$ which are depicted in Figure 17 and Figure 18 are not equivalent when $\Delta(\alpha, \beta)=7,|L|=1$, $m$ is odd, and $n \equiv 1(\bmod 2)$.

(3) The link depicted in Figure 3 considered as lying in a Heegaard solid torus in $L(5,2 q)$ is not isotopic to either the core of a Heegaard solid torus or the boundary of a Möbius band spine of a Heegaard solid torus.

(4) The links contained in a Heegaard solid torus in $L(3, \bar{q})$ depicted in Figure 1 and Figure 3 are not equivalent. 
(5) The links contained in the universal cover $S^{3}$ of $L(5, \bar{q})$ which are depicted in Figure 26] and Figure 27 are not equivalent when $\Delta(\alpha, \beta)=5,|L|=1$, $m$ is odd, and $n \equiv 1(\bmod 2)$.

(6) $\Delta(\alpha, \beta)=4$ and $M(\alpha)$ is a prism manifold if and only if $(M ; \alpha, \beta) \cong$ $\left(W h\left(\frac{-2 n \pm 1}{n}\right) ;-4,0\right)$ for some integer $n$ with $|n|>1$.

These will be proved in $₫ 6, \$ 7,98, \$ 9,910$ and $\$ 12$, respectively.

6. The CASE Where $\tau_{\alpha}$ Preserves the orientation of the SEIfERT Fibres, $M(\alpha)$ IS NOT A LENS SPACE, AND $\Delta(\alpha, \beta) \in\{5,7\}$

In this section we suppose that Assumptions 5.1 hold and show that if $\tau_{\alpha}$ preserves the orientation of the Seifert fibres, $M(\alpha)$ is not a lens space, and $\Delta(\alpha, \beta) \in$ $\{5,7\}$, then $\Delta(\alpha, \beta)=5$ and $(M ; \alpha, \beta)$ is homeomorphic to $(W h(-3 / 2) ;-5,0)$.

By hypothesis, $M(\alpha)$ is small Seifert with exactly three singular fibres. It is not a prism manifold by L2 and so has a unique Seifert structure. Recall that $M(\alpha) / \tau_{\alpha}=V(\bar{\alpha})$ is the lens space $L(\bar{p}, \bar{q})=L(p, 2 q)$ and the branch set of $\tau_{\alpha}$ in $L(p, 2 q)$ is a link denoted by $L_{\alpha}$. As $p$ is odd, $L_{\alpha}=L$ (cf. (5.2.5)).

Suppose that $L_{\alpha}$ is a Seifert link with respect to the induced Seifert fibration on $L(p, 2 q)=M(\alpha) / \tau_{\alpha}$. We need to show that $p=5$ and $(M ; \alpha, \beta)$ is homeomorphic to $(W h(-3 / 2) ;-5,0)$.

By Lemma [5.1, at least one component of $L$ is a regular fibre of $L(p, 2 q)$. Let $K$ be such a component and denote by $X$ the exterior of $L$ in $L(p, 2 q)$. Then $X$ has the induced Seifert fibration with $|\partial X|=|L|$ boundary components, each a torus. Let $T_{K}$ be the component of $\partial X$ corresponding to the knot $K$.

Lemma 6.1. There is an essential separating vertical annulus $(A, \partial A) \subset\left(X, T_{K}\right)$ which cuts $X$ into two components $X_{1}$ and $X_{2}$ such that each $X_{i}$ is either a torus cross interval or a fibred solid torus whose core is a singular fibre of $X$ of order larger than 2.

Proof. The lemma follows from Lemma 4.3 and its proof. Let $\bar{\tau}_{\alpha}$ be the induced map on the orbifold $S^{2}(a, b, c)$ of $M(\alpha)$ where each of $a, b, c$ is $\geq 2$. Then $\bar{\tau}_{\alpha}$ is either the identity or an involution with two fixed points. Let $\sigma_{1}, \sigma_{2}, \sigma_{3}$ denote the singular fibres of $M(\alpha)$ and let their orders be $a, b, c$, respectively.

First assume that $\bar{\tau}_{\alpha}$ is the identity map. Then Lemma 4.3) (1) implies that at least one of $a, b, c$, say $a$, is 2 and the fixed point set of $\tau_{\alpha}$ in $M(\alpha)$ is the union of those $\sigma_{i}$ with even orders. In particular, $\sigma_{1}$ belongs to the fixed point set of $\tau_{\alpha}$ and its image in $L(p, 2 q)$ is a regular fibre. Note that if $\sigma_{2}$, respectively $\sigma_{3}$, does not belong to the fixed point set of $\tau_{\alpha}$, then $b$, respectively $c$, is odd and the image of $\sigma_{2}$, respectively $\sigma_{3}$, in $L(p, 2 q)$ is a fibre of $L(p, 2 q)$ of order $b$, respectively $c$. Hence the sum of $|\partial X|=|L|$ and the number of the singular fibres of $X$ equals 3. Since the surface underlying the base orbifold of $X$ is planar, the lemma follows in this case.

Next assume that $\bar{\tau}_{\alpha}$ is an involution. Then two of the singular fibres of $M(\alpha)$, say $\sigma_{1}$ and $\sigma_{2}$, have the same order $a=b$. Both are mapped to a common singular fibre in $L(p, 2 q)$ of order $a$. Since $M(\alpha)$ is not a prism manifold, $a=b>2$.

By Lemma 4.3 $(2)$, the fixed point set of $\tau_{\alpha}$ in $M(\alpha)$ consists of a regular fibre and possibly the remaining singular fibre $\sigma_{3}$. If $\sigma_{3}$ does not belong to $\operatorname{Fix}\left(\tau_{\alpha}\right)$, then its image in $L(p, 2 q)$ is a singular fibre of order $2 c \geq 4$ and therefore the sum of 
$|\partial X|=|L|$ and the number of the singular fibres of $X$ again equals 3 . As in the previous case, the lemma follows from this.

Recall that $K_{\alpha}$ is the core circle of the filling solid torus in $V(\bar{\alpha})=L(p, 2 q)$. The exterior $Y$ of $K_{\alpha}$ in $X$ is also the exterior of $L$ in $V$ and so is hyperbolic. Let $T_{V}=\partial V \subset \partial Y$.

The solid torus $V$ has a meridian disk $D$ which intersects $L$ in three points such that $P=D \cap Y$ is an essential thrice-punctured disk in $Y$. Let $d_{V}=\partial P \cap T_{V}$ and let $c_{1}, c_{2}, c_{3}$ be the three components of $\partial P$ contained in $\partial Y \backslash T_{V}$. Note that $d_{V}$ has the slope $\bar{\beta}$ in $T_{V}$, and each $c_{i}$ is a meridian curve of some component of $L$.

Among all annuli satisfying the conditions of Lemma 6.1 we choose one, denoted $A$, which intersects $T_{V}$ in the minimal number of components. Since $Y$ is hyperbolic, $A \cap T_{V}$ is non-empty. The surface $Q=A \cap Y$ is essential in $Y$. Since $A$ is separating in $X, \partial Q \cap T_{V}$ consists of an even number, say $n$, of simple essential loops in $T_{V}$ of slope $\bar{\alpha}$. Let $a_{1}, a_{2}$ be the two components of $\partial Q$ in $T_{K}$, and let $b_{1}, \ldots, b_{n}$ be the components of $\partial Q$ in $T_{V}$ numbered so that they occur successively around $d_{V}$. Each $a_{i}$ is a Seifert fibre of $X$, and each $b_{j}$ has slope $\bar{\alpha}$ on $T_{V}$. If $c_{j}$ is a meridian curve of $K$, then the distance between $c_{j}$ and $a_{i}$ is 1 since $K$ is a regular fibre of $L(p, 2 q)$

Now define the labeled intersection graphs $\Gamma_{P}$ and $\Gamma_{Q}$ as usual. We may consider $d_{V}, c_{1}, c_{2}, c_{3}, a_{1}, a_{2}, b_{1}, \ldots, b_{n}$ as the boundaries of the fat vertices of these graphs. Each $b_{i}, i=1, \ldots, n$, has valency $p=\Delta(\bar{\alpha}, \bar{\beta})=\Delta(\alpha, \beta)$, and the valency of $d_{V}$ is $n p$. Note that the valency of $a_{1}$ is equal to the valency of $a_{2}$ and is equal to the number of $c_{i}$ 's which are meridians of $K$. Further, the valency of $c_{i}$ is either 2 or 0 depending on whether or not $c_{i}$ is a meridian curve of $K$.

We call the edges in $\Gamma_{Q}$ connecting some $b_{i}$ to some $b_{j} B$-edges, and call the edges in $\Gamma_{P}$ connecting $d_{V}$ to itself $D$-edges. Similarly we define $A$-edges, $C$-edges, $A B$-edges, and $C D$-edges. Note that an arc in $P \cap Q$ is a $B$-edge in $\Gamma_{Q}$ if and only if it is a $D$-edge in $\Gamma_{P}$, is an $A$-edge in $\Gamma_{Q}$ if and only if it is a $C$-edge in $\Gamma_{P}$, and is an $A B$-edge in $\Gamma_{Q}$ if and only if it is a $C D$-edge in $\Gamma_{P}$.

Every $D$-edge is positive, so by the parity rule, every $B$-edge is negative. By construction, no $D$-edge in $\Gamma_{P}$ is boundary parallel in $P$. Thus there are at most three different $D$-edges in the reduced graph $\bar{\Gamma}_{P}$ (cf. Figure 6 ).

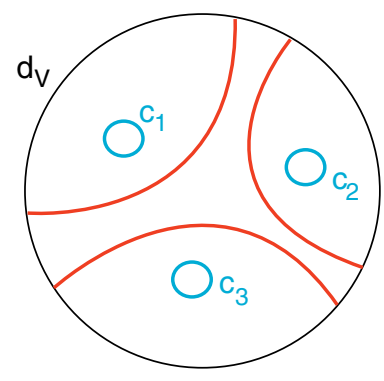

Figure 6 . The maximal possible $D$-edges in $\bar{\Gamma}_{P}$

Lemma 6.2. There can be no $S$-cycle in $\Gamma_{P}$ consisting of D-edges. 
Proof. Suppose otherwise that $\left\{e_{1}, e_{2}\right\}$ is an $S$-cycle in $\Gamma_{P}$ consisting of $D$-edges with label pair $\{j, j+1\}$. We may assume that the bigon face $E$ between $e_{1}$ and $e_{2}$ lies on the $X_{1}$-side of $A$.

Let $H$ be the portion of the filling solid torus of $L(p, 2 q)$ lying in $X_{1}$ which contains $\hat{b}_{j}$ and $\hat{b}_{j+1}$. In $\Gamma_{Q}, e_{1} \cup b_{j} \cup e_{2} \cup b_{j+1}$ cannot be contained in a disk region $D_{*}$ of $A$ as otherwise a regular neighbourhood of $D_{*} \cup E \cup H$ in $X_{1}$ would be a punctured projective space. Thus $e_{1} \cup b_{j} \cup e_{2} \cup b_{j+1}$ contains a core circle of $A$ (cf. Figure (7).

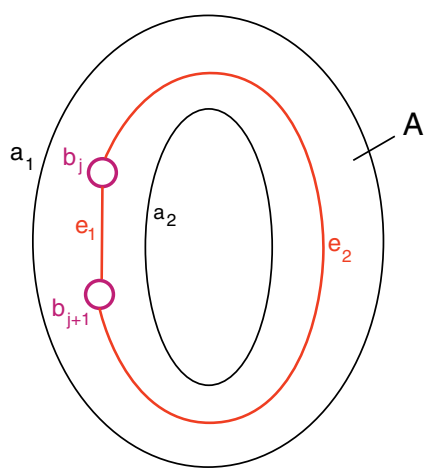

Figure 7. The corresponding cycle $\left\{e_{1}, e_{2}\right\}$ in $\Gamma_{Q}$

Let $U$ be a regular neighbourhood of $E \cup H \cup A$ in $X_{1}$. Then $U$ is a solid torus and the frontier of $U$ in $X_{1}$ is an annulus $\left(A^{\prime}, \partial A^{\prime}\right) \subset\left(X, T_{K}\right)$ for which $\partial A^{\prime}$ is parallel to $\partial A$ in $T_{K}$ and which intersects $T_{V}$ in $n-2$ components. By construction, $A^{\prime}$ is inessential in $X_{1}$ and therefore $X_{1}$ cannot be a torus cross interval. It follows that $X_{1}$ is a fibred solid torus of $X$. Since $A^{\prime}$ has winding number 2 in the solid torus $U$, the singular fibre of $X_{1}$ has order 2, contrary to Lemma 6.1. Thus the lemma holds.

Note that $\Gamma_{P}$ has at most six $C D$-edges and thus $\Gamma_{P}$ has at least $(n p-6) / 2$ $D$-edges, so there is a family of at least $(n p-6) / 6$ mutually parallel $D$-edges. By Lemma 6.2 we have $(n p-6) / 6 \leq n / 2$. Hence $n \leq 6 /(p-3)$, and therefore $p=5$ and $n=2$. If $\Gamma_{P}$ has a $C$-edge, it would have only one family of parallel $D$-edges, and this family would have at least three edges, contrary to the fact that no two $D$-edges can be parallel in $\Gamma_{P}$ by Lemma 6.2. Also, $\Gamma_{P}$ has at least four $C D$-edges, as otherwise there would be four $D$-edges, two of which would form an $S$-cycle. Thus $\Gamma_{P}$ has either six or four $C D$-edges.

We first consider the case when there are exactly four $C D$-edges. In this case we have three $D$-edges in $\Gamma_{P}$, no two of which can be parallel. Hence $\Gamma_{P}$ may be assumed to be as illustrated in Figure 8, i.e. $c_{1}$ and $c_{2}$ are contained in $T_{K}$ and $c_{3}$ is contained $\partial X \backslash T_{K}$. Thus $|L|=|\partial X|=2$, and we may assume that $X_{1}$ is a solid torus and $X_{2}$ is a torus cross interval. In particular, $c_{3}$ is contained in $X_{2}$.

Consider the face $f$ given in Figure 8 . From this figure we see that $f$ and $c_{3}$ are on the same side of $A$ (since $A$ is separating in $X$ ), and thus $f$ is contained in $X_{2}$. Let $T_{*}$ be the component of $\partial X_{2}$ containing $A$, and $H$ that part of the filling solid torus of $L(p, 2 q)$ contained in $X_{2}$. We use $\partial_{0} H$ to denote $\partial H \cap T_{V}$. It is evident that the boundary $\partial f$ of $f$ is contained in $T_{*} \cup \partial_{0} H$. Also note that $\partial f \cap T_{*}$ 


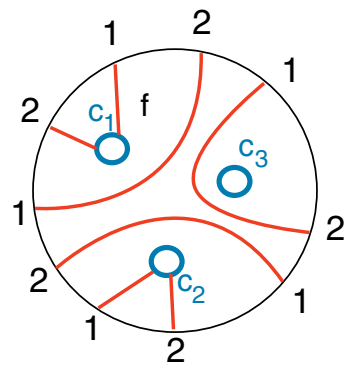

Figure $8 . \Gamma_{P}$ when $\Delta(\alpha, \beta)=5, n=2$ and $4 C D$-edges

cannot be contained in a disk in $T_{*}$, as otherwise $X_{2}$ would contain a projective space as a summand. Thus $\partial f \cap T_{*}$ is contained in an annulus $A_{*}$ of $T_{*}$. A regular neighbourhood $W$ of $H \cup f \cup T_{*}$ in $X_{2}$ is a Seifert fibred space whose base orbifold is an annulus with a cone point of order 2. Since $X_{2}$ is a torus cross interval, the frontier of $W$ in $X_{2}$ is an incompressible torus in $X_{2}$. But this torus cannot be parallel to $T_{*}$ in $X_{2}$, contradicting the fact that $X_{2}$ is a torus cross interval. Thus the case when there are exactly four $C D$-edges does not arise.

We now know that $\Gamma_{P}$ must have six $C D$-edges. Hence there are exactly two $D$-edges in $\Gamma_{P}$ and they are not parallel. It follows that $\Gamma_{P}$ is as illustrated in Figure 9 (1) or (2). (Without loss of generality, we may assume that the labels around $d_{V}$ are as shown in these figures and that the vertices $c_{1}, c_{2}$ and $c_{3}$ are numbered as given there.) Therefore $L=K$ and both $X_{1}$ and $X_{2}$ are solid tori.

We are going to show that part (1) of Figure 9 cannot arise and that in the case of part (2) of Figure 9 the dual graph $\Gamma_{Q}$ may be assumed to be as shown in part (6) of Figure 10.

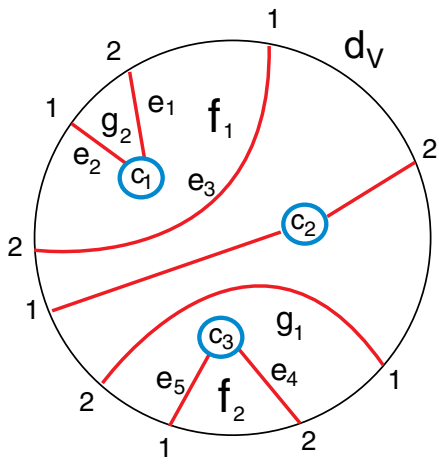

(1)

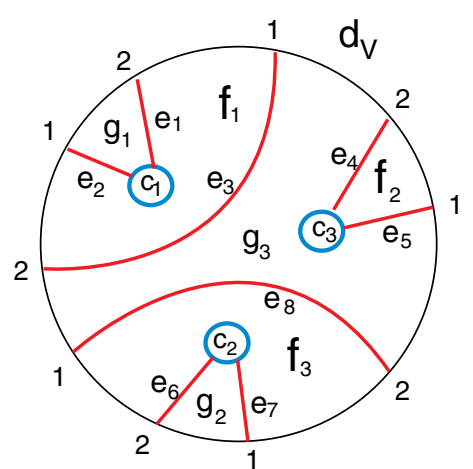

(2)

Figure $9 . \Gamma_{P}$ when $p=5, n=2$ with $6 C D$-edges

Lemma 6.3. The graph $\Gamma_{P}$ cannot be as shown in part (1) of Figure 9 , 
Proof. Suppose otherwise that $\Gamma_{P}$ is given by part (1) of Figure 9. Since $A$ is a separating annulus, the faces $f_{1}, f_{2}$ of $\Gamma_{P}$ lie on the same side of $A$, say in $X_{1}$, and the faces $g_{1}, g_{2}$ lie in $X_{2}$.

Let $H$ be the part of the filling solid torus of $L(p, 2 q)$ contained in $X_{1}$ and set $\partial_{0} H=\partial H \cap T_{V}$. The boundary edges of $f_{1}$ consist of two $C D$-edges $e_{1}, e_{2}$ and one $D$-edge $e_{3}$. Without loss of generality, we may assume that the label of the edge $e_{1}$ at the vertex $c_{1}$ is 2 . In $\Gamma_{Q}$, the boundary edges of $f_{1}$ may be assumed to be as illustrated in part (1) of Figure 10. Note that the boundary $\partial f_{1}$ of $f_{1}$, including the corners, lies in $\partial X_{1} \cup \partial_{0} H$. Further, $\partial f_{1} \cap \partial X_{1}$ is contained in an annulus $A_{*}$ of $\partial X_{1}$ whose slope has distance 1 from that of $\partial A$. Note as well that $\partial f_{1} \cap\left(\partial X_{1} \backslash A\right)$ is an essential arc in the annulus $\left(\partial X_{1} \backslash A\right)$. A regular neighbourhood $U$ of $H \cup f_{1} \cup A_{*}$ in $X_{1}$ is a solid torus whose frontier in $X_{1}$ is an annulus $A_{\#}$ of winding number 2 in $U$. Thus $A_{\#}$ must be parallel to $\partial X_{1} \backslash A_{*}$ through $X_{1} \backslash U$. It follows that the fundamental group of $X_{1}$ is carried by $U$ and thus has presentation

$$
\left\langle x, t: x^{2} t=1\right\rangle
$$

where we take a fat base point in $A$ containing $b_{1} \cup b_{2} \cup\left(\partial f_{1} \cap A\right) \cup$ (all $A B$-edges), $x$ is a based loop formed by a cocore arc of $\partial_{0} H$, and $t$ is a based loop formed by a cocore arc of $\partial X_{1} \backslash A$.

Now consider the face $f_{2}$. We claim that the label of the edge $e_{4}$ at the vertex $c_{3}$ cannot be 2 . Otherwise in $\Gamma_{Q}$, the boundary edges of $f_{2}, e_{4}$ and $e_{5}$ would be as depicted in part (2) or part (3) of Figure 10. In either case, the face $f_{2}$ would add the relation $x t s=1$ to the presentation for $\pi_{1}\left(X_{1}\right)$ above, where $s$ is the element represented by a core circle of the annulus $A$. Thus the fundamental group of the solid torus $X_{1}$ would be generated by $s=x$. But $s$ can be considered as a regular fibre of $X$. So the singular fibre of $X_{1}$ would have order one, which contradicts Lemma 6.1.

Thus the label of $e_{4}$ at $c_{3}$ is 1 . It follows that in $\Gamma_{Q}$, the edges $e_{4}$ and $e_{5}$ are as shown in part (4) of Figure [10, and the face $f_{2}$ adds the relation $x t^{-1} s=1$ to the presentation for $\pi_{1}\left(X_{1}\right)$, where $s$ is the element represented by a core circle of the annulus $A$. Therefore $s=x^{-3}$. Since $s$ can be considered as a regular fibre of $X$ and $x$ can be considered as a core circle of the solid torus $X_{1}$, the singular fibre in $X_{1}$ has order 3 .

By the same argument, we see that the existence of the faces $g_{1}$ and $g_{2}$ in part (1) of Figure 9 implies that the singular fibre in $X_{2}$ has order 3. Hence the two singular fibres of $X$ both have order 3, which implies that the order of the lens space $L(p, 2 q)$ is divisible by 3 . But the lens space has order $p=\Delta(\alpha, \beta)=5$, yielding a contradiction. So part (1) of Figure 9 cannot arise.

So $\Gamma_{P}$ must be as shown in part (2) of Figure 9. Note that the faces $f_{1}, f_{2}, f_{3}$ lie on the same side of $A$, say in $X_{1}$, and the faces $g_{1}, g_{2}, g_{3}$ in $X_{2}$. Arguing similarly as in the proof of Lemma 6.3. we see that in the dual graph $\Gamma_{Q}$ the edges $e_{1}, e_{2}, e_{3}, e_{4}$ and $e_{5}$ may be assumed to be as shown in part (4) of Figure 10.

We now consider the face $g_{3}$. Note that $\partial g_{3}$ must be contained in an annulus $A^{\prime}$ of $\partial X_{2}$ whose slope has distance 1 from that of $\partial A$ and that $\partial g_{3} \cap\left(\partial X_{2} \backslash A\right)$ is an essential arc in the annulus $\left(\partial X_{2} \backslash A\right)$. Thus $e_{8}$ is parallel to $e_{3}$ in $\Gamma_{Q}$. By combining this with the argument given in Lemma 6.3, we see that the graph $\Gamma_{Q}$ must be as depicted in part (5) or part (6) of Figure 10.

Lemma 6.4. Figure 10(5) is impossible. 


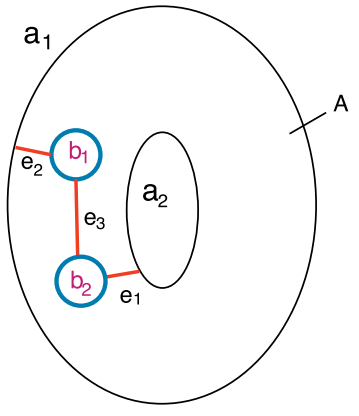

(1)

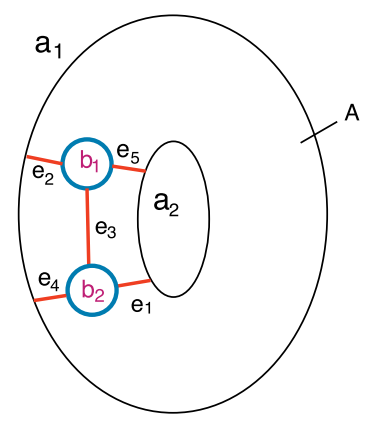

(4)

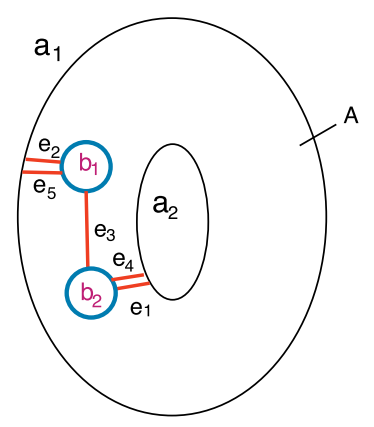

(2)

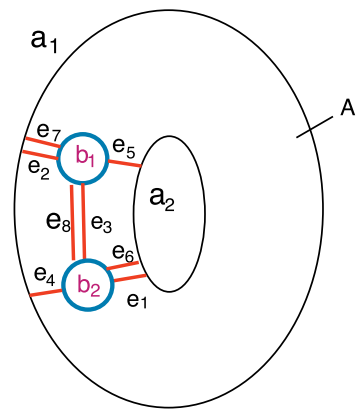

(5)

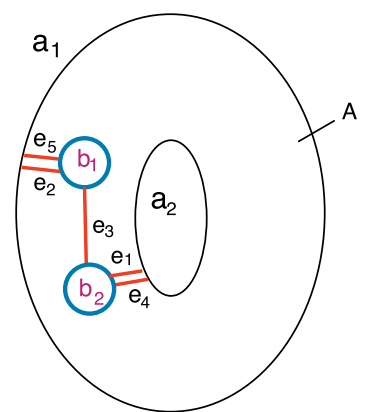

(3)

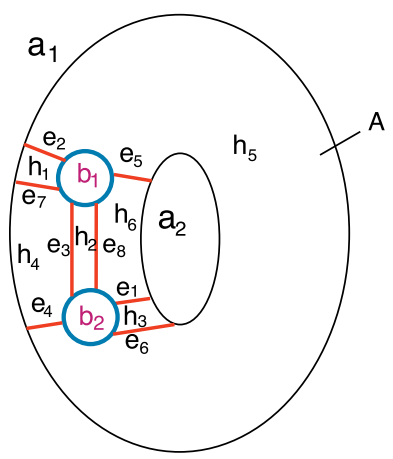

(6)

Figure 10. About the graph $\Gamma_{Q}$

Proof. In Figure 9(2), let $p_{0}, p_{1}, p_{2}, p_{3}, p_{4}$ be the points labeled 1 on $d_{V}$, in cyclic order around $d_{V}$. These are points of intersection of $b_{1}$ with $d_{V}$ on the torus $T_{V}$. It follows that the corresponding points appear around $b_{1}$ in the order $p_{0}, p_{d}, p_{2 d}, p_{3 d}, p_{4 d}$, for some $d$ coprime to $\Delta=\Delta(\alpha, \beta)=5$. The point $p_{i}$ is the endpoint of an edge $e_{j(i)}$. Then, denoting $p_{i}$ by the label $j(i)$ of the corresponding edge, the cyclic order of the $p_{i}$ 's around $d_{V}$ in Figure 9(2) is 28753. In the graph $\Gamma_{Q}$ in Figure 10 (5), the order of the corresponding points is 82753. Since these cyclic orderings are not related in the manner described above, $\Gamma_{Q}$ cannot be as illustrated in Figure $10(5)$.

Remark 6.5. In Figure 10 (6) the order is 27385, which is of the required form, with $d=2$.

So far we have shown that $p=\Delta(\alpha, \beta)=5$ and the graphs $\Gamma_{P}$ and $\Gamma_{Q}$ must be as shown in part (2) of Figure 9 and part (6) of Figure 10, respectively. In the rest of this section we are going to show that these conditions determine the triple $(M, \alpha, \beta)$ uniquely up to homomorphism, and thus it must be the triple $(W h(-3 / 2) ;-5,0)$. 
The surface $Q$ separates $Y$ into $Y_{1}$ and $Y_{2}$, say, where $Y_{i} \subset X_{i}, i=1,2$. Let $N$ be a regular neighbourhood of $T_{V} \cup T_{K} \cup P \cup Q$ in $Y$, and let $\partial_{0} N=\partial N \backslash\left(T_{V} \cup T_{K}\right)$. Then $\partial_{0} N=\partial_{1} N \cup \partial_{2} N_{2}$, where $\partial_{i} N \subset Y_{i}, i=1,2$.

Lemma 6.6. For $i=1$ and $2, \partial_{i} N$ has two components, each a 2-sphere.

Proof. By Remark 6.5, the curves $d_{V}, b_{1}, b_{2}$ on the torus $T_{V}$ are as shown in Figure 11. They decompose $T_{V}$ into rectangles $R_{1}, \ldots, R_{5}, S_{1}, \ldots, S_{5}$, where the $R_{i}$ 's lie in $Y_{1}$ and the $S_{i}$ 's in $Y_{2}$. In Figure 11 a point of intersection of $b_{1} \cup b_{2}$ with $d_{V}$ is labeled with the edge of which it is an endpoint. Similarly, the curves $a_{1}, a_{2}, c_{1}, c_{2}, c_{3}$ decompose the torus $T_{K}$ into rectangles $T_{1}, T_{2}, T_{3}, U_{1}, U_{2}, U_{3}$, where the $T_{j}$ 's lie in $Y_{1}$ and the $U_{j}$ 's in $Y_{2}$. See Figure 12 .

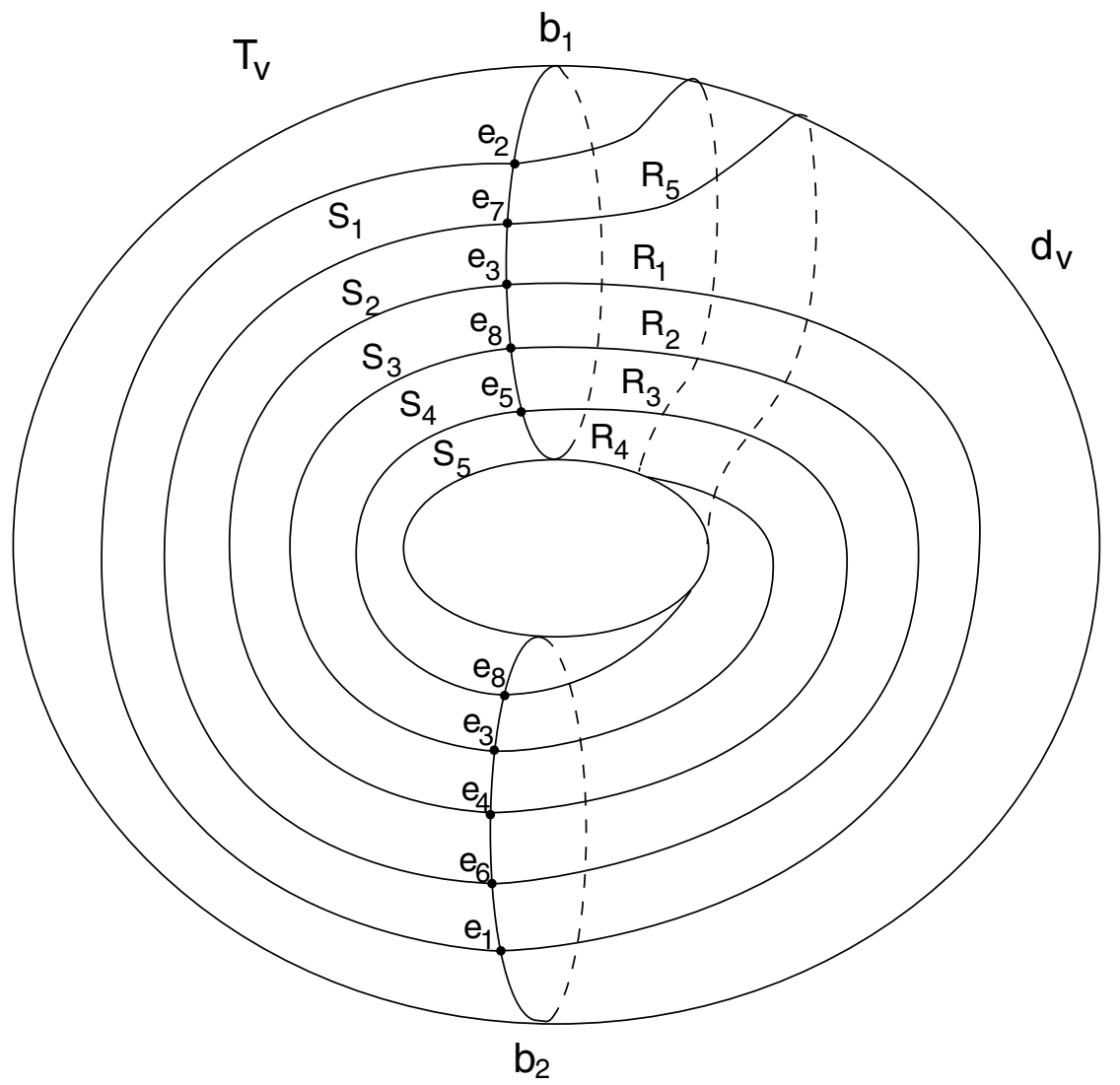

FigURE 11

The faces of the graph $\Gamma_{P}$ are $f_{1}, f_{2}, f_{3}, g_{1}, g_{2}, g_{3}$, where the $f_{i}$ 's lie in $Y_{1}$ and the $g_{i}$ 's lie in $Y_{2}$; see Figure 9(2). Let the faces of $\Gamma_{Q}$ be $h_{1}, \ldots, h_{6}$, as shown in Figure 10(6).

The regular neighbourhood $N$ is the union of product neighbourhoods $T_{V} \times$ $[0,1], T_{K} \times[0,1], P \times[-1,1]$ and $Q \times[-1,1]$, in the obvious way, where $T_{V}=$ $T_{V} \times\{0\}, T_{K}=T_{K} \times\{0\}, P=P \times\{0\}$, and $Q=Q \times\{0\}$. Corresponding to $R_{i}$ is a 2-cell contained in $\left(T_{V} \times\{1\}\right) \cap \partial_{0} N$, which we continue to denote by $R_{i}$; similarly 


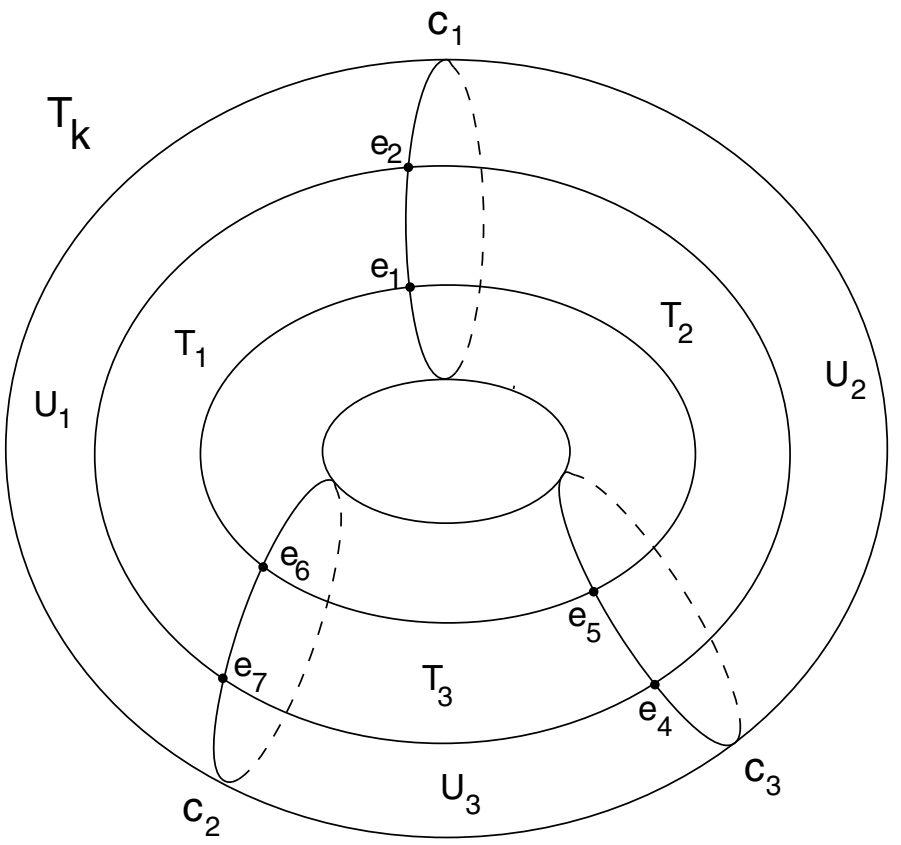

FIGURE 12

for $S_{i}, T_{j}$ and $U_{j}$. A face $f_{i}$ of $\Gamma_{P}$ gives rise to two 2-cells $f_{i}^{+} \subset(P \times\{1\}) \cap \partial_{0} N$ and $f_{i}^{-} \subset(P \times\{-1\}) \cap \partial_{0} N$, and similarly for the $g_{i}$ 's and the faces $h_{k}$ of $\Gamma_{Q}$. Since $h_{k}^{+}$(say) $\subset \partial_{1} N$ and $h_{k}^{-} \subset \partial_{2} N$, there will be no confusion in denoting $h_{k}^{ \pm}$ by $h_{k}$.

By carefully examining the identifications between these various 2-cells one sees that $\partial_{1} N$ has two components $\Sigma_{1}$ and $\Sigma_{1}^{\prime}$, and $\partial_{2} N$ has two components $\Sigma_{2}$ and $\Sigma_{2}^{\prime}$, composed of the following 2-cells:

$$
\begin{aligned}
& \Sigma_{1}: f_{1}^{+}, f_{3}^{-}, h_{1}, h_{2}, h_{3}, R_{2}, R_{5}, T_{1}, \\
& \Sigma_{1}^{\prime}: f_{1}^{-}, f_{2}^{+}, f_{2}^{-}, f_{3}^{+}, h_{4}, h_{5}, h_{6}, R_{1}, R_{3}, R_{4}, T_{2}, T_{3}, \\
& \Sigma_{2}: g_{1}^{+}, g_{2}^{-}, h_{1}, h_{3}, S_{1}, U_{1}, \\
& \Sigma_{2}^{\prime}: g_{1}^{-}, g_{2}^{+}, g_{3}^{+}, g_{3}^{-}, h_{2}, h_{4}, h_{5}, h_{6}, S_{5}, S_{2}, S_{3}, S_{4}, U_{2}, U_{3} .
\end{aligned}
$$

The precise patterns of identification are shown in Figures 13, 14, 15 and 16. respectively. In particular, $\Sigma_{1}, \Sigma_{1}^{\prime}, \Sigma_{2}, \Sigma_{2}^{\prime}$ are 2 -spheres.

Remark 6.7. One can see that $\Sigma_{1}, \Sigma_{1}^{\prime}, \Sigma_{2}, \Sigma_{2}^{\prime}$ are 2 -spheres without completely determining the identification patterns of their constituent 2-cells, by means of the following Euler characteristic computation.

First note that

$$
\chi(P \cup Q)=\chi(P)+\chi(Q)-\chi(P \cap Q)=(-2)+(-2)-8=-12 .
$$

Also, $(P \cup Q) \cap T_{V}$ consists of three circles, meeting in a total number of 10 points. So $\chi\left((P \cup Q) \cap T_{V}\right)=-10$. Similarly, $\chi\left((P \cup Q) \cap T_{K}\right)=-6$. Therefore

$$
\chi(N)=\chi\left((P \cup Q) \cup\left(T_{V} \cup T_{K}\right)\right)=(-12)+0-((-10)+(-6))=4 .
$$

Hence $\chi(\partial N)=8$. 


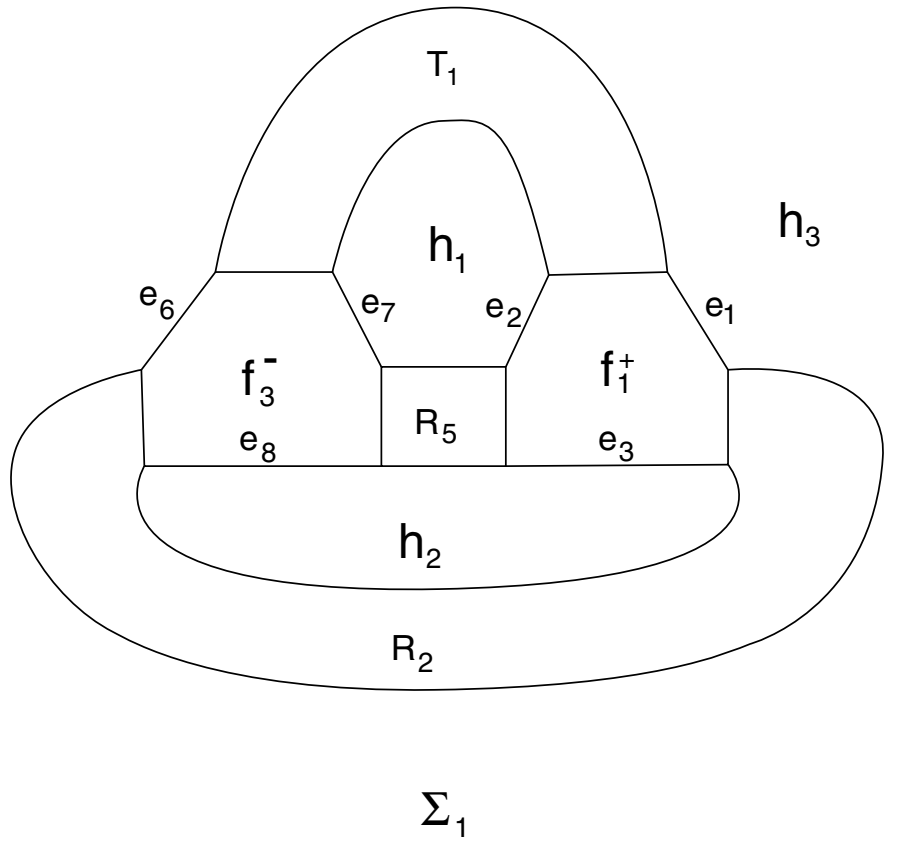

FiguRE 13

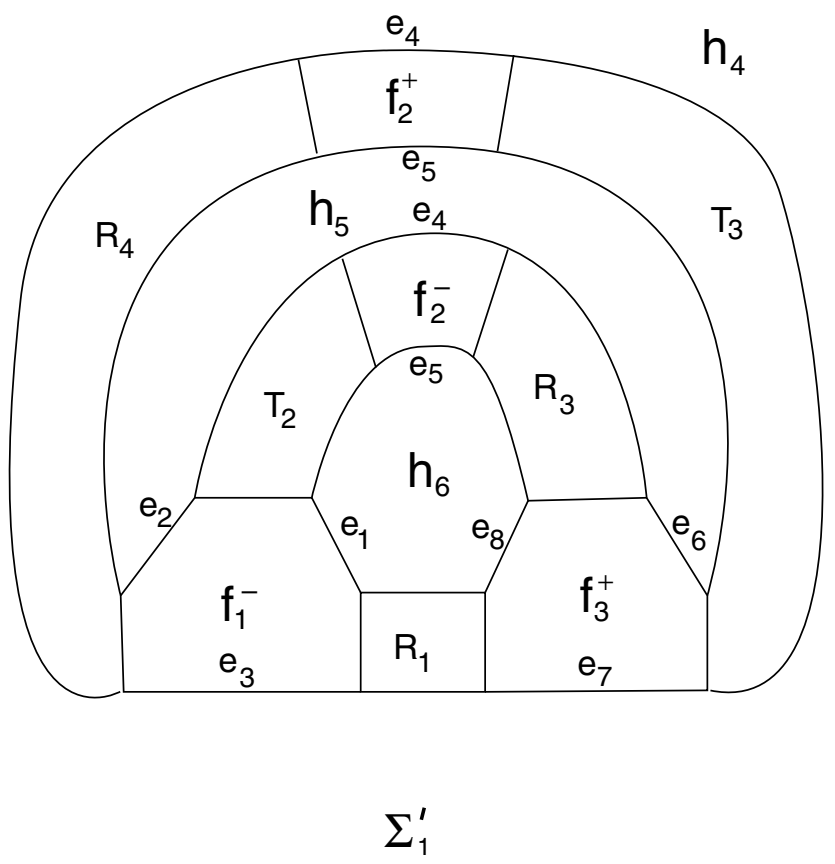

FiguRE 14 


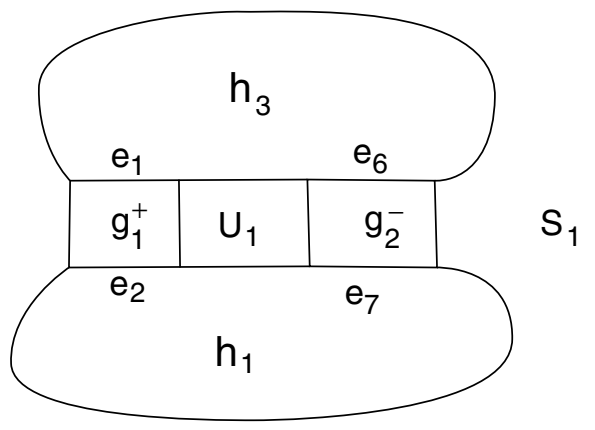

$\Sigma_{2}$

Figure 15

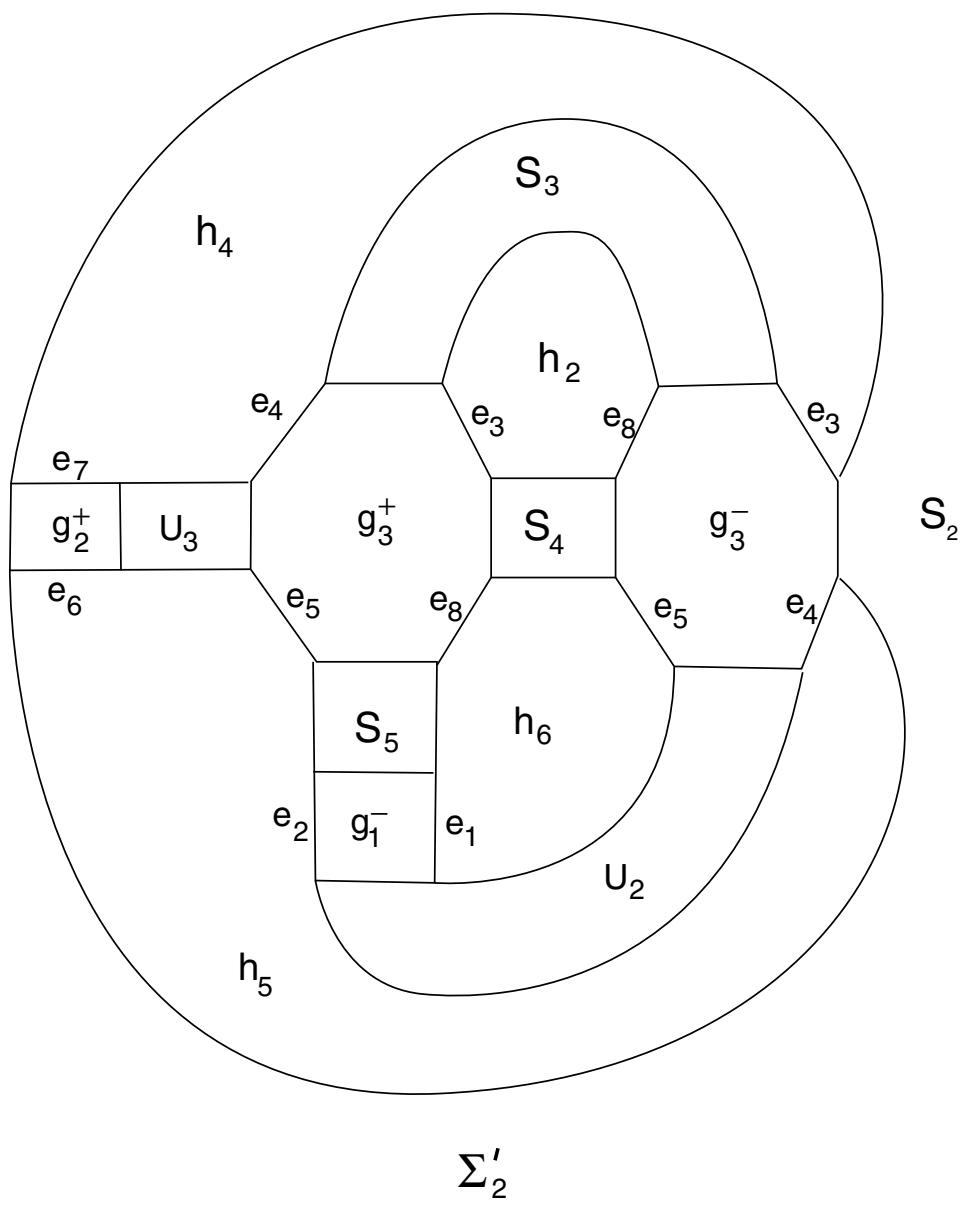

FIGURE 16 
Now one can easily check that each of $\partial_{1} N$ and $\partial_{2} N$ has at most two components. Hence each must have exactly two components, both 2 -spheres.

Proof that $(M ; \alpha, \beta)$ is homeomorphic to $(W h(-3 / 2) ;-5,0)$. Since $Y$ is irreducible the components of $\partial_{0} N$ bound 3-balls in $Y$. Hence the triple $(Y ; P, Q)$ is uniquely determined up to homeomorphism, by Figures 9(2) and 10(6). Since the curves $c_{j}$ are meridians of $L$, the pair $(V, L)$, together with the slopes $\bar{\alpha}, \bar{\beta}$, is uniquely determined. Passing to the double branched cover, we have that $(M ; \alpha, \beta)$ is uniquely determined.

In [MP, Table A3] it is shown that -5 -filling on the hyperbolic manifold $W h(-3 / 2)$ is Seifert fibred with base orbifold $S^{2}(2,3,3)$, while 0 -filling gives a manifold containing a non-separating torus. In fact, it is easy to see that $W h(-3 / 2)$ contains an essential once-punctured torus with boundary slope 0 . Hence $(M ; \alpha, \beta) \cong$ $(W h(-3 / 2) ;-5,0)$.

\section{The CASE $\Delta(\alpha, \beta)=7$ AND The INVOLUtion $\tau_{\alpha}$ REVERSES THE ORIEnTATIONS OF THE SEIFERT FIBREs OF $M(\alpha)$}

In this section we suppose that Assumptions 5.1 hold and show that it is impossible for $\Delta(\alpha, \beta)$ to be 7 and for $\tau_{\alpha}$ to reverse the orientations of the Seifert fibres of $M(\alpha)$. We assume otherwise in order to obtain a contradiction.

A tangle will be a pair $\mathcal{T}=(R, t)$, where $R$ is $S^{3}$ minus the interiors of a disjoint union of 3-balls and $t$ is a properly embedded 1 -manifold. Let $\widetilde{\mathcal{T}}=(X, \tilde{t})$ be the double branched cover of $\mathcal{T}$. In our examples each boundary component $S$ of $R$ will meet $t$ in either 4 or 6 points, and hence the corresponding boundary component $\widetilde{S}$ of $X$ is either a torus or a surface of genus 2 , respectively.

An essential disk in $\mathcal{T}$ is a properly embedded disk $D$ in $R$ such that either

(i) $D \cap t=\emptyset$ and $\partial D$ does not bound a disk in $\partial R \backslash t$, or

(ii) $D$ meets $t$ transversely in a single point and $\partial D$ does not bound a disk in $\partial R$ containing a single point of $t$.

It follows from the $\mathbb{Z} / 2$-equivariant Disk Theorem ([GLi], [KT, YYM]) that $X$ contains an essential disk $\widetilde{D}$, i.e., a properly embedded disk such that $\partial \widetilde{D}$ is essential in $\partial X$, if and only if $\mathcal{T}$ contains an essential disk $D$.

If $S$ is a boundary component of $R$ such that $|S \cap t|=4$, a marking of $S$ is a specific identification of $(S, S \cap t)$ with $\left(S^{2},\{N E, N W, S W, S E\}\right)$. We can then attach a rational tangle $\mathcal{R}(\gamma)$ to $\mathcal{T}$ along $S$ with respect to this marking, where $\gamma \in \mathbb{Q} \cup\{1 / 0\}$

By Lemma 4.4 (1), $M(\alpha)=M(7 / q)$ has base orbifold $S^{2}(7,7, m)$ for some odd integer $m \geq 3$. As in Lemma 5.3 , let $\widetilde{M}_{7}$ be the 7 -fold cyclic cover of $M$. Then $\partial \widetilde{M}_{7}$ is a single torus, and both $\alpha$ and $\beta$ lift to slopes $\tilde{\alpha}$ and $\tilde{\beta}$ in $\partial \widetilde{M}_{7}$, i.e. $\widetilde{M}_{7}(\tilde{\alpha})$ is a 7-fold cyclic cover of $M(\alpha)$ and $\widetilde{M}_{7}(\tilde{\beta})$ is a 7-fold cyclic cover of $M(\beta)$. Furthermore the involution $\tau$ on $M$ lifts to an involution $\tilde{\tau}$ on $\widetilde{M}_{7}$ and $\tilde{V}=\widetilde{M}_{7} / \tilde{\tau}$ is a 7 -fold cyclic cover of $M / \tau=V$. So $\tilde{V}$ is a solid torus. The involution $\tilde{\tau}$ extends to an involution $\tilde{\tau}_{\tilde{\alpha}}$ on $\widetilde{M}_{7}(\tilde{\alpha})$ such that $\widetilde{M}_{7}(\tilde{\alpha}) / \tilde{\tau}_{\tilde{\alpha}}=S^{3}$ is the 7 -fold cyclic cover of the lens space $M(\alpha) / \tau_{\alpha}=L(7,2 q)$. Let $L_{7}$ be the inverse image of $L$ in $S^{3}$. Then by Lemma [5.9, $L_{7}$ is as shown in Figure 17, where the box with an integer $r$ in it stands for $r$ full horizontal twists, and by Lemma 4.4(2), $L_{7}$ is also as shown in Figure 18, where the box with an integer $r^{\prime}$ in it stands for $r^{\prime}$ full horizontal twists. 
Since $p=7, n$ is odd by Lemma $5.2(3)$. Hence from Figure 18 we see that $L_{7}$ is a single knot. So to get a contradiction, we just need to show that the two knots $K$ and $K^{\prime}$ shown in Figures 17 and 18, respectively, are inequivalent.

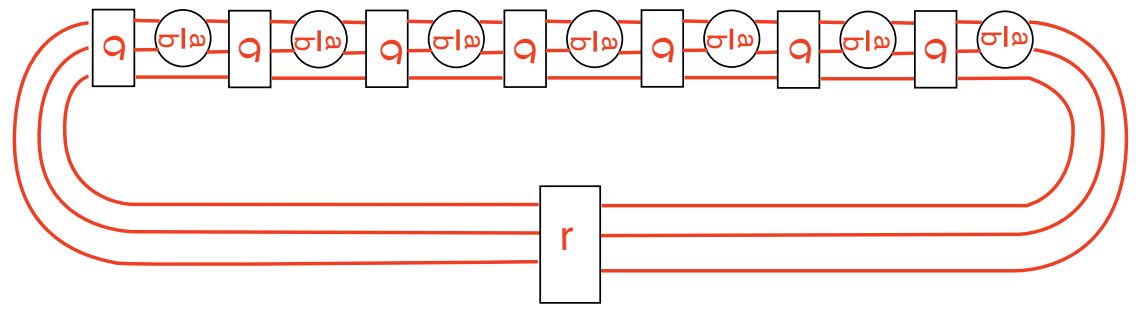

FigURE 17

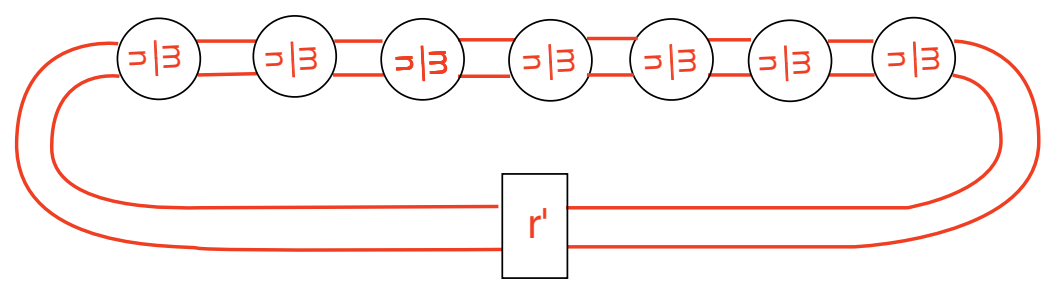

FiguRE 18

Theorem 7.1. The knots $K$ and $K^{\prime}$ are inequivalent.

Let $W, W^{\prime}$ be the double cover of $S^{3}$ branched over $K, K^{\prime}$, respectively. We shall show that $W$ and $W^{\prime}$ are not homeomorphic. Note that $W^{\prime}$ is a Seifert fibred manifold with base orbifold $S^{2}(m, m, m, m, m, m, m)$. We will examine $W$ and show that it cannot be such a Seifert manifold.

Let $\mathcal{T}=(R, t)$ be the tangle shown in Figure 19, Let the boundary components of $R$ be $S, S_{1}, S_{2}, S_{3}$ as shown. Note that $|t \cap S|=6$ and $\left|t \cap S_{i}\right|=4, i=1,2,3$. Let $X$ be the double branched cover of $\mathcal{T}$. Then $\partial X=G \amalg \coprod_{i=1}^{3} T_{i}$, where $G$ is the double branched cover of $(S, S \cap t)$ and $T_{i}$ is the double branched cover of $\left(S_{i}, S_{i} \cap t\right), i=1,2,3$; thus $G$ has genus 2 and the $T_{i}$ are tori.

Remark 7.2. The permutation induced by $\sigma$ takes 1 to 2 or 3 , since $K$ is connected.

Proposition 7.3. $X(a / b, a / b, a / b)$ is either

(1) boundary-irreducible, or

(2) the boundary connected sum of two copies of a Seifert fibred manifold with base orbifold $D^{2}(a, d), d>1$, or

(3) a handlebody of genus 2.

We prove Proposition 7.3 by successively filling along $T_{1}, T_{3}$ and $T_{2}$.

Lemma 7.4. $G$ is incompressible in $X$.

Proof. Because of Remark 7.2 above, the arrangement of the components of $t$ with respect to the boundary components of $R$ is as illustrated schematically in Figure 20. It follows easily that $\mathcal{T}=(R, t)$ cannot contain any essential disk $D$ with $\partial D \subset S$. 


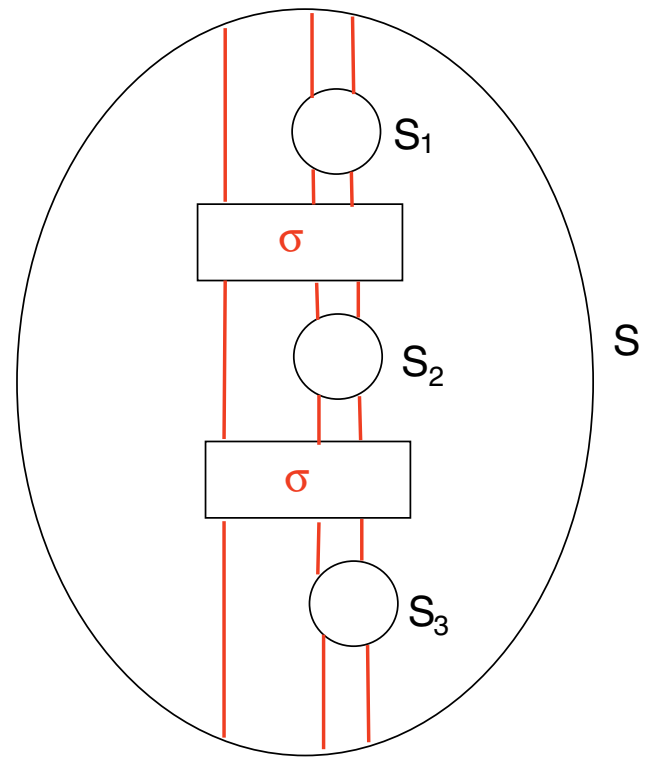

FIGURE 19

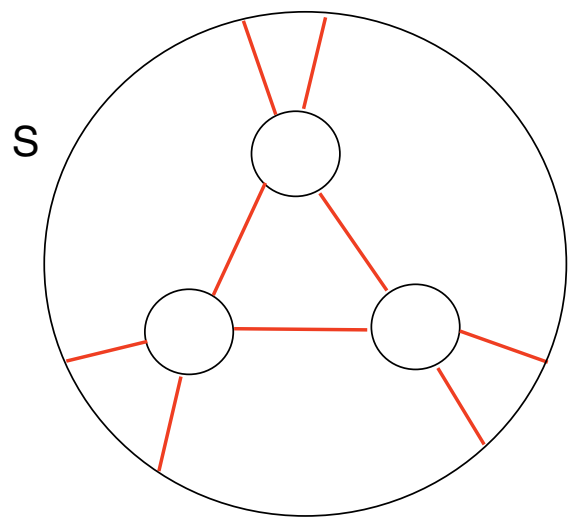

FIGURE 20

In the sequel, a " $*$ " will indicate that the corresponding boundary component is left unfilled.

Lemma 7.5. $G$ is incompressible in $X(a / b, *, *)$.

Proof. There is an essential annulus $A_{1} \subset R$, disjoint from $t$, with one boundary component in $S$ and the other having slope $0 / 1$ on $S_{1}$; see Figure 21. A component of the inverse image of $A_{1}$ in $X$ is an essential annulus with one boundary component on $G$ and the other having slope $0 / 1$ on $T_{1}$. Since $\Delta(a / b, 0 / 1)=a>1$, it follows from [Sh] and Lemma 7.4 that $G$ is incompressible in $X(a / b, *, *)$.

Lemma 7.6. $G$ is incompressible in $X(a / b, *, a / b)$. 


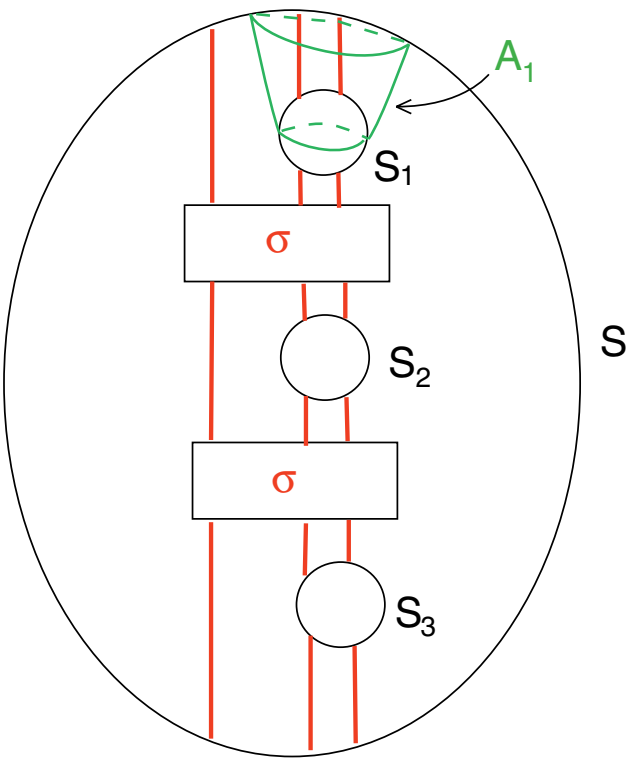

FiguRE 21

Proof. There is an essential annulus $A_{3} \subset R(a / b, *, *)$ with one boundary component on $S$ and the other having slope $0 / 1$ on $S_{3}$. The result now follows as in the proof of the previous lemma.

Proof of Proposition 7.3 . There is an essential disk in $\mathcal{T}(a / b, 0 / 1, a / b)$, meeting $t(a / b, 0 / 1, a / b)$ in a single point; see Figure 22. Therefore $G$ is compressible in $X(a / b, 0 / 1, a / b)$. Since $\Delta(a / b, 0 / 1)=a>1$, it follows from Lemma 7.6 and Wu2 that either $G$ is incompressible in $X(a / b, a / b, a / b)$ or there is an essential annulus $A \subset X(a / b, *, a / b)$ with one boundary component on $G$ and the other having slope $r / s$ on $T_{2}$, where $\Delta(r / s, 0 / 1)=\Delta(r / s, a / b)=1$. We may assume the latter, in which case, by Dehn twisting $X(a / b, *, a / b)$ along $A$, we have that $X(a / b, a / b, a / b) \cong$ $X(a / b, 0 / 1, a / b)$. From Figure 22 we see that $X(a / b, 0 / 1, a / b)$ is the boundary connected sum of two copies of $Y$, the double branched cover of the tangle shown in Figure 23,

The disk $D$ shown in Figure 23 separates the tangle into two rational tangles $\mathcal{R}, \mathcal{R}^{\prime}$ and lifts to an annulus $A \subset Y$ which separates $Y$ into two solid tori $U$ and $U^{\prime}$, the double branched covers of $\mathcal{R}, \mathcal{R}^{\prime}$, respectively. Note that $A$ has a winding number $a$ in $U$. Also, it is easy to see (by Remark 7.2) that $A$ is not meridional on $U^{\prime}$. Hence $Y$ is either a Seifert fibre space with base orbifold $D^{2}(a, d)$, for some $d>1$, or a solid torus, giving conclusions (2) and (3), respectively.

Let $Z$ be the double branched cover of the tangle $(Q, s)$ shown in Figure 24 . Then $\partial Z$ has one torus component and two genus two components.

Lemma 7.7. $Z(a / b)$ has incompressible boundary.

Proof. For $i=0,1$, there is an annulus $A_{i} \subset Q$, disjoint from $s$, with one boundary component on $S_{i}$ and the other having slope $0 / 1$ on $S$, as shown in Figure 24. Since $\Delta(a / b, 0 / 1)=a>1$, the result follows as in the proof of Lemma 7.5 


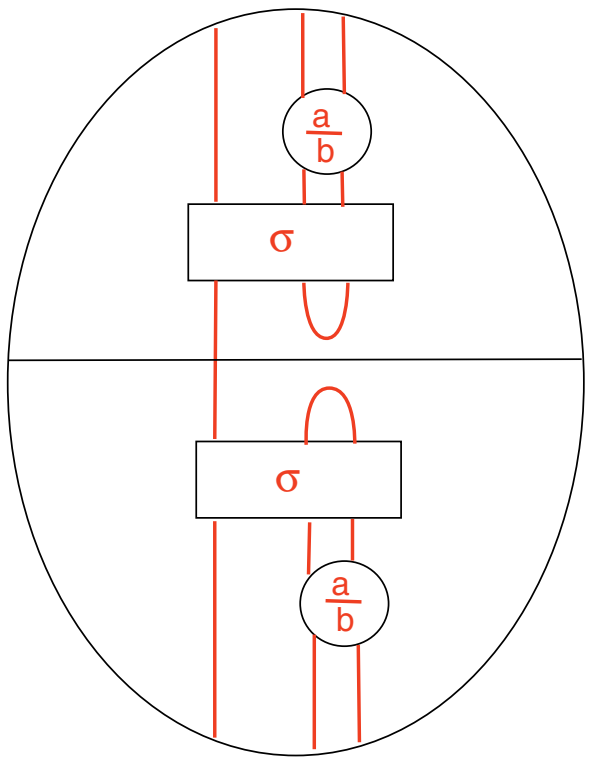

FIGURE 22

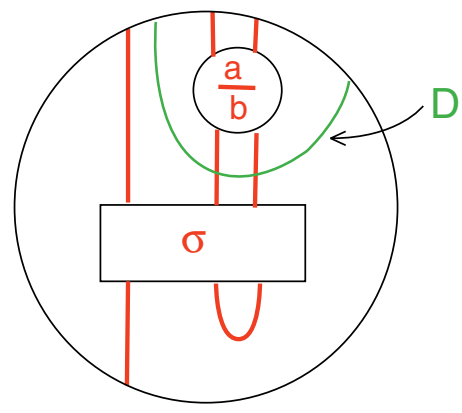

FIGURE 23

Note that filling $(Q, s)$ along $S$ with the rational tangle $\mathcal{R}(1 / 0)$ gives a product tangle. Hence $Z \cong G \times I-\operatorname{int} N(C)$, where $G$ is a surface of genus two and $C$ is a simple closed curve $\subset G \times\{1 / 2\}$.

Proposition 7.8. The double branched cover $W$ of $\left(S^{3}, K\right)$ either

(1) contains a separating incompressible surface of genus 2 , or

(2) contains four disjoint tori, each cutting off a manifold which is Seifert fibred over $D^{2}(a, d), d>1$, or

(3) has Heegaard genus at most 3.

Proof. From Figure 25 we see that $W \cong P \cup_{G} Z(a / b) \cup_{G^{\prime}} P^{\prime}$, where $P$ and $P^{\prime}$ are copies of $X(a / b, a / b, a / b)$.

Case (1) of Proposition 7.3, together with Lemma 7.7, gives conclusion (1). 


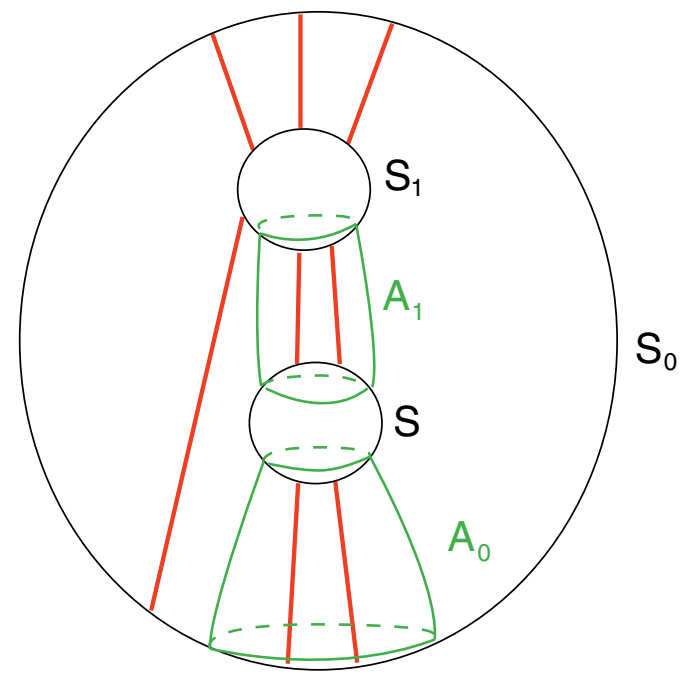

FiguRE 24

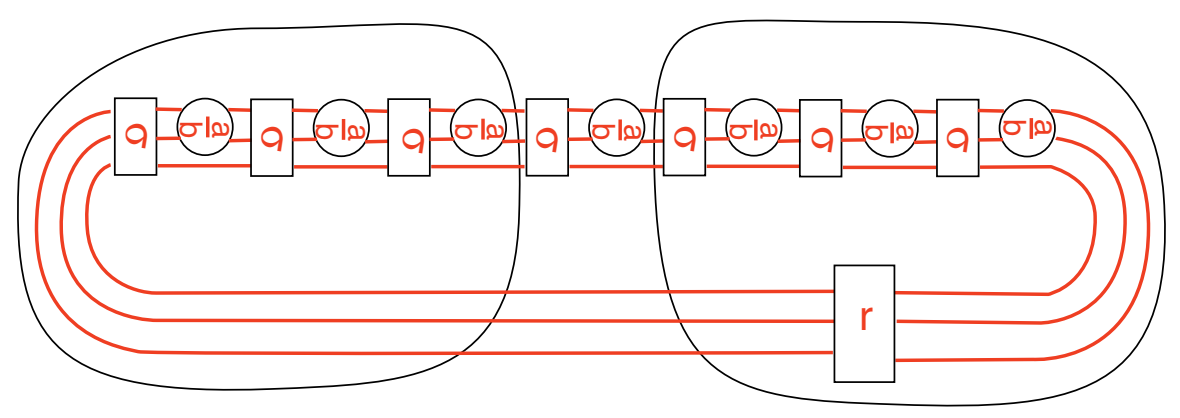

FiguRE 25

In Case (2) of Proposition [7.3, each of $P, P^{\prime}$ contains two disjoint tori, each cutting off a manifold which is Seifert fibred over $D^{2}(a, d)$, and we have conclusion (2).

In Case (3) of Proposition 7.3. $P$ and $P^{\prime}$ are handlebodies of genus 2. Also, by the remark after the proof of Lemma 7.7 $Z(a / b)$ is obtained from $G \times I$ by Dehn surgery on a curve in $G \times\{1 / 2\}$. Hence $W$ is obtained from a closed manifold with a Heegaard splitting of genus 2 by a Dehn surgery on a curve in the Heegaard surface. Since such a curve has tunnel number at most 2, $W$ has Heegaard genus at most 3 .

Proof of Theorem 7.1. To get a contradiction, suppose $W \cong W^{\prime}$.

Recall that $W^{\prime}$ is the double branched cover of $\left(S^{3}, K^{\prime}\right)$ and is a Seifert fibred space with base orbifold $S^{2}(m, m, m, m, m, m, m)$.

In Case (1) of Proposition 7.8, $W^{\prime}$ would contain a separating incompressible surface of genus 2. This surface would have to be horizontal, and would then separate $W^{\prime}$ into two twisted $I$-bundles. Thus $W^{\prime}$ would contain a non-orientable 
surface. But since $W^{\prime}$ is the double branched cover of a knot in $S^{3}, H_{1}\left(W^{\prime} ; \mathbb{Z} / 2\right)=$ 0 , a contradiction.

In Case (2) of Proposition 7.8, the tori in question are incompressible (otherwise $W^{\prime}$ would have base orbifold $S^{2}(a, d, r)$ for some $\left.r \geq 1\right)$. Hence they are vertical in $W^{\prime}$. But since $W^{\prime}$ has only 7 exceptional fibres, this is clearly impossible.

Finally, since $W^{\prime}$ has base orbifold $S^{2}(m, m, m, m, m, m, m)$, every irreducible Heegaard splitting of $W^{\prime}$ is either horizontal or vertical by [MSch]. It also follows from MSch that when $W^{\prime}$ has an irreducible horizontal Heegaard splitting, its genus is larger than 6 , and that any irreducible vertical Heegaard splitting of $W^{\prime}$ has genus 6. Hence Case (3) of Proposition 7.8 is impossible.

\section{The CASe $\Delta(\alpha, \beta)=5$ And $M(\alpha)$ IS A LENS SPACE}

In this section we suppose that Assumptions 5.1 hold and show that $M(\alpha)$ cannot be a lens space, thus completing our proof of Baker's theorem Ba. As we noted at the end of $\$ 5$, it suffices to show that the link depicted in Figure 3 , considered as lying in a Heegaard solid torus in $L(5,2 q)$, is not isotopic to either the core of a Heegaard solid torus or the boundary of a Möbius band spine of a Heegaard solid torus.

The proof of the following lemma is straightforward.

Lemma 8.1. Let $V_{1}$ be a Heegaard solid torus in a lens space $L(p, q)$ and let $K$ be either a core of $V_{1}$ or a $(2, k)$-cable of a core of $V_{1}$. In the first case assume that $p$ is odd. Then the double branched cover of $(L(p, q), K)$ is a lens space.

Remark 8.2. The condition that $p$ be odd in the first case is needed to guarantee the existence of a double branched cover. Furthermore, in that case we have $L(p, q) \cong$ $L(p, 2 r) \cong L\left(p, 2 r^{\prime}\right)$, where $4 r r^{\prime} \equiv 1(\bmod p)$, and then the double branched cover is homeomorphic to either $L(p, r)$ or $L\left(p, r^{\prime}\right)$.

Lemma 8.3. Let $Q$ be a once-punctured torus bundle over $S^{1}$, with $\beta$ the boundary slope of the fibre, and let $\gamma$ be a slope on $\partial Q$ such that $Q(\gamma)$ is reducible. Then $\Delta(\beta, \gamma)=1,2,3,4$ or 6 .

Proof. We consider separately three possibilities for $Q$.

(1) $Q$ is hyperbolic. Here $\Delta(\beta, \gamma)=1$ by [BZ1, Lemma 4.1].

(2) $Q$ is Seifert fibred. In this case the monodromy of the bundle has finite order, $d$, say, where $d=1,2,3,4$ or 6 . If $Q(\gamma)$ is reducible, then $\gamma$ is the Seifert fibre slope, and hence $\Delta(\beta, \gamma)=d$.

(3) $Q$ is toroidal and not Seifert fibred. Let $T_{0}$ be the once-punctured torus fibre of $Q$. Here the monodromy of the bundle is \pm the $r^{\text {th }}$ power of a Dehn twist along an essential loop $x$ in $T_{0}$, where $r \neq 0$ and $+/-$ denotes composition with the identity and the elliptic involution, respectively. The free group $\pi_{1}\left(T_{0}\right)$ has basis $\{x, y\}$ with $\left[\partial T_{0}\right]=[x, y]=x y x^{-1} y^{-1}$. Then $\pi_{1}(Q)$ has presentation

$$
\left\langle x, y, t: t^{-1} x t=x, t^{-1} y t=y x^{r}\right\rangle
$$

or

$$
\left\langle x, y, t: t^{-1} x t=(x y) x^{-1}(x y)^{-1}, t^{-1} y t=x\left(x^{-r} y^{-1}\right) x^{-1}\right\rangle
$$

in the $+/-$ cases mentioned above. In both cases $\pi_{1}(\partial Q)=\langle t,[x, y]\rangle$.

For the proof in this case we will use the following lemma. 
Lemma 8.4. If $A * B$ is a non-trivial free product quotient of $\pi_{1}(Q)$, then $4 t=$ $0 \in H_{1}(A * B)$.

Proof. Let $A * B$ be a quotient of $\pi_{1}(Q)$ with $A \neq 1 \neq B$. We adopt the convention that a word in $x, y$ and $t$ denotes the image in $A * B$ of the corresponding element of $\pi_{1}(Q)$.

Case (i). Here $x$ and $t$ commute. Hence either

(a) $x$ and $t$ are powers of some element $z$, or

(b) $x$ and $t$ lie in a conjugate of a factor.

In subcase (a) we have $x=z^{m}, t=z^{n}$, say. The second relation in the presentation (i) gives $z^{-n} y z^{n}=y z^{r m}$, and therefore $y^{-1} z^{n} y=z^{n-r m}$. By applying an inner automorphism of $A * B$ we may assume that $z$ is represented by a cyclically reduced word in the factors. It follows that $|n|=|n-r m|$, otherwise we have two cyclically reduced words, $z^{n}$ and $z^{n-r m}$, of different lengths in the same conjugacy class. Hence either $m=0$ or $y^{-1} z^{n} y=z^{-n}$. If $m=0$ then $x=1$, and so $A * B$ is a quotient of $\left\langle y, t: t^{-1} y t=y\right\rangle \cong \mathbb{Z} \times \mathbb{Z}$, a contradiction. If $y^{-1} z^{n} y=z^{-n}$ then $y^{-1} t y=t^{-1}$, and so $2 t=0 \in H_{1}(A * B)$.

In subcase (b) we may assume, by applying an inner automorphism of $A * B$, that $x, t \in A$. Then $y^{-1} t^{-1} y=x^{r} t^{-1} \in A$. But $t^{-1} \in A$, and hence $y \in A$. Therefore $B=1$, a contradiction.

Case (ii). Let $s=t x y$. Then $\pi_{1}(Q)$ has the presentation

$$
\left\langle x, y, s: s^{-1} x s=x^{-1}, s^{-1} y s=y^{-1} x^{-r}\right\rangle .
$$

Since $x$ and $s^{2}$ commute, either

(a) $x$ and $s^{2}$ are powers of some element $z$, or

(b) $x$ and $s^{2}$ lie in a conjugate of a factor.

In subcase (a), suppose $x=z^{m}, s^{2}=z^{n}$. The second relation in the presentation of $\pi_{1}(Q)$ implies $s^{-2} y s^{2}=x^{r} y x^{r}$, i.e. $z^{-n} y z^{n}=z^{r m} y z^{r m}$, giving $y^{-1} z^{(n+r m)} y=$ $z^{n-r m}$. As in Case (i) we may assume that $z$ is cyclically reduced, and hence $|n+r m|=|n-r m|$, i.e. either $m=0$ or $n=0$. If $m=0$ then $x=1$, and so $A * B$ is a quotient of the Klein bottle group $\left\langle y, s: s^{-1} y s=y^{-1}\right\rangle$, which is easily seen to imply $A * B \cong \mathbb{Z}_{2} * \mathbb{Z}_{2}$. If $n=0$ then $s^{2}=1$. Hence $2 s=0 \in H_{1}(A * B)$. But in $H_{1}(Q), s=t+x+y, 2 x=0$, and $4 y=0$. Therefore $4 t=0 \in H_{1}(A * B)$.

In subcase (b) we may assume that $x, s^{2} \in A$. Hence $s \in A$. From the second relation in the above presentation of $\pi_{1}(Q)$ we get $\left(y s^{-1}\right)^{2}=x^{-r} s^{-2} \in A$. Therefore $y s^{-1} \in A$, and hence $y \in A$. This implies that $B=1$, a contradiction.

We now complete the proof of Lemma 8.3

Let $\Delta=\Delta(\beta, \gamma)$. Then $\pi_{1}(Q(\gamma))$ is obtained from $\pi_{1}(Q)$ by adding the relation $t^{\Delta}[x, y]^{q}=1$, for some integer $q$ coprime to $\Delta$. It is easy to see from the presentations (i) and (ii) that $H_{1}(Q(\gamma)) \neq \mathbb{Z}$. Therefore $Q(\gamma)$ is a non-trivial connected sum and hence $\pi_{1}(Q(\gamma))$ is a non-trivial free product. The relation $t^{\Delta}[x, y]^{q}=1$ shows that $t$ has order $\Delta$ in $H_{1}(Q(\gamma))$. Hence by Lemma 8.4, $\Delta$ divides 4 .

Now we complete the proof that $M(\alpha)$ cannot be a lens space under the assumption that the conditions [5.1] hold. Suppose otherwise. By Lemma [5.10, $M(\alpha) / \tau_{\alpha} \cong L(5,2 q), L$ is either the core of a Heegaard solid torus in $L(5,2 q)$ or a $(2, k)$-cable of such a core, and furthermore $L(5,2 q)$ has a genus 1 Heegaard splitting $V \cup V_{0}$ such that $L$ is isotopic to a curve in $V$ of the form shown in Figure 
3 where $a$ and $b$ are coprime integers with $a \geq 2$ and $\sigma$ is a 3-braid. We will show that these conditions on $L$ lead to a contradiction.

Remove from the solid torus $V$ in Figure 3 the interior of the 3-ball $B$ containing the $a / b$-rational tangle. We then get a tangle $\mathcal{T}$ in $Y=(V-$ int $B) \cup V_{0}=$ $L(5,2 q) \backslash$ int $B$. Let $X$ be the double branched cover of $(Y, \mathcal{T})$.

Since $\mathcal{T}(a / b)=L$, by Lemma 8.1 we have

- $X(a / b)$ is a lens space.

Also, clearly $\mathcal{T}(0 / 1)=($ core of $V) \#\left(\operatorname{knot}\right.$ in $\left.S^{3}\right)$, so

- $X(0 / 1) \cong L(5, r) \# N$ for some closed 3-manifold $N$.

Lemma 8.5. $X(1 / k)$ is irreducible for all $k \in \mathbb{Z}$.

Proof. $\mathcal{T}(1 / k)$ is (the 3 -braid $\sigma_{1}^{k} \sigma$ in $\left.V\right) \cup V_{0}$. Hence $X(1 / k)=Q_{k} \cup \widetilde{V}_{0}$, where $Q_{k}$ is the double branched cover of $\left(V, \sigma_{1}^{k} \sigma\right)$ and $\widetilde{V}_{0}$ is a solid torus. Now $Q_{k}$ is a $T_{0}$-bundle over $S^{1}$, where $T_{0}$ is the double branched cover of $\left(D^{2}, 3\right.$ points), i.e. a once-punctured torus. Let $\beta$ be the boundary slope of the fibre of $Q_{k}$; note that $\beta$ projects to the meridian $\mu$ of $V$. Let $\mu_{0}, \tilde{\mu}_{0}$ be the meridians of $V_{0}, \widetilde{V}_{0}$, respectively. Since $\Delta\left(\mu, \mu_{0}\right)=5$, we have $\Delta\left(\beta, \tilde{\mu}_{0}\right)=5$. Hence by Lemma 8.3, $X(1 / k)$ is irreducible.

There is a $\mathbb{Z} / 2$-action on $X$ with quotient $Y=L(5,2 q) \backslash$ int $B$. It follows easily that $X$ is not a solid torus. We consider the following three possibilities for $X$.

(1) $X$ is reducible. Here we must have $X \cong X^{\prime} \# X(a / b)$, where $X^{\prime}(a / b) \cong S^{3}$. By Lemma 8.5. $X^{\prime}(1 / k) \cong S^{3}$ for infinitely many $k$, and hence $X^{\prime}$ is a solid torus with meridian $0 / 1$. Since $\Delta(a / b, 0 / 1)=a>1$, this contradicts the fact that $X^{\prime}(a / b) \cong S^{3}$.

(2) $X$ is irreducible and not Seifert fibred. Since $\Delta(a / b, 0 / 1)=a>1$, the forms of $X(a / b)$ and $X(0 / 1)$ stated above contradict [CGLS] if $N \cong S^{3}$ and [BZ2, Corollary 1.4] otherwise.

(3) $X$ is Seifert fibred with incompressible boundary.

If $X$ is not the twisted $I$-bundle over the Klein bottle, let $\varphi$ be the slope on $\partial X$ of the Seifert fibre in the unique Seifert fibring of $X$. If $X$ is the twisted $I$-bundle over the Klein bottle, let $\varphi$ be the slope of the Seifert fibre in the Seifert structure on $X$ with orbifold $D^{2}(2,2)$. In both cases, $\varphi$ is the only slope on $\partial X$ such that $X(\varphi)$ is a non-trivial connected sum. Therefore, if $N \neq S^{3}$, then $\varphi=0 / 1$. But $X(a / b)$ is a lens space, and so $\Delta(a / b, 0 / 1)=1$, contradicting our assumption that $a>1$. Hence $N \cong S^{3}$, and so $\Delta(a / b, \varphi)=\Delta(0 / 1, \varphi)=1$. In particular $\varphi=1 / s$ for some integer $s$. Therefore $X(1 / s)$ is reducible. But this contradicts Lemma 8.5 .

\section{The Case $\Delta(\alpha, \beta)=6$ And the involution $\tau_{\alpha}$ REVERses the ORIENTATIONS OF THE SEIFERT FIBRES OF $M(\alpha)$}

In this section we suppose that Assumptions 5.1 hold and show that it is impossible for $\Delta(\alpha, \beta)$ to be 6 and for $\tau_{\alpha}$ to reverse the orientations of the Seifert fibres of $M(\alpha)$. We assume otherwise in order to obtain a contradiction. Here $M(\alpha) / \tau_{\alpha}=L(3, q) \cong L(3,1)$. By Lemma [5.9. $L$ is as shown in Figure 3. By Lemma 5.2 parts (2) and (3), $n$ is even, $m$ is odd, $|L|=1$, and $L_{\alpha}=L \cup K_{\alpha}$ is as shown in Figure 1. Since $L$ is a component of $L_{\alpha}$, we see that $L$ is a core of some 
Heegaard solid torus of $L(3,1)$. Hence the double branched cover of $(L(3,1), L)$ is homeomorphic to $L(3,1)$.

Let $Y, \mathcal{T}, X$ be as in the previous section, with $L(5,2 q)$ replaced by $L(3,1)$. Again as in that proof, here we have $X(a / b) \cong L(3,1)$ and $X(0 / 1) \cong L(3,1) \# N$ for some closed 3 -manifold $N$. In the current situation we only have the following weaker version of Lemma 8.5

Lemma 9.1. $X(1 / k)$ is irreducible for infinitely many $k \in \mathbb{Z}$.

Proof. As in the proof of Lemma 8.5, $\mathcal{T}(1 / k)$ is (the 3-braid $\sigma_{1}^{k} \sigma$ in $V$ ) $\cup V_{0}$, and $X(1 / k)=Q_{k} \cup \widetilde{V}_{0}$, where $Q_{k}$ is the double branched cover of $\left(V, \sigma_{1}^{k} \sigma\right)$ and $\widetilde{V}_{0}$ is a solid torus. Now $Q_{k}$ is a $T_{0}$-bundle over $S^{1}$, where $T_{0}$ is a once-punctured torus. If $\rho \in B_{3}$, let $\tilde{\rho}$ denote the corresponding homeomorphism $T_{0} \rightarrow T_{0}$. Then $\tilde{\sigma}_{1}$ and $\tilde{\sigma}_{2}$ are Dehn twists about a pair of curves in $T_{0}$ with intersection number 1 . With respect to this basis, $\tilde{\rho}$ defines an element of $S L_{2}(\mathbb{Z})$. Note that since $L$ is connected, $\sigma$ is not a power of $\sigma_{1}$. The elements of $S L_{2}(\mathbb{Z})$ corresponding to $\tilde{\sigma}_{1}^{k}$ and $\tilde{\sigma}$ are therefore $\left[\begin{array}{ll}1 & k \\ 0 & 1\end{array}\right]$ and $\left[\begin{array}{ll}a & b \\ c & d\end{array}\right]$, say, where $c \neq 0$. Then the matrix corresponding to $\tilde{\sigma}_{1}^{k} \tilde{\sigma}$ has trace $a+d+k c$, which has absolute value greater than 2 for all but at most five values of $k$. For such $k$ the manifold $Q_{k}$ is therefore hyperbolic.

Let $\beta$ be the boundary slope of the fibre of $Q_{k}$; note that $\beta$ projects to the meridian $\mu$ of $V$. Let $\mu_{0}, \tilde{\mu}_{0}$ be the meridians of $V_{0}, \widetilde{V}_{0}$, respectively. Since $\Delta\left(\mu, \mu_{0}\right)=3$, we have $\Delta\left(\beta, \tilde{\mu}_{0}\right)=3$. If $Q_{k}$ is hyperbolic, then by [BZ1, Lemma 4.1] $Q_{k}(\gamma)$ reducible implies $\Delta(\beta, \gamma)=1$. Therefore $X(1 / k)=Q_{k}\left(\tilde{\mu}_{0}\right)$ is irreducible for infinitely many $k$.

As in the previous section, we have possibilities (1), (2) and (3) for $X$. Cases (1) and (2) are ruled out exactly as before (applying Lemma 9.1 instead of Lemma 8.5). In case (3) we may conclude that both $X(a / b)$ and $X(0 / 1)$ are $L(3,1), X(1 / s)$ is reducible for some integer $s$ and $\Delta\left(\beta, \tilde{\mu}_{0}\right)=3$. The proof of Lemma 8.3 shows that the monodromy of the once-punctured torus bundle $Q_{s}$ has order 3. Therefore $Q_{s}$ has base orbifold $D^{2}(3,3)$, and so $X(1 / s) \cong Q_{s}\left(\tilde{\mu}_{0}\right) \cong L\left(3, q_{1}\right) \# L\left(3, q_{2}\right)$. This implies that $X$ has base orbifold $D^{2}(3,3)$. But then no two distinct fillings on $X$ can give the lens space $L(3,1)$, yielding a contradiction.

\section{The CASe $\Delta(\alpha, \beta)=5$ And the involution $\tau_{\alpha}$ REVERSES The ORIENTATIONS OF THE SEIFERT FIBRES OF $M(\alpha)$}

In this section we suppose that Assumptions 5.1 hold and show that it is impossible for $\Delta(\alpha, \beta)$ to be 5 and for $\tau_{\alpha}$ to reverse the orientations of the Seifert fibres of $M(\alpha)$. We assume otherwise in order to obtain a contradiction.

As in 97 we just need to show that the two knots, $K, K^{\prime}$, shown in Figures 26 and 27, respectively, are inequivalent in $S^{3}$.

Theorem 10.1. The knots $K$ and $K^{\prime}$ are inequivalent.

As in 97 , we will show that the double branched covers $W, W^{\prime}$ of $\left(S^{3}, K\right),\left(S^{3}, K^{\prime}\right)$ are not homeomorphic.

Here we consider the tangle $\mathcal{T}=(R, t)$ shown in Figure 28, with double branched cover $X$. Let the boundary components of $R$ be $S, S_{1}, S_{2}$ (see Figure 28) and the corresponding boundary components of $X$ be $G, T_{1}, T_{2}$, so that $T_{1}$ and $T_{2}$ are tori and $G$ has genus two. 


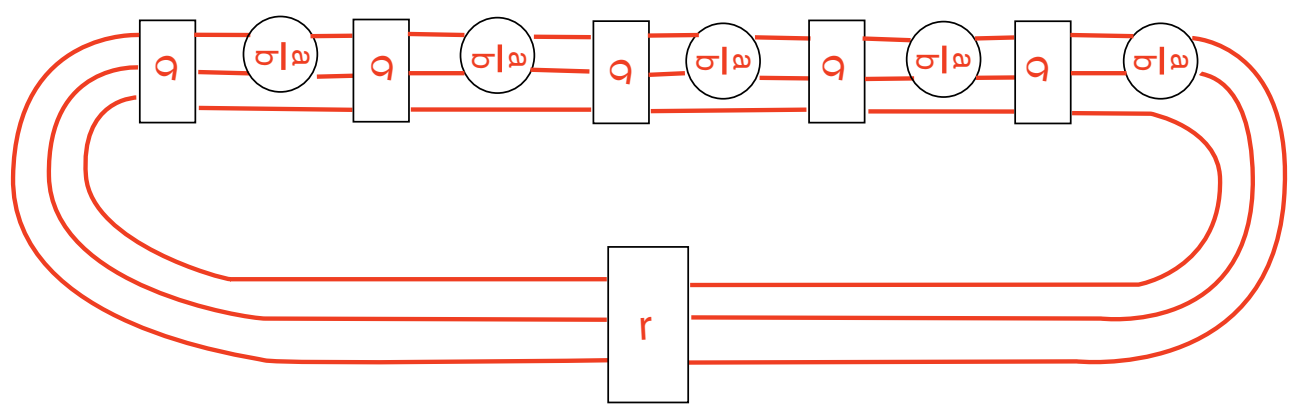

FiguRE 26

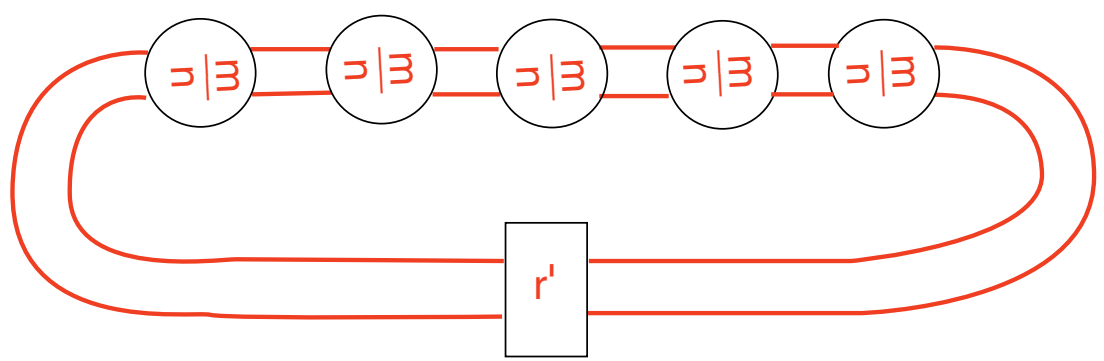

FiguRE 27

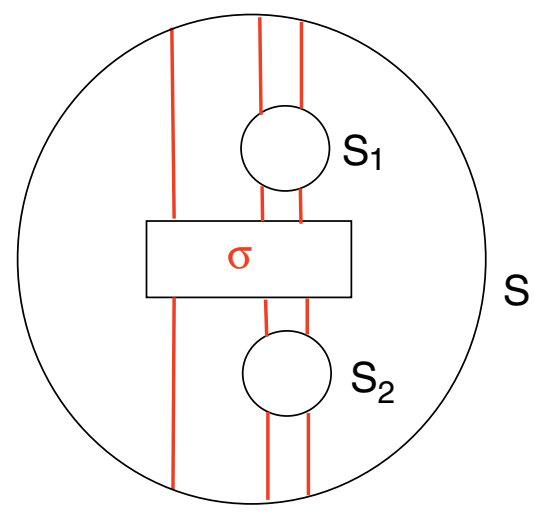

FiguRE 28

Lemma 10.2. If $G$ is compressible in $X$ then $\mathcal{T}$ is isotopic to the tangle shown in Figure 29.

Proof. Since $t \cup S_{1} \cup S_{2}$ is connected, any essential disk $D$ in $\mathcal{T}$ with $\partial D \subset S$ must meet $t$ in a single point. Hence $D$ meets the unique strand of $t$ connecting $S_{1}$ and $S_{2}$, decomposing $\mathcal{T}$ into two tangles $\mathcal{T}_{1}$ and $\mathcal{T}_{2}$. We claim that each of $\mathcal{T}_{1}$ and $\mathcal{T}_{2}$ is a product tangle. To see this, note that deleting the strand of $t$ that joins $S_{2}$ to $S$ and runs through the braid $\sigma$ gives the tangle shown in Figure 30. It follows that $\mathcal{T}_{1}$ is as stated. Similarly, $\mathcal{T}_{2}$ is also a product tangle. 


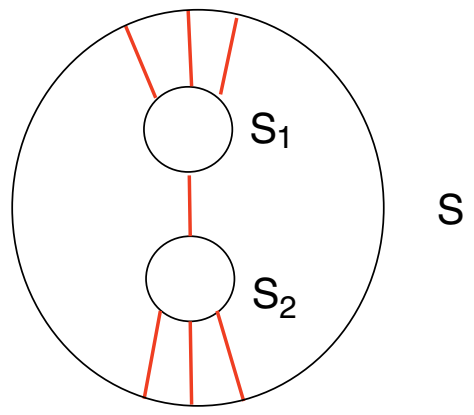

FiguRE 29

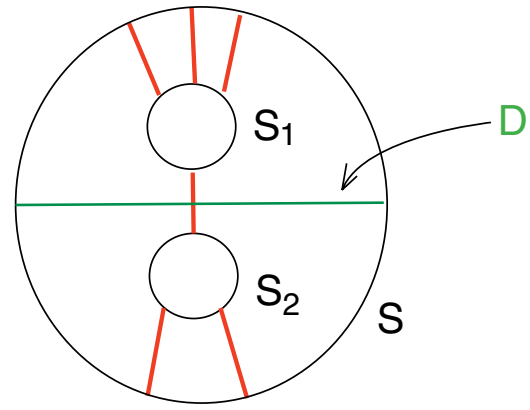

FIGURE 30

Corollary 10.3. If $G$ is compressible in $X$ then $X(a / b, a / b)$ is a genus 2 handlebody.

Lemma 10.4. If $G$ is incompressible in $X$ then $G$ is incompressible in $X(a / b, a / b)$.

Proof. This is exactly like the proof of Lemma 7.6 in 97 using the annuli $A_{1}$ and $A_{2}$ shown in Figure 31.

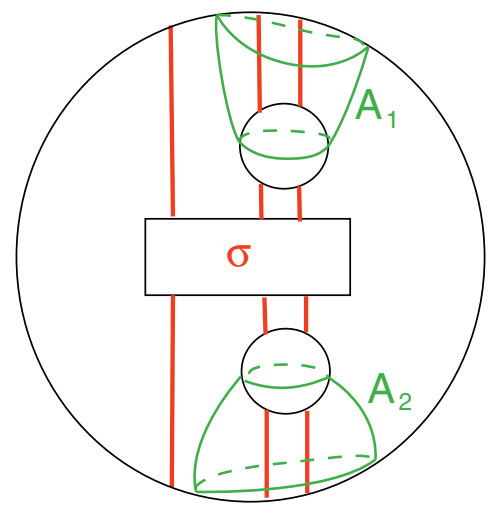

FIGURE 31 
Proposition 10.5. $W$ either

(1) contains a separating incompressible surface of genus 2 , or

(2) has Heegaard genus at most 3.

Proof. From Figure 32 we see that $W \cong U \cup_{G} Z(a / b) \cup_{G^{\prime}} U^{\prime}$, where $U$ and $U^{\prime}$ are copies of $X(a / b, a / b)$.

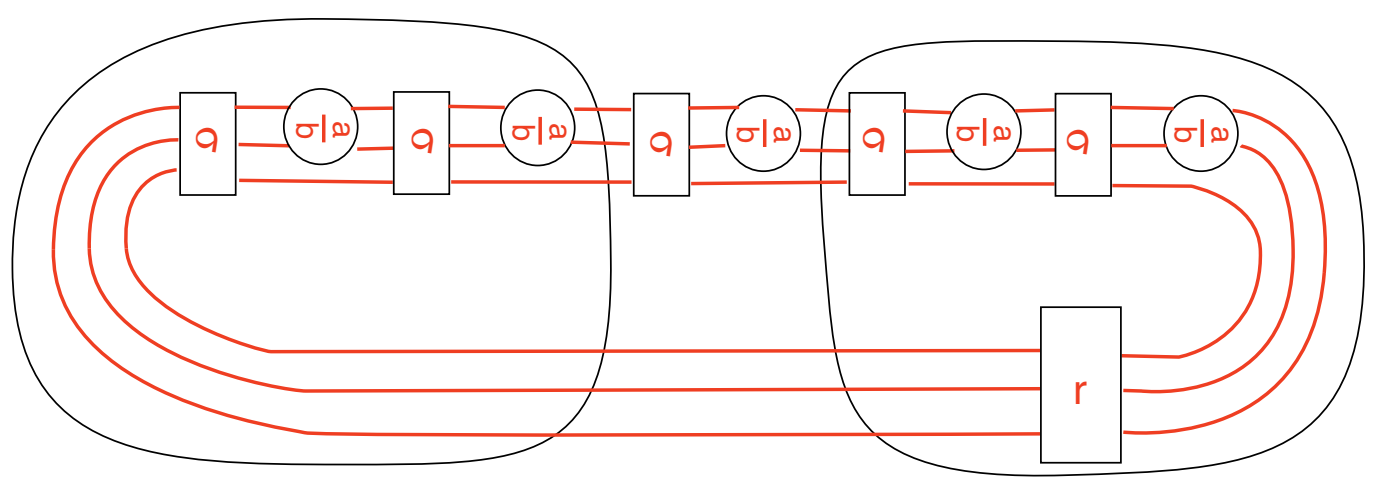

FiguRE 32

If $G$ is incompressible in $X$, then we get conclusion (1) by Lemmas 10.4 and 7.7 .

If $G$ is compressible in $X$, then we get conclusion (2) by Corollary 10.3 and the proof of part (3) of Proposition 7.8 .

Proof of Theorem 10.1. Assume $W \cong W^{\prime}$. Since $W^{\prime}$ is a Seifert fibre space over $S^{2}$ with 5 exceptional fibres, we get a contradiction to Proposition 10.5 as in the proof of Theorem 7.1 in cases (1) and (3) of Proposition 7.8.

\section{A family of examples Realizing $\Delta(\alpha, \beta)=4$}

In this section we show that distance 4 between a prism manifold filling slope and a once-punctured torus slope can be realized on infinitely many hyperbolic knot manifolds.

Let $W h$ be the exterior of the Whitehead link with standard meridian-longitude coordinates on $\partial W h$. We use $W h(\gamma)$ to denote the manifold of Dehn filling one boundary component of $W h$ with slope $\gamma$, and $W h(\gamma, \delta)$ the manifold of Dehn filling one boundary component with slope $\gamma$ and the other with slope $\delta$.

Theorem 11.1. For each integer $n$ with $|n|>1, W h\left(\frac{-2 n \pm 1}{n}\right)$ is a hyperbolic knot manifold whose 0-slope is the boundary slope of an essential once-punctured torus and whose -4-slope yields a prism manifold whose base orbifold is $S^{2}(2,2, \mid \mp 2 n-$ $1 \mid)$.

Proof. It is well known that $W h(\gamma)$ is hyperbolic for each $\gamma \notin\{-1,-2,-3,-4,0$, $1 / 0\}$. That $W h(\gamma), \gamma \neq 1 / 0$, contains an essential once-punctured torus with boundary slope 0 is obvious from the Whitehead link diagram.

The Whitehead link admits an involution $\tau$ as shown in Figure 33 . This involution restricts to an involution, still denoted $\tau$, on $W h$ and then extends to an involution $\tau_{\gamma}$ on $W h(\gamma)$ and to an involution $\tau_{\gamma, \delta}$ on $W h(\gamma, \delta)$ for all slopes $\gamma$ and $\delta$. The quotient space under $\tau$ is shown in Figure 34. Note that the branch 


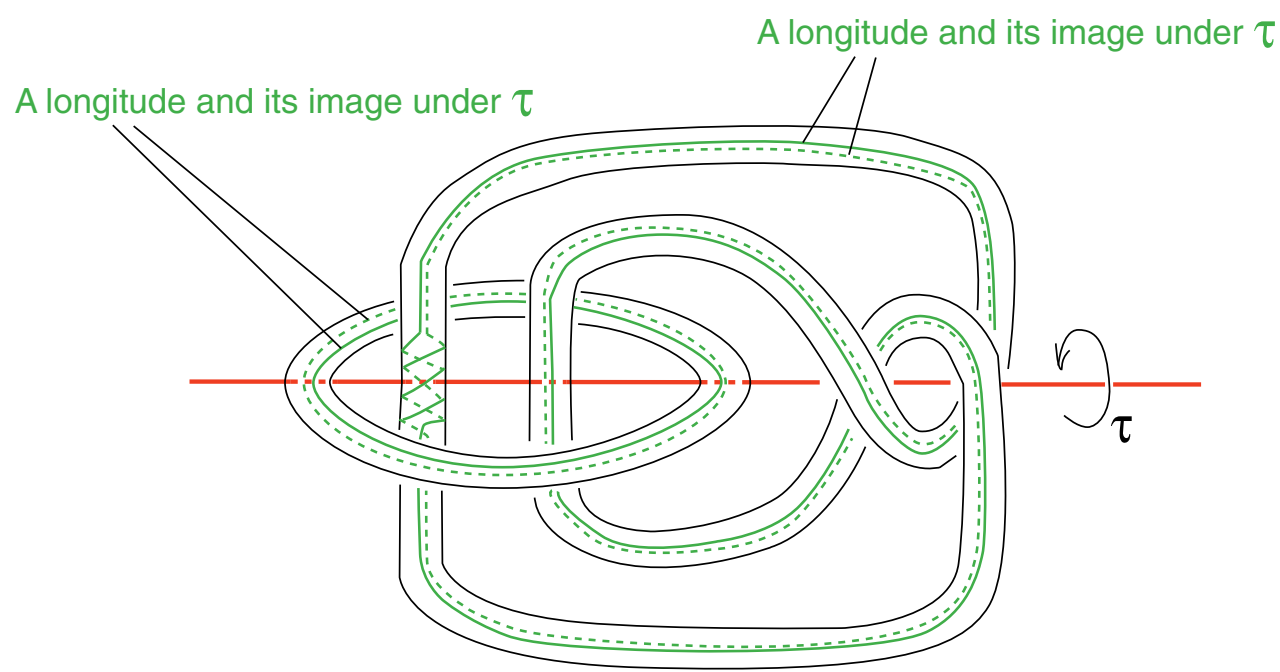

FIGURE 33

set of $W h(\gamma) / \tau_{\gamma}$ is obtained by removing the two 1/0-tangles in Figure 34 and then filling one $\gamma$-tangle. Figure 35 shows the branch set in $W h(-4) / \tau_{-4}$ and Figure 36 shows the branch set in $W h\left(\frac{-2 n \pm 1}{n},-4\right) / \tau_{\frac{-2 n \pm 1}{n},-4}$. As the branch set in $W h\left(\frac{-2 n \pm 1}{n},-4\right) / \tau_{\frac{-2 n \pm 1}{n},-4}=S^{3}$ is a Montesinos link of type $\left(2,2, \frac{\mp 2 n-1}{2}\right)$, the double branched cover $W h\left(\frac{-2 n \pm 1}{n},-4\right)$ is a prism manifold whose base orbifold is $S^{2}(2,2,|\mp 2 n-1|)$.

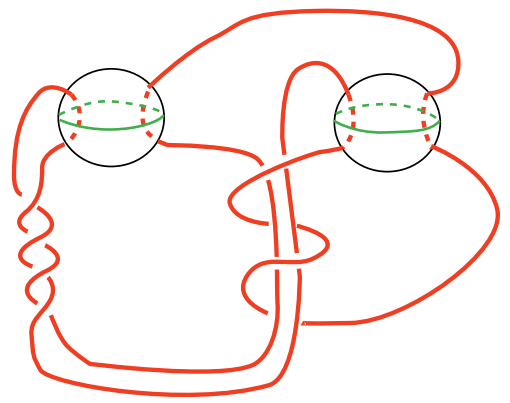

FigURE 34

12. The CASE When $\Delta(\alpha, \beta)=4$ AND $M(\alpha)$ IS A PRISM MANIFOLD

In this section we show

Theorem 12.1. Let $M$ be a hyperbolic knot exterior containing an essential oncepunctured torus with slope $\beta$. If $M(\alpha)$ is a prism manifold with $\Delta(\alpha, \beta)=4$, then $M$ is one of the examples given in $₫ 11$, that is, $(M ; \alpha, \beta) \cong\left(W h\left(\frac{-2 n \pm 1}{n}\right) ;-4,0\right)$ for some integer $n$ with $|n|>1$. 

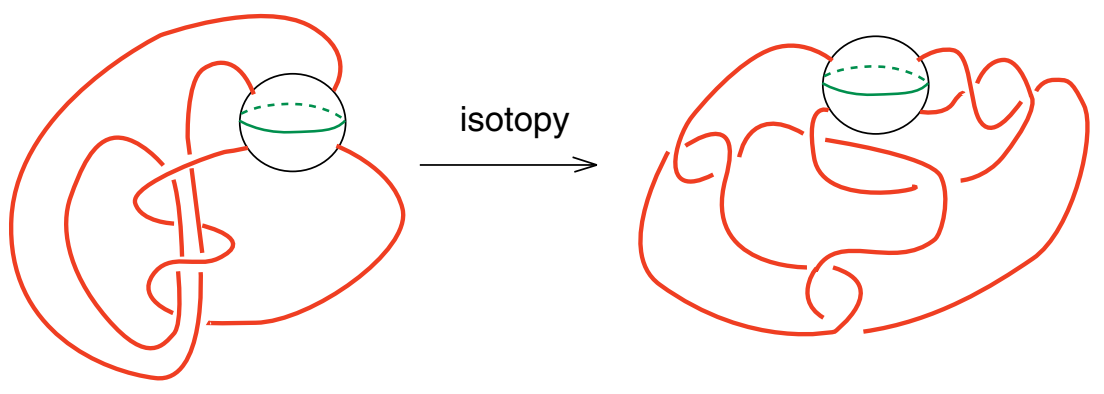

FiguRE 35

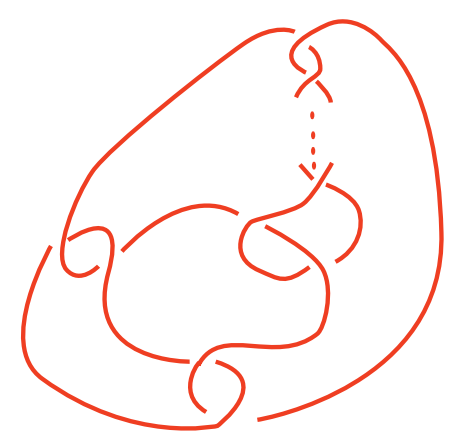

FIGURE 36

Let $F$ be an essential once-punctured torus in $M$ with slope $\beta$. Choose a Klein bottle $\hat{P}$ in $M(\alpha)$ which has the minimal number of intersection components with $\partial M$ and let $P=M \cap \hat{P}$. Then $p=|\partial P|>0$ since $M$ is hyperbolic. The punctured Klein bottle $P$ is essential in $M$, i.e. it is incompressible and boundaryincompressible in $M$. The proof of this statement is essentially contained in [Te2, Proofs of Lemmas 2.1 and 2.2], and we only need to add the condition that $M(\alpha)$ is a prism manifold which is thus irreducible and does not contain a projective plane.

As usual, the two surfaces $F$ and $P$ define two labeled intersection graphs which we denote by $\Gamma_{F}$ and $\Gamma_{P}$. Then neither $\Gamma_{F}$ nor $\Gamma_{P}$ contain trivial loops ([Te2, Lemma 3.1] with the same proof). The graph $\Gamma_{F}$ has a unique vertex whose valency is $4 p$, and the graph $\Gamma_{P}$ has $p$ vertices each having valency 4 . Note that every edge of $\Gamma_{F}$ is positive since $F$ is orientable and has only one boundary component.

Lemma 12.2. (1) When $p \geq 2, \Gamma_{F}$ has no $S$-cycle.

(2) When $p \geq 3, \Gamma_{F}$ has no generalized $S$-cycle (see [Te2] for its definition).

(3) $\Gamma_{F}$ cannot have more than $\frac{p}{2}+1$ mutually parallel edges.

Proof. Part (1) is [Te2, Lemma 3.2] with the same proof, part (2) is [Te2, Lemma 3.3] with a similar argument plus the fact that $M(\alpha)$ does not contain a projective plane, and part (3) is [LT, Lemma $6.2(4)]$ with the same proof.

Lemma 12.3. $p=1$.

Proof. The lemma was proved in [Te2, Lemma 5.2] when $M$ was a genus one noncabled knot exterior in $S^{3}$, in which case $p$ was an odd integer. In our situation, 
we need to extend the argument of [Te2, Lemma 5.2] slightly, using Lemma 12.2 (3) instead of [Te2, Lemma 3.4].

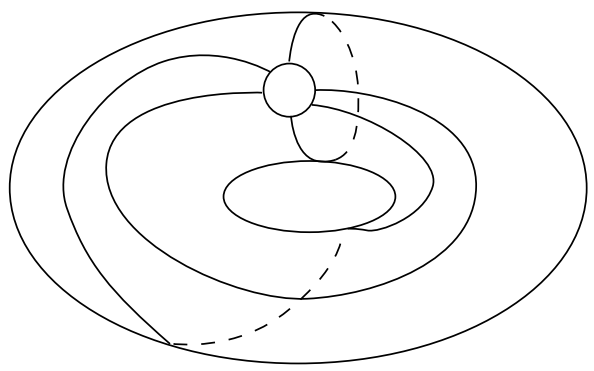

FIGURE 37

Suppose otherwise that $p \geq 2$. The reduced graph $\bar{\Gamma}_{F}$ is a subgraph of the graph shown in Figure 37 (Go1, Lemma 5.1]). In particular $\bar{\Gamma}_{F}$ has at most three edges. Suppose these edges of $\bar{\Gamma}_{F}$ have weights $w_{k}, k=1,2,3$, some of which may possibly be zero. Then $2\left(w_{1}+w_{2}+w_{3}\right)=4 p$. Let $e_{1}, \ldots, e_{w_{k}}$ be a parallel family of consecutive edges in $\Gamma_{F}$. Reading the labels around the vertex of $\Gamma_{F}$, we see that the labels of the edges $e_{1}, e_{2}, \ldots, e_{w_{k}}$ are as illustrated in Figure 38 .

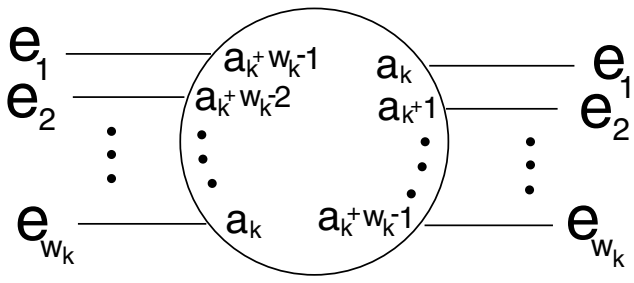

FIGURE 38

By Lemma 12.2, $w_{k}=0$ or $1,1 \leq k \leq 3$. (More precisely, this follows from Lemma 12.2 (1) if $w_{k}$ is even, Lemma 12.2 (2) if $w_{k}$ is odd and $p \geq 3$, and Lemma 12.2 (3) if $w_{k}$ is odd and $p=2$.) This is a contradiction.

So $\Gamma_{F}$ has exactly two edges and both are level edges (i.e. having the same label at the two endpoints of the edge). Let $e_{1}, e_{2}$ be the two edges of $\Gamma_{F}$ and of $\Gamma_{P}$. Note that each $e_{i}$ is an orientation-reversing loop in $P$ by the parity rule.

Since $\Delta(\alpha, \beta)=4$, if the endpoints of the two edges around the vertex $\partial F$ are labeled consecutively by $1,2,3,4$, the labels around $\partial P$ are also consecutive. It follows from this fact that if the two edges in $\Gamma_{F}$ are not parallel, then the two edges in $\Gamma_{P}$ must be parallel. Also, combining this fact with the proof of $[\mathrm{Te} 2$, Lemma 4.1], we have that the two edges $e_{1}$ and $e_{2}$ cannot be parallel in both $\Gamma_{P}$ 


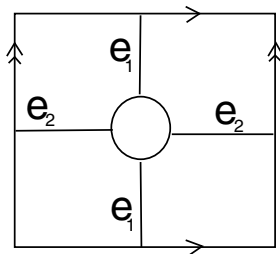

$\Gamma_{\mathrm{F}}$

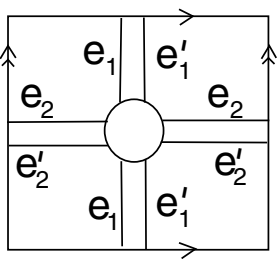

$\Gamma_{\mathrm{F}}^{\prime}$

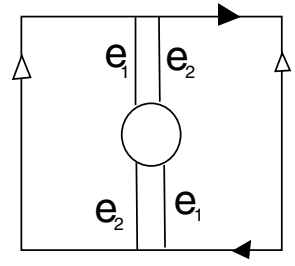

$\Gamma_{\mathrm{P}}$

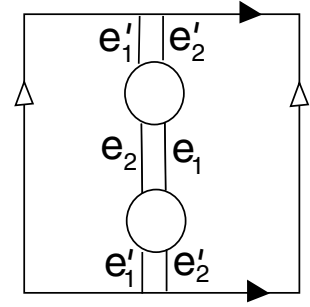

$\Gamma_{\mathrm{S}}$

FigURE 39

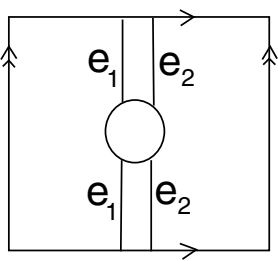

$\Gamma_{\mathrm{F}}$

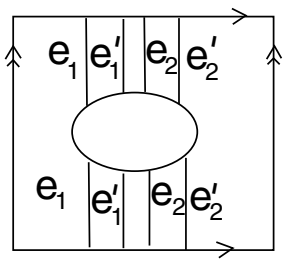

$\Gamma_{\mathrm{F}}^{\prime}$

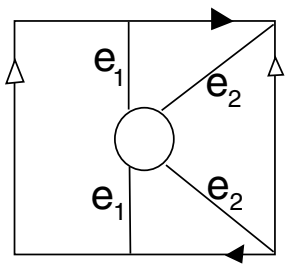

$\Gamma_{\mathrm{P}}$

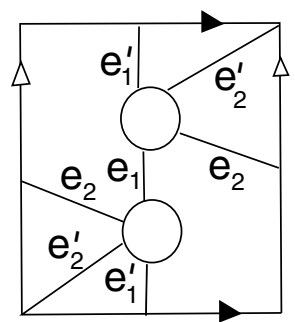

$\Gamma_{\mathrm{S}}$

FIGURE 40

and $\Gamma_{F}$. So there are only two possible configurations for the pair of graphs $\Gamma_{F}$ and $\Gamma_{P}$, which we illustrate in Figure 39 and Figure 40, respectively. 
Let $S$ be the frontier of a thin regular neighbourhood of $P$ in $M$. Then $S$ is a separating twice-punctured torus in $M$. The surfaces $F$ and $S$ define two labeled intersection graphs $\Gamma_{F}^{\prime}$ and $\Gamma_{S}$. Note that $\Gamma_{F}^{\prime}$ is obtained by doubling the edges of $\Gamma_{F}$ and $\Gamma_{S}$ double covers $\Gamma_{P}$. See Figures 39 and 40 for illustrations of the graphs $\Gamma_{F}, \Gamma_{P}, \Gamma_{F}^{\prime}$ and $\Gamma_{S}$.

The surface $S$ separates $M$ into two components which we denote by $X^{+}$and $X^{-}$, where $X^{-}$is a twisted $I$-bundle over $P$. Note that $\widehat{X}^{-}$is a twisted $I$-bundle over the Klein bottle $\widehat{P}$ and $\widehat{X}^{+}$is a solid torus since $M(\alpha)=\widehat{X}^{-} \cup \widehat{X}^{+}$is a prism manifold. Let $H^{\epsilon}$ denote the part of the filling solid torus of $M(\alpha)$ contained in $\widehat{X}^{\epsilon}, \epsilon \in\{ \pm\}$, and let $\partial_{0} H^{\epsilon}=\partial H^{\epsilon} \cap \partial M$.

We first show

Lemma 12.4. The case given by Figure 39 cannot occur.

Proof. The 4-gon face of $\Gamma_{F}^{\prime}$, which we denote by $f$, is contained in $X^{+}$and its boundary edges form a Scharlemann cycle of order 4 . From Figure 39 we see that $\partial f$ is a non-separating curve in the genus two surface $S \cup \partial_{0} H^{+}$and $\partial f \cap S$ is contained in an essential annulus $A$ in $\widehat{S}$. Let $U$ be a regular neighbourhood of $A \cup H^{+} \cup f$ in $\widehat{X}^{+}$. Then $U$ is a compact 3-manifold with $\partial U$ a torus, and the fundamental group of $U$ has the presentation

$$
\left\langle x, t: x^{3} t x t=1\right\rangle,
$$

where we take a fat base point in $\widehat{S}$ containing $\partial S \cup e_{1} \cup e_{2}, x$ is a based loop formed by a cocore arc of $\partial_{0} H^{+}$and $t$ is represented by a core circle of $A$. Let $y=x t$; then

$$
\pi_{1}(U)=\left\langle x, y: x^{2} y^{2}=1\right\rangle .
$$

So $U$ is Seifert fibred with base orbifold $D(2,2)$. Thus $U$ contains a Klein bottle. But $U$ is contained in the solid torus $\widehat{X}^{+}$. This gives a contradiction.

So the case of Figure 40 must occur. In this case we are going to show that $M$ is obtained by Dehn filling one boundary component of the Whitehead link exterior.

In this case, the bigon faces of $\Gamma_{F}^{\prime}$ between $e_{1}$ and $e_{1}^{\prime}$ and between $e_{2}$ and $e_{2}^{\prime}$ lie in $X^{-}$, and the bigon face between $e_{1}^{\prime}$ and $e_{2}$, which we denote by $B$, is contained in $X^{+}$. Let $Q$ be a regular neighbourhood of $S \cup \partial_{0} H^{+} \cup B$ in $X^{+}$, and $\widehat{Q}=Q \cup H^{+}$. Then it's easy to see that $\widehat{Q}$ is a Seifert fibred manifold whose base orbifold is an annulus with a single cone point of order 2 . The boundary of $\widehat{Q}$ consists of two tori, one of which is the torus $\widehat{S}$. Let $T_{0}$ be the other component. Note that $T_{0}$ is contained in the interior of $X^{+}$. Since $\widehat{X}^{+}$is a solid torus, $T_{0}$ must bound a solid torus in $\widehat{X}^{+} \backslash \widehat{Q}$, which we denote by $N$.

Lemma 12.5. The Seifert structure of $\widehat{Q}$ does not match with the Seifert structure of $\widehat{X}^{-}$whose base orbifold is $D(2,2)$.

Proof. The $S$-cycle $\left\{e_{1}, e_{1}^{\prime}\right\}$ in $\Gamma_{F}^{\prime}$ implies that as a cycle in $\Gamma_{S}, e_{1} \cup e_{1}^{\prime}$ is a fibre of the Seifert structure of $\widehat{X}^{-}$whose base orbifold is $D(2,2)$. Similarly the $S$-cycle $\left\{e_{1}^{\prime}, e_{2}\right\}$ in $\Gamma_{F}^{\prime}$ implies that as a cycle in $\Gamma_{S}, e_{1}^{\prime} \cup e_{2}$ is a fibre of the Seifert structure of $\widehat{Q}$. Obviously from Figure 40 these two cycles have different slopes in $\widehat{S}$.

Let $W=X^{-} \cup_{S} Q$. Note that $M=W \cup_{T_{0}} N$. So we just need to show that $W$ is the Whitehead link exterior. We use the notation $W(\partial M, \gamma)$ to denote the Dehn filling of $W$ along a slope $\gamma$ in $\partial M \subset \partial W$. 
Lemma 12.6. (1) $W$ is irreducible.

(2) The twice-punctured torus $S$ is incompressible in $W$.

(3) $F \cap W$ has a component which is an essential once-punctured annulus in $W$ with the puncture lying in $\partial M$ of slope $\beta$ and with the boundary of the annulus lying in $T_{0}$.

(4) $W(\partial M, \alpha)$ contains an essential torus which is $\widehat{S}$.

Proof. By the construction of $Q$, one can easily see that $Q$ is irreducible and $S$ is incompressible in $Q$. Obviously $X^{-}$is irreducible and $S$ is incompressible in $X^{-}$. Thus $S$ is incompressible in $W=X^{-} \cup_{S} Q$ and $W$ is irreducible. So we get (1) and (2).

Part (3) follows from the graph $\Gamma_{F}^{\prime}$ shown in Figure 40 and the construction of $Q$. In fact, the exterior in $F$ of the annulus which is the annulus face of $\Gamma_{F}^{\prime}$ shrunk slightly into the interior of the face is the required punctured annulus. It is incompressible in $W$ because it is an essential subsurface of $F$. It is boundary incompressible in $W$ because it has only one intersection component with $\partial M$ and $M$ does not contain an essential disk with slope $\beta$.

For (4), we just need to note that $W(\partial M, \alpha)=\widehat{X}^{-} \cup_{\widehat{S}} \widehat{Q}$.

Lemma 12.7. $W$ is hyperbolic.

Proof. We already know that $W$ is irreducible (Lemma 12.6(1)). Obviously $W$ cannot be Seifert fibred since $M=W \cup N$ is hyperbolic. So we just need to show that $W$ is atoroidal. Suppose otherwise that $W$ contains an essential torus $T$. Note that $T$ is separating since $M$ is hyperbolic.

Note that $Q$ (a compression body) is of the form $T_{0} \times[0,1]$ union a 1-handle attached to $T_{0} \times 1$. It is now easy to see that any incompressible torus in $Q$ is isotopic into $T_{0} \times[0,1]$, and therefore boundary parallel. Hence $T$ cannot be contained in $Q$. Obviously $X^{-}$is atoroidal because it is a twisted $I$-bundle over a punctured Klein bottle. So $T$ cannot be contained in $X^{-}$either. Therefore $T$ must intersect $S$. As $S$ is incompressible in $W$ (Lemma 12.6(2)), we may assume that every component of $S \cap T$ is a circle which is essential in both $T$ and $S$. As $S$ is separating, $T \cap S$ has an even number of components. We may further assume that each component of $T \backslash(S \cap T)$ is an essential annulus in $\left(X^{-}, S\right)$ or in $(Q, S)$ (using isotopy of $T$ to eliminate inessential ones), and thus can be further assumed to be a vertical annulus in the characteristic $I$-bundle of $\left(X^{-}, S\right)$ or $(Q, S)$. Note that the characteristic $I$ bundle for the pair $(Q, S)$ is isotopic to a regular neighbourhood of $B \cup \partial_{0} H^{+}$in $Q$ such that the horizontal boundary of the $I$-bundle is a twice-punctured annulus $\phi$ contained in $S$ such that $\widehat{\phi}$ is an essential annulus in $\widehat{S}$, and the vertical boundary of the $I$-bundle has two components: one is $\partial_{0} H^{+}$and the other is the frontier of the $I$-bundle in $Q$. So we may assume that $S \cap T$ is contained in $\phi$.

Let $A$ be a component of $T \backslash(T \cap S)$. It's easy to see that $\partial A$ is $\widehat{S}$-essential, for otherwise $A$ would be isotopic to $\partial_{0} H^{\epsilon}$ and $T$ would be parallel to $\partial M$. Now if $A$ is contained in $Q$, its two boundary components are either isotopic in $\phi$ to the two inner boundary components of $\phi$ respectively or bound an annulus in $\phi$ which separates $\phi$ into two once-punctured annuli. Moreover, $A$ is a vertical annulus in the Seifert fibred structure of $\widehat{Q}$. If $A$ is contained in $X^{-}$, it is a vertical annulus in one of the two Seifert fibred structures of $\widehat{X}^{-}$. So the Seifert structure of $\widehat{Q}$ matches a Seifert structure of $\widehat{X}^{-}$. By Lemma 12.5 the Seifert structure of $\widehat{X}^{-}$ must be the one whose base orbifold is a Möbius band. Thus if a component $A$ of 


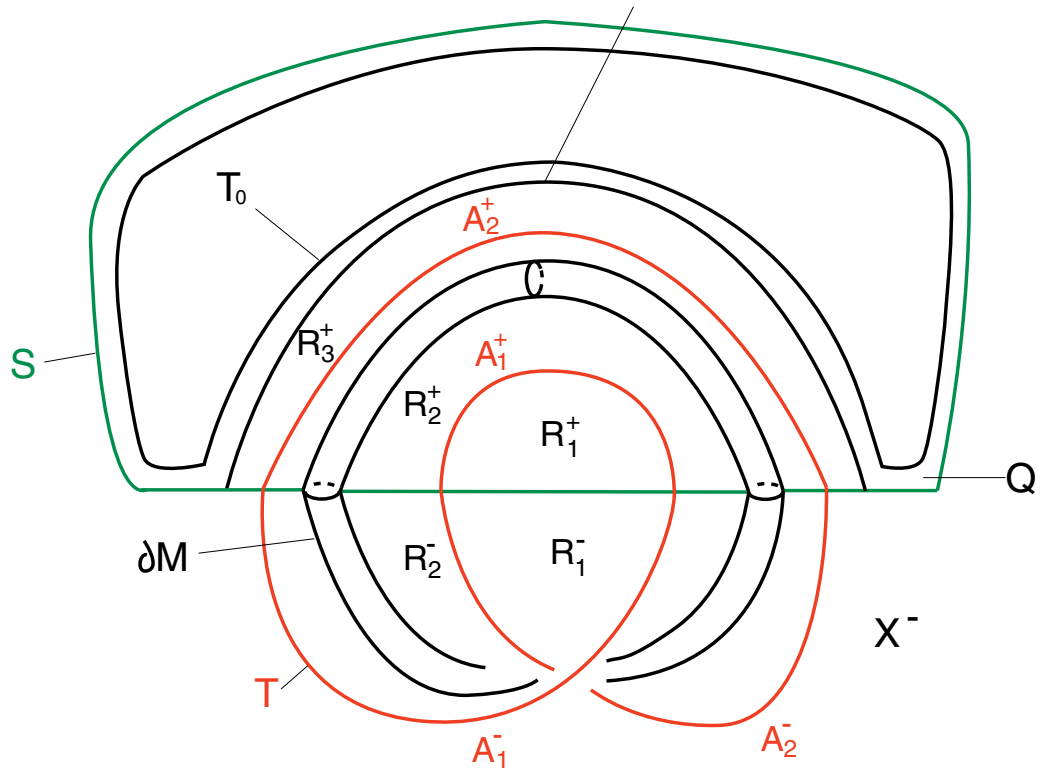

FIGURE 41

$T \backslash(S \cap T)$ is contained in $X^{-}$, it is a non-separating annulus in $X^{-}$. In particular, if $A$ is contained in $X^{-}, \partial A$ cannot be parallel in $S$. For otherwise the union of $A$ with the annulus in $S$ bounded by $\partial A$ would be a Klein bottle in $W \subset M$, giving a contradiction.

With the above information we have obtained on the components of $T \backslash(S \cap T)$ we see that the following case must occur: $T \backslash(S \cap T)$ has exactly four components, two in $Q$ which we denote by $A_{1}^{+}$and $A_{2}^{+}$, and two in $X^{-}$which we denote by $A_{1}^{-}$and $A_{2}^{-}$, and they are connected as shown in Figure 41. More specifically, the annuli $A_{1}^{+}$and $A_{2}^{+}$separate $Q$ into three components $R_{1}^{+}, R_{2}^{+}, R_{3}^{+}$such that $R_{1}^{+}$is a solid torus in which $A_{1}^{+}$has winding number $2, R_{2}^{+}$contains $\partial_{0} H^{+}$and is a product $I$-bundle over a once-punctured annulus, and $R_{3}$ is a regular neighbourhood of $T_{0}$. The annuli $A_{1}^{-}$and $A_{2}^{-}$separate $X^{-}$into two components $R_{1}^{-}, R_{2}^{-}$such that $R_{1}^{-}$ contains $\partial_{0} H^{-}$and is a product $I$-bundle over a once-punctured annulus, and $R_{2}^{-}$ is a solid torus (cf. Figure 41). Moreover, $R_{2}^{+} \cup R_{2}^{-}$is a once-punctured annulus bundle over $S^{1}$ with finite order monodromy and thus is Seifert fibred. In fact, one can see that the monodromy has order two. On the other hand, $R_{1}^{+} \cup R_{1}^{-} \cup R_{3}^{+}$ is Seifert fibred over an annulus with one cone point of order two. Hence $W$ is a graph manifold. But $M=W \cup N$ is hyperbolic. We get a contradiction.

Lemma 12.8. $W(\partial M, \beta)$ contains an essential annulus which is the cap-off of the once-punctured annulus given in part (3) of Lemma 12.6.

Proof. Note that the punctured annulus given in part (3) of Lemma 12.6 is nonseparating in $W$. So it caps off to a non-separating annulus in $W(\partial M, \beta)$. If this annulus is inessential in $W(\partial M, \beta)$, then it must be compressible, from which we 
may get a compressing disk for $T_{0}$ in $W(\partial M, \beta)$. That is, $\beta$ becomes a boundaryreducing Dehn filling slope on $\partial M$ for $W$. On the other hand, $\alpha$ is a toroidal filling slope on $\partial M$ for $W$ by Lemma 12.6(4). Hence by GL, we have $\Delta(\alpha, \beta) \leq 2$. But this contradicts the assumption that $\Delta(\alpha, \beta)=4$. Thus the above annulus is essential in $W(\partial M, \beta)$.

Now we have shown that $W$ is hyperbolic, and for $(W, \partial M), \alpha$ is a toroidal filling slope and $\beta$ an annular filling slope. Furthermore $W(\partial M, \beta)$ contains an essential annulus whose intersection with $\partial M$ has only one component. Applying GW2, Theorem 1.1], we see that $W$ is the Whitehead link exterior.

So $W \cong W h$. By tubing off the once-punctured annulus in $W$ (given by Lemma 12.6 (3)) with an annulus in $T_{0}$, we get a once-punctured torus in $(W, \partial M)$ with slope $\beta$. So $\beta$ corresponds to the zero slope with respect to the standard coordinates on $\partial W h$. Similarly we see that $\alpha$ is the slope -4 . As $\widehat{Q}$ is Seifert fibred over an annulus with a single cone point, $\widehat{X}^{+}=\widehat{Q} \cup \cup_{T_{0}} N$ is a solid torus if and only if the filling slope on $T_{0}$ is distance one from the Seifert slope of $\widehat{Q}$ on $T_{0}$. This Seifert slope is unique. From the examples given in $\$ 11$, we see that the Seifert slope of $\widehat{Q}$ on $T_{0}$ is -2 and those examples are the only examples realizing Theorem 1.3(1). That is, we have $(M ; \alpha, \beta) \cong\left(W h\left(\frac{-2 n \pm 1}{n}\right) ;-4,0\right)$ for some integer $n$ with $|n|>1$.

\section{Proof of Theorems 1.4 and 1.5}

Proof of Theorem 1.4. Let $M$ be a hyperbolic knot manifold containing an essential once-punctured torus $F_{\beta}$ with boundary slope $\beta$. Let $\gamma$ be an exceptional slope on $\partial M$.

We may suppose that the capped-off torus $\hat{F}_{\beta}$ is incompressible in $M(\beta)$ by Proposition 3.1. Now $M(\gamma)$ is either reducible, small Seifert, or toroidal. In the first case $\Delta(\beta, \gamma)=1$ by [BZ1, Lemma 4.1], while in the second case Theorem 1.3 implies that $\Delta(\beta, \gamma) \leq 5$ with equality only if $(M ; \gamma, \beta) \cong(W h(-3 / 2) ;-5,0)$ and $M(\gamma)$ has base orbifold $S^{2}(2,3,3)$, and $\Delta(\beta, \gamma)=4$ only if $(M ; \gamma, \beta) \cong\left(W h\left(\frac{-2 n \pm 1}{n}\right) ;-4,0\right)$ for some integer $n$ with $|n|>1$ and $M(\gamma)$ has base orbifold $S^{2}(2,2,|\mp 2 n-1|)$.

So suppose that $M(\gamma)$ is toroidal. We then have a punctured torus $F_{\gamma}$ in $M$ with boundary slope $\gamma$ such that the capped-off torus $\hat{F}_{\gamma}$ in $M(\gamma)$ is incompressible. Assume that $n_{\gamma}$, the number of boundary components of $F_{\gamma}$, is minimal over all such punctured tori. Similarly, assuming for the moment only that $M(\beta)$ is toroidal, we have a punctured torus $F_{\beta}$ in $M$ with boundary slope $\beta$ and $n_{\beta}$ boundary components. Triples $\left(M ; F_{\beta}, F_{\gamma}\right)$ of this kind with $\Delta(\beta, \gamma) \geq 4$ are classified in Go1] (in the case $\Delta(\beta, \gamma) \geq 6$ ) and [GW] (in the case $\Delta(\beta, \gamma)=4$ or 5 ). In particular, it is shown in $\mathrm{GW}$ ] that if $M$ is a hyperbolic knot manifold with a once-punctured torus slope $\beta$ and a toroidal slope $\gamma$ with $\Delta(\beta, \gamma)=4$, then $(M ; \gamma, \beta) \cong(W h(\delta) ;-4,0)$ for some slope $\delta$ on the other boundary component of $W h$. This proves part (3)(a) of the theorem.

The only examples with $n_{\beta}=1$ and $\Delta(\beta, \gamma) \geq 5$ are $M=W h(-5 / 2)$, with $\Delta(\beta, \gamma)=7$ [Go1], and $M=M_{5}$ or $M_{10}$ in [GW], with $\Delta(\beta, \gamma)=5$. In fact the only examples with $\Delta(\beta, \gamma)=5$ where $M(\beta)$ (say) contains a non-separating torus are $M_{5}, M_{10}$ and $M_{11}$ (see [GW, Lemma 23.1]). Now in [MP] three examples of hyperbolic knot manifolds are given, each with a pair of toroidal fillings at distance 5 , one of which contains a non-separating torus: these are $W h(-7 / 2), W h(-4 / 3)$ and $N(-5,5)$, described in Tables A.3, A.4 and A.9, respectively. By comparing 
the description in these tables of the second toroidal filling at distance 5 with that given in [GW, Lemma 22.2], we see that $W h(-7 / 2)=M_{10}, N(-5,5)=M_{11}$, and (hence) $W h(-4 / 3)=M_{5}$. It is well known that $W h(\delta)$ contains a once-punctured essential torus of slope 0 . The determination of the slopes $\gamma, \beta$ as listed in parts $(3)(b)$ and (3)(c) has been done by Martelli and Petronio. See [MP Tables A.2 and A.3].

Proof of Theorem 1.5. Let $K \subset S^{3}$ be a hyperbolic knot of genus one with exterior $M_{K}$ and suppose $p / q$ is an exceptional filling slope on $\partial M_{K}$ where $q \geq 1$.

Hyperbolic genus one knots in the 3 -sphere do not admit reducible surgery slopes [BZ1, so an exceptional surgery slope is either toroidal or irreducible, atoroidal, small Seifert. If $K$ is fibred, it is necessarily the figure eight knot, and the theorem holds in this case. Assume that $K$ is not a fibred knot. Then

(a) $M_{K}(0)$ is not fibred Ga,

(b) $K$ admits no $L$-space surgery $\mathrm{Ni}$,

(c) $K$ is not a Eudave-Muñoz knot [E-M].

A genus one Seifert surface for $K$ completes to an essential torus in $M_{K}(0)$ Ga. Suppose that $M_{K}(0)$ is Seifert fibred. As its first homology group is infinite cyclic, its base orbifold must have underlying space $S^{2}$ and $M_{K}(0)$ must have a non-zero Euler number. Thus it admits a non-separating, horizontal surface, which implies $M_{K}(0)$ fibres over the circle, contrary to (a). Thus $M_{K}(0)$ is not Seifert fibred, so assertion (1) of the theorem holds.

By (b), $K$ has no finite surgery slopes. Thus if $M_{K}(p / q)$ is small Seifert with base orbifold $S^{2}(a, b, c)$, then $p \neq 0$ and $(a, b, c)$ is either a Euclidean or hyperbolic triple, so $|p| \leq 3$ by Theorem 1.3 . Consideration of $H_{1}\left(S^{2}(a, b, c)\right)$ shows that $(a, b, c)$ is a hyperbolic triple. Hence assertion (2) of the theorem holds.

Theorem 1.3 combines with (b) and assertion (2) to show that if $M_{K}(p / q)$ is small Seifert then $0<|p| \leq 3$. Thus assertion (3) of the theorem holds.

Since $K$ is not a Eudave-Muñoz knot, each toroidal slope of $K$ is integral. It follows from [Go1] and [Te1] that no genus one knot in the 3-sphere admits a toroidal filling slope of distance 5 or more from the longitude. Such knots with toroidal slopes of distance 4 are determined in GW, Theorem 24.4]. In particular, all such knots are twist knots and the non-longitudinal slope is \pm 4 . This proves assertion (4).

\section{REFERENCES}

[Ba] K. Baker, Once-punctured tori and knots in lens spaces, Comm. Anal. Geom. 19 (2011), 347-399. MR2835883

[BiMe] J. Birman and Wm. Menasco, Studying links via closed braids III, Pacific J. Math. 161 (1993), 25-113. MR.1237139 (94i:57005)

[BCSZ1] S. Boyer, M. Culler, P. Shalen, and X. Zhang, Characteristic submanifold theory and Dehn filling, Trans. Amer. Math. Soc. 357 (2005), 2389-2444. MR.2140444 (2006a:57018)

[BCSZ2] , Characteristic subvarieties, character varieties, and Dehn fillings, Geometry \& Topology 12 (2008) 233-297. MR2390346 (2009a:57003)

[BGZ1] S. Boyer, C. McA. Gordon and X. Zhang, Dehn fillings of large hyperbolic 3-manifolds, J. Diff. Geom. 58 (2001), 263-308. MR1913944 (2003j:57025)

[BGZ2] C Characteristic submanifold theory and toroidal Dehn filling, Adv. Math. 230 (2012), 1673-1737.

[BGZ3] , Dehn fillings of knot manifolds containing essential twice-punctured tori, in preparation. 
[BZ1] S. Boyer and X. Zhang, Reducing Dehn filling and toroidal Dehn filling, Topology Appl. 68 (1996) 285-303. MR1377050 (97f:57018)

[BZ2] _ _ On Culler-Shalen seminorms and Dehn fillings, Ann. Math. 148 (1998), 737801. MR1670053(2000d:57028)

[CGLS] M. Culler, C. M. Gordon, J. Luecke and P. Shalen, Dehn surgery on knots, Ann. of Math. 125 (1987) 237-300. MR881270 (88a:57026)

[CJR] M. Culler, W. Jaco and H. Rubinstein, Incompressible surfaces in once-punctured torus bundles, Proc. Lond. Math. Soc. 45 (1982) 385-419. MR675414 (84a:57010)

[Du] Wm. Dunbar, Geometric orbifolds, Rev. Mat. 1 (1988) 67-99. MR977042 (90k:22011)

[E-M] M. Eudave-Muñoz, Non-hyperbolic manifolds obtained by Dehn surgery on hyperbolic knots, Geometric topology (Athens, GA, 1993), 35-61, AMS/IP Stud. Adv. Math., 2.1, Amer. Math. Soc., Providence, RI, 1997. MR1470720 (98i:57007)

[FH] W. Floyd and A. Hatcher, Incompressible surfaces in punctured-torus bundles, Top. Appl. 13 (1982), 263-282. MR651509 (83h:57015)

[FKP] D. Futer, E. Kalfagianni, and J. Purcell, Cusp areas of Farey manifolds and applications to knot theory, Int. Math. Res. Not. 2010, no. 23, 4434-4497. MR2739802 (2011k:57027)

[Ga] D. Gabai, Foliations and the topology of 3-manifolds II, J. Diff. Geom. 26 (1987), 461478. MR910017 (89a:57014a)

[Go1] C. McA. Gordon, Boundary slopes of punctured tori in 3-manifolds, Trans. Amer. Math. Soc. 350 (1998) 1713-1790. MR.1390037 (98h:57032)

[Go2] _ Dehn filling: a survey, in Knot Theory, Banach Center Publications 42, Institute of Mathematics, Polish Academy of Sciences, Warsaw, 1998, 129-144. MR 1634453 (99e:57028)

[GLi] C. McA. Gordon and R. A. Litherland, Incompressible surfaces in branched coverings, The Smith conjecture, Pure Appl. Math., 112, Academic Press (1984) 139-152. MR758466

[GL] C. McA. Gordon and J. Luecke, Toroidal and boundary-reducing Dehn Fillings, Topology Appl. 93 (1999) 77-90. MR.1684214(2000b:57030)

[GW] C. McA. Gordon and Y.-Q. Wu, Toroidal Dehn fillings on hyperbolic 3-manifolds, Mem. Amer. Math. Soc. 194 (2008), no.909. MR2419168 (2009c:57036)

[GW2] _ Toroidal and annular Dehn fillings, Proc. London Math. Soc. 78 (1999) 662700. MR,1674841 (2000b:57029)

[HR] C. Hodgson and H. Rubinstein, Involutions and isotopies of lens spaces, in Knot Theory and Manifolds, ed. D. Rolfsen, Lecture Notes in Mathematics 1144, Springer-Verlag, 1983, 60-96. MR 823282(87h:57028)

[KT] P. K. Kim and J. L. Tollefson, Splitting the PL involutions of nonprime 3-manifolds, Michigan Math. J. 27 (1980) 259-274. MR584691 (81m:57007)

[LM] M. Lackenby and R. Meyerhoff, The maximal number of exceptional Dehn surgeries, preprint (2008), arXiv:0808.1176.

[L1] S. Lee, Dehn fillings yielding Klein bottles, Int. Math. Res. Not. 2006, Art. ID 24253, 34pp. MR2219228(2007b:57038)

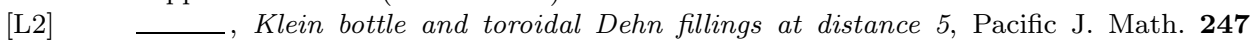
(2010), 407-434. MR2734156 (2012e:57036)

[L3] Lens spaces and toroidal Dehn fillings, Math. Z. 267 (2011), 781-802. MR2776058 (2012a:57022)

[LT] S. Lee and M. Teragaito, Boundary structure of hyperbolic 3-manifolds admitting annular and toroidal fillings at large distance, Canad. J. Math. 60 (2008) 164-188. MR2381171 (2009a:57029)

[MP] B. Martelli and C. Petronio, Dehn filling of the "magic" 3-manifold, Comm. Anal. Geom. 14 (2006), 969-1026. MR 2287152(2007k:57042)

[MSch] Y. Moriah and J. Schultens, Irreducible Heegaard splittings of Seifert fibred spaces are either vertical or horizontal, Topology 37 (1998) 1089-1112. MR.1650355 (99g:57021)

[Ni] Y. Ni, Knot Floer homology detects fibred knots, Invent. Math. 170 (2007), no. 3, 577608. MR2357503 (2008j:57053)

[Oe] U. Oertel, Closed incompressible surfaces in complements of star links, Pacific J. Math. 111 (1984), 209-230. MR732067 (85j:57008)

[Oh] S. Oh, Reducible and toroidal manifolds obtained by Dehn filling, Top. Appl. 75 (1997), 93-104. MR1425387(98a:57027) 
[Sh] H. Short, Some closed incompressible surfaces in knot complements which survive surgery, in Low dimensional topology, ed. Roger Fenn, London Math. Soc. Lecture Notes 95, Cambridge University Press, 1985, 179-194. MR827302 (88d:57006)

[Te1] M. Teragaito, Distance between toroidal surgeries on hyperbolic knots in the 3-sphere, Trans. Amer. Math. Soc. 358 (2006), 1051-1075. MR2187645 (2006h:57005)

[Te2] Creating Klein bottles by surgery on knots, J. Knot Theory Ramifications 10 (2001) 781-794. MR.1839702 (2002f:57017)

[Wu1] Y.-Q. Wu, Incompressibility of surfaces in surgered 3-manifolds, Topology, 31 (1992) 271-279. MR1167169 (94e:57027)

[Wu2] _ Dehn fillings producing reducible manifolds and toroidal manifolds, Topology 37 (1998), 95-108. MR 1480879 (98j:57033)

[YM] S-T Yau and W. Meeks, The equivariant loop theorem for three-dimensional manifolds and a review of the existence theorem for minimal surfaces, in The Smith Conjecture, Pure Appl. Math., 112, Academic Press (1984) 153-163. MR758467

Département de Mathématiques, Université du Québec à Montréal, 201 avenue du Président-Kennedy, Montréal, QuéBec, Canada H2X 3 Y7

E-mail address: boyer.steven@uqam.ca

$U R L:$ http://www.cirget.uqam.ca/boyer/boyer.html

Department of Mathematics, University of Texas at Austin, 1 University Station, Austin, Texas 78712

E-mail address: gordon@math.utexas.edu

URL: http://www.ma.utexas.edu/text/webpages/gordon.html

Department of Mathematics, SUNy at Buffalo, Buffalo, New York 14214-3093

E-mail address: xinzhang@buffalo.edu

URL: http: //www. math.buffalo.edu/ ${ }^{\sim}$ xinzhang 